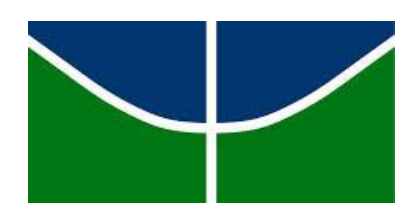

UNIVERSIDADE DE BRASÍLIA

INSTITUTO DE CIÊNCIAS EXATAS

DEPARTAMENTO DE MATEMÁTICA

Conjectura de Hanna Neumann: das origens à demonstração

por

Gláucia Lenita Dierings

Brasília

2016 
Gláucia Lenita Dierings

\section{Conjectura de Hanna Neumann: das origens à demonstração}

Dissertação apresentada ao Programa de PósGraduação em Matemática da Universidade de Brasília, como requisito parcial para obtenção do título de Mestre em Matemática.

Orientadora: Profa. Dra. Sheila Campos Chagas.

Brasília

2016 
Ficha catalográfica elaborada automaticamente, com os dados fornecidos pelo(a) autor(a)

Dierings, Gláucia Lenita

DD559c Conjectura de Hanna Neumann: das origens à demonstração / Gláucia Lenita Dierings; orientador Sheila Campos Chagas. -- Brasília, 2016. $85 \mathrm{p}$.

Dissertação (Mestrado - Mestrado em Matemática) -Universidade de Brasília, 2016.

1. Conjectura de Hanna Neumann. 2. Grupos Livres. I. Campos Chagas, Sheila, orient. II. Título. 
Universidade de Brasília

Instituto de Ciências Exatas

Departamento de Matemática

\title{
Conjectura de Hanna Neumann: das origens à
}

\section{demonstração}

\author{
por \\ GLÁUCIA LENITA DIERINGS *
}

Dissertação apresentada ao Departamento de Matemática da Universidade

de Brasília, como parte dos requisitos para obtenção do grau de

\section{MESTRE EM MATEMÁTIC̣A}

Brasília, 24 de fevereiro de 2016.

Comissão Examinadora:

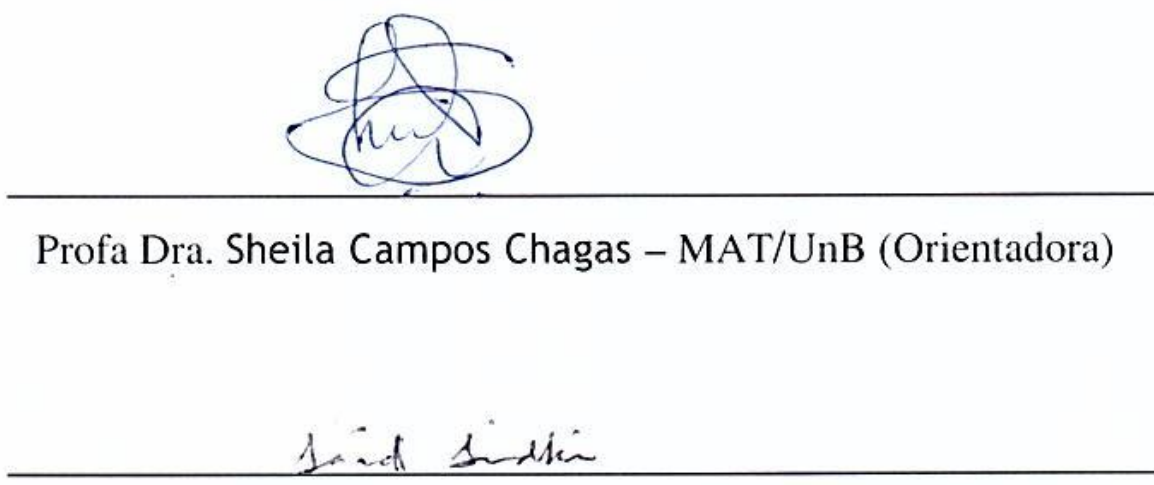

Prof. Dr. Prof. Said Najati Sidki - MAT/UnB (Membro)

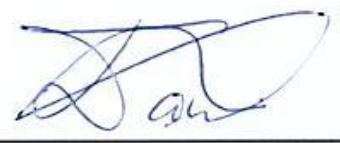

Profa. Dra.. Dessislava Hristova Kochloukova - UNICAMP

* O autor foi bolsista do CNPq durante a elaboração desta dissertação. 


\section{Agradecimentos}

Primeiramente, agradeço a Deus por guiar meus passos, me proteger e abençoar minha vida com sua sabedoria.

Agradeço à minha família, pai João Dierings, mãe Celi Maria Zimmer Dierings e irmã Milena Beatriz Dierings por todo apoio e carinho nessa caminhada. Mesmo distantes, foram minha base e inspiração, sem o amor de vocês não teria chegado até aqui.

Agradeço aos meus amigos pelo apoio e amizade, o que foi fundamental nessa jornada. Em primeiro lugar, à minha grande amiga irmã Alessandra Kreutz que aceitou esse desafio comigo e me acompanhou sempre. Obrigada por todos os conselhos, estudos, incentivos e bons momentos vividos. Agradeço aos grandes amigos feitos em Brasília/DF, os quais considero minha segunda família e tornam meus dias aqui mais felizes: Débora Heinen Kist, Jaqueline Thiele, Sônia da Costa, Vicente Puhl e família. Agradeço ao meu amigo e colega algebrista Michell L. Dias por todo incentivo e estudos juntos. Um agradecimento especial às minhas queridas amigas de Santa Maria/RS, que mesmo estando longe sempre torceram por mim: Débora Dalmolin, Alesandra T. Ximendes, Bruna Pavlack, Edinéia Filipiak, Jaqueline e Janaíne Welter.

Agradeço à professora Sheila Campos Chagas pela orientação e por todos os conhecimentos que juntas construímos. Foi uma experiência muito válida. Agradeço também aos demais professores que contribuíram para minha formação ao longo de toda minha vida acadêmica.

Agradeço aos professores Sheila Campos Chagas, Dessislava Hristova Kochloukova e Said Najati Sidki pela disponibilidade de compor a banca avaliadora, pelos conselhos e elogios.

Por fim, agradeço ao CNPp pelo apoio financeiro na realização desta pesquisa. 


\section{Resumo}

O objetivo desse trabalho é estudar a Conjectura de Hanna Neumann, que surgiu a partir do conhecido resultado provado por Howson e fornece um limitante para o posto da interseção de dois subgrupos finitamente gerados de um grupo livre. Serão apresentados alguns resultados intermediários desde 1957, ano em que a conjectura foi proposta, até sua demonstração em 2011. Apresentaremos a demonstração feita por Igor Mineyev em [18] e a versão de Warren Dicks, a qual simplificou a primeira prova dada por Mineyev em [17]. Além disso, será apresentado um resultado que garante que o limite proposto por Hanna Neumann é o melhor possível.

Palavras-chave: Conjectura de Hanna Neumann. Grupos livres. 


\section{Abstract}

The purpose of this work is studying the Hanna Neumann Conjecture, that came from the theorem proved by Howson and gives a upper bound for the rank of the intersection of two finitely generated subgroups of a free group. We shall present some intermediate results since 1957, year in which the conjecture was proposed, until be proved in 2011 . We shall give the proof presented by Igor Mineyev in [18] and the version of Warren Dicks that simplified the first Mineyev's proof in [17]. Moreover, we shall present a result that says the upper bound suggested by the Hanna Neumann conjecture cannot be improved. Keywords: Hanna Neumann Conjecture. Free groups. 


\section{Sumário}

$\begin{array}{ll}\text { Introdução } & 1\end{array}$

1 Grupos Livres 5

1.1 Caracterização dos grupos livres . . . . . . . . . . . . . . . . . . 5

1.2 O Teorema de Nielsen-Schreier . . . . . . . . . . . . . . . . . . . . 11

2 Grafos $\quad 17$

2.1 Morfismo de grafos . . . . . . . . . . . . . . . . . . 22

2.2 Ação de grupos em grafos . . . . . . . . . . . . . . . . . . . . . 24

2.3 Grupo fundamental de um grafo . . . . . . . . . . . . . . . . . 29

2.4 Recobrimento de grafos . . . . . . . . . . . . . . . . . 31

2.5 Grafos de grupos . . . . . . . . . . . . . . . . . . . 35

3 A Conjectura de Hanna Neumann 38

3.1 A Conjectura . . . . . . . . . . . . . . . . . . 38

3.2 Versão forte da Conjectura . . . . . . . . . . . . . . . . . 44

3.3 Demonstração da Conjectura . . . . . . . . . . . . . . . . . . . . . . 51

3.3.1 Construção de folhagens e sistemas . . . . . . . . . . . . . . . . 52

3.3.2 Ordenação e conjuntos ordem-essencial . . . . . . . . . . . . . 58

3.4 A demonstração de Warren Dicks . . . . . . . . . . . . . . . . 68

3.5 O limitante superior é $\operatorname{sharp} \ldots \ldots \ldots \ldots \ldots$. . . . . . . . . . . . . 75

$\begin{array}{ll}\text { Referências Bibliográficas } & 83\end{array}$ 


\section{Introdução}

O estudo dos grupos livres iniciou-se por volta de 1880 através do trabalho desenvolvido por Walther von Dick, que definiu grupos finitamente gerados e apresentação de grupos por geradores e relações definidoras. Os grupos livres possuem a apresentação mais simples possível, sendo dada por um conjunto de geradores e um conjunto vazio de relações. O objetivo de Walther von Dick ao definir os grupos livres era tornar as descrições das apresentações dos grupos mais claras.

Um dos principais resultados no estudo dos grupos livres afirma que todo subgrupo de um grupo livre é livre. Esse teorema foi primeiramente provado por Jakob Nielsen, em 1921, para o caso em que o subgrupo é finitamente gerado. Em 1926, Otto Schreier retirou a hipótese da finitude e então o teorema ficou conhecido como Teorema de NielsenSchreier. No caso do subgrupo possuir índice finito, temos uma fórmula para o cálculo do posto desse subgrupo. O teorema pode ser assim enunciado:

Teorema 0.1 (Nielsen-Schreier). Seja F um grupo livre e $H$ um subgrupo de F, então $H$ é livre. Além disso, se $|F: H|=g$ e $\operatorname{rank}(F)=r$ são ambos finitos, $\operatorname{rank}(H)=(r-1) g+1$.

Note que o posto do subgrupo pode exceder o posto do grupo livre, mas, em alguns casos, não é fácil calculá-lo. Agora, dados dois subgrupos de um grupo livre, sua interseção será um um grupo livre e o cálculo do posto dessa interseção já se torna mais trabalhoso. Nesse sentido, entre os anos de 1954 e 1957, os trabalhos desenvolvidos por A. G. Howson e Hanna Neumann apresentaram resultados interessantes a respeito do posto da interseção de dois subgrupos finitamente gerados de um grupo livre.

Para descrever esses resultados, vamos considerar $F$ um grupo livre e $A$ e $B$ dois subgrupos finitamente gerados de $F$. No ano de 1954, Howson mostrou, em [11], que a interseção de $A$ e $B$ é finitamente gerada e encontrou um limitante para o posto dessa 
interseção: denotando os postos de $A, B$ e $A \cap B$ por $m, n$ e $\mathcal{N}$, respectivamente, Howson obteve que $\mathcal{N}-1 \leq 2 m n-m-n$.

Em 1956, Hanna Neumann em [20], apresentou uma pequena modificação da demonstração de Howson e mostrou que $\mathcal{N}-1 \leq 2 m n-2 m-n$, onde $m \geq n$. No ano seguinte, Hanna Neumann publicou um adendo (veja em [21]) em que melhorou o limite para $\mathcal{N}-1 \leq 2(m-1)(n-1)$ e fez a seguinte conjectura:

Conjectura de Hanna Neumann. Sejam A e B subgrupos finitamente gerados de um grupo livre $F$, de postos $m$ e $n$ respectivamente, e seja $\mathcal{N}$ o posto da interseção $A \cap B$. Então, $\mathcal{N}-1 \leq(m-1)(n-1)$.

A partir de então, vários matemáticos dedicaram-se a provar a Conjectura de Hanna Neumann. Diversos resultados foram obtidos nesta direção, mas somente em 2011 a conjectura foi provada independentemente por Joel Friedman e Igor Mineyev.

No ano de 1971, Burns, assumindo as mesmas condições de Hanna Neumann em seu trabalho, provou em [3] que $\mathcal{N}-1 \leq 2(m-1)(n-1)-\min (m-1, n-1)$, sendo o melhor limite provado até então. Walter Neumann, em 1990 (veja em [22]), formulou uma nova versão da conjectura, na qual em vez de considerar a interseção $A \cap B$, considerou um conjunto de representantes de classes de conjugação de interseções não triviais $y^{-1} A y \cap$ $z^{-1} B z$ e mostrou que o limitante para esse caso continua o mesmo. Essa versão ficou conhecida como a versão forte da conjectura e será descrita no Capítulo 3.

A partir do artigo publicado por Walter, todos os resultados subsequentes objetivaram provar a versão forte da conjectura. No ano de 1992, Tardos provou, em [27], a versão forte da conjectura para o caso onde pelo menos um dos subgrupos possui posto dois, sendo que analisou os subgrupos dos grupos livres e suas interseções usando grafos de subgrupos, técnica desenvolvida por Stallings em [25].

Em 1994, Warren Dicks (veja em [5]) mostrou que existe uma equivalência entre o limite superior proposto pela versão forte da conjectura e o melhor limite superior possível para o número de arestas de um grafo bipartido com certas condições de simetria, conhecida como a conjectura dos grafos amalgamados. Dois anos depois, Tardos em [28], demonstrou uma versão diferente do limite de Burns, o que provou a conjectura para o caso em que ambos os subgrupos possuem posto três. 
Em 2001, Dicks e Formanek, em [7], provaram a versão forte da conjectura para o caso em que pelo menos um dos subgrupos possui posto no máximo três. No ano seguinte, Meakin e Weil em [16] e, independentemente, Khan em [13], demonstraram a versão forte da conjectura para o caso em que um dos subgrupos é gerado positivamente, ou seja, por um conjunto finito que consiste apenas de palavras positivas.

Além dos trabalhos citados, outros matemáticos estudaram a interseção de grupos livres buscando demonstrar a conjectura, tais como S. V. Ivanov, P. Nickolas, W. Imrich, S.M. Gersten, T. Jitsukawa, A. G. Myasnikov, entre outros.

Em maio de 2011, Igor Mineyev e Joel Friedman demonstraram, em trabalhos independentes, que a conjectura é verdadeira. A demonstração dada por Joel Friedman pode ser encontrada em [10] e não será abordada nesse trabalho. A primeira versão apresentada por Mineyev, em [17], envolvia Módulos de Hilbert e um pouco de teoria de grafos. Após ler o trabalho de Mineyev, Warren Dicks apresentou uma simplificação usando a teoria de Bass-Serre (veja em [8]). Assim, com a contribuição de Dicks, Mineyev em [18] apresentou uma segunda versão da demonstração com o artigo intitulado Groups, graphs, and the Hanna Neumann Conjecture, a qual utiliza basicamente a teoria de grupos e de grafos, e que será aqui apresentada.

O objetivo desse trabalho é apresentar alguns dos principais resultados obtidos ao longo dos mais de cinquenta anos em que buscou-se provar a conjectura, culminando com a demontração da mesma. Para isso, apresentaremos inicialmente resultados necessários de teoria dos grupos livres e de teoria de grafos, baseado nas referências [2], [4], [6], [12] e $[25]$.

No Capítulo 1, apresentaremos a definição de grupo livre e suas principais caracterizações. Além disso, provaremos o Teorema de Nielsen-Schreier, encontrando a fórmula para o posto do subgrupo para o caso em que o índice do subgrupo e posto do grupo livre são finitos. No Capítulo 2, desenvolveremos a teoria de grafos, apresentando definições importantes, tais como morfismo, recobrimento de grafos e grafos de grupos. Além disso, apresentaremos exemplos e resultados necessários para as demonstrações que serão dadas na sequência.

O Capítulo 3, intitulado Conjectura de Hanna Neumann, apresentará inicialmente o resultado original demonstrado por Hanna Neumann e baseado no trabalho de Howson. 
Na sequência, definiremos a versão forte da conjectura proposta por Walter Neumann, mostrando que o limitante para esse caso continua o mesmo. Depois, demonstraremos a conjectura a partir do trabalho desenvolvido por Mineyev, utilizando como ferramentas a construção de folhagens e sistemas, a ordenação dos grafos com os quais trabalharemos e a definição de conjuntos ordem-essencial.

Além disso, apresentaremos a demonstração da conjectura dada por Dicks, a qual foi fundamental para o segundo trabalho publicado de Mineyev. Finalizaremos com um teorema proposto por Richard Kent em [14], no ano de 2005, que garante que o limite superior sugerido pela Conjectura de Hanna Neumann é o melhor possível. 


\section{Capítulo 1}

\section{Grupos Livres}

Neste capítulo, introduziremos a definição de grupo livre e apresentaremos algumas características importantes dos mesmos. Um dos principais resultados será o teorema de Nielsen-Schreier, que demonstra que todo subgrupo de um grupo livre é livre.

\subsection{Caracterização dos grupos livres}

Definição 1.1. Seja $X$ um subconjunto de um grupo $F$, então $F$ é dito um grupo livre sobre $X$ se para todo grupo $G$ e toda função $f: X \rightarrow G$ existe um único homomorfismo $\varphi: F \rightarrow G$ tal que $\varphi(x)=f(x)$, para todo $x \in X$.

Em outras palavras, podemos dizer que o diagrama abaixo comuta:

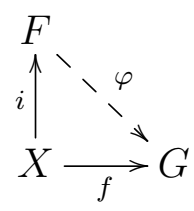

Uma segunda definição que podemos dar, é que $F$ é um grupo livre sobre $X$ se todo elemento não trivial $f \in F$ pode ser escrito unicamente como produto $f=x_{1} x_{2} \ldots x_{n}$, onde $x_{i} \in X \cup X^{-1}$ e $x_{i} x_{i+1} \neq 1$.

Agora, precisamos construir os grupos livres para garantirmos sua existência. Para isso, vamos pensar nos elementos do grupo livre como palavras reduzidas no conjunto $X$. Considere $X$ um conjunto não vazio e $X^{-1}$ uma cópia disjunta de $X$. Assim, existe uma bijeção $\pi: X \rightarrow X^{-1}$. Definimos um alfabeto sobre $X$ como sendo $X \cup X^{-1}$. 
Definição 1.2. Uma palavra sobre $X$ de comprimento $n, n \geq 1, n \in \mathbb{Z}$, é uma função $\omega:\{1,2, \ldots, n\} \rightarrow X \cup X^{-1}$. Ou seja, se $\omega(i)=x_{i}^{e_{i}}$, então uma palavra de comprimento $n$ será dada por $\omega=x_{1}^{e_{1}} x_{2}^{e_{2}} \ldots x_{n}^{e_{n}}, x_{i} \in X$ e $e_{i}= \pm 1$.

Exemplo 1.3. Se $X=\{a, b, c\}$ então $X^{-1}=\left\{a^{-1}, b^{-1}, c^{-1}\right\}$ e $a b c^{-1}, a b b^{-1} a^{-1} c$ são exemplos de palavras sobre $X$. Se $\omega=a a^{-1}$, temos que o comprimento de $\omega$ será $|\omega|=2$.

Definimos abaixo algumas características importantes das palavras:

Definição 1.4. $\quad$ 1. A palavra vazia é denotada por 1 e tem comprimento zero;

2. Uma subpalavra de $\omega=x_{1}^{e_{1}} x_{2}^{e_{2}} \ldots x_{n}^{e_{n}}$ ou é a palavra vazia, ou é da forma $\omega=$ $x_{r}^{e_{r}} \ldots x_{s}^{e_{s}}$, onde $1 \leq r, s \leq n$;

3. O inverso de uma palavra $\omega=x_{1}^{e_{1}} x_{2}^{e_{2}} \ldots x_{n}^{e_{n}}$ é $\omega^{-1}=x_{n}^{-e_{n}} \ldots x_{1}^{-e_{1}}$;

4. Uma palavra $\omega$ é reduzida se $w=1$ ou se $\omega$ não tem subpalavra da forma $x x^{-1}$ ou $x^{-1} x$, onde $x \in X$;

5. Se $u=x_{1}^{e_{1}} x_{2}^{e_{2}} \ldots x_{n}^{e_{n}}$ e $v=y_{1}^{d_{1}} y_{2}^{d_{2}} \ldots y_{m}^{d_{m}}$ são palavras em $X$, então sua justaposição será dada pela palavra $u v=x_{1}^{e_{1}} \ldots x_{n}^{e_{n}} y_{1}^{d_{1}} \ldots y_{m}^{d_{m}}$

Vamos definir grupo livre como o conjunto de todas as palavras sobre $X$ com a operação de justaposição, com a identidade sendo a palavra vazia e com o inverso da palavra $\omega$ sendo $\omega^{-1}$. Mas para isso, precisamos restringir os elementos do grupo livre somente ao conjunto das palavras reduzidas sobre $X$. O processo de redução descrito a seguir nos permite obter uma palavra reduzida de uma palavra arbitrária.

Definição 1.5. Sejam $A$ e $B$ palavras sobre $X$ e $\omega=A B$, então as operações elementares são:

Inserção: troca $\omega=A B$ por $\omega=A x x^{-1} B$, para $x \in X \cup X^{-1}$.

Deletação: troca $\omega=A x x^{-1} B$ para $\omega=A B$, para $x \in X \cup X^{-1}$.

Então, dadas duas palavras $\omega$ e $\omega^{\prime}$, escreveremos $\omega \rightarrow \omega^{\prime}$ para dizer que $\omega^{\prime}$ decorre de $\omega$ por uma operação elementar. 
Definição 1.6. Duas palavras u e $v$ sobre $X$ são equivalentes, e escrevemos $u \sim v$, se existem palavras $u=\omega_{1}, \omega_{2}, \ldots, \omega_{n}=v$ e operações elementares $u=\omega_{1} \rightarrow \omega_{2} \rightarrow \cdots \rightarrow$ $\omega_{n}=v$.

Exemplo 1.7. A palavra equivalente reduzida da palavra $a^{-1} b b^{-1} a c d c^{-1} c c c^{-1} d^{-1} c^{-1} d a^{-1}$ será a palavra $\mathrm{da}^{-1}$ :

$$
a^{-1} b b^{-1} a c d c^{-1} c c c^{-1} d^{-1} c^{-1} d a^{-1} \rightarrow a^{-1} a c d d^{-1} c^{-1} d a^{-1} \rightarrow c c^{-1} d a^{-1} \rightarrow d a^{-1}
$$

Seja $W$ o conjunto de todas as palavras no alfabeto $X \cup X^{-1}$. Vamos denotar por $F$ o conjunto de classes de equivalência das palavras de $W$ e por $[\omega]$ a classe de equivalência de uma palavra $\omega$.

Proposição 1.8. Qualquer classe de equivalência $[\omega]$ contém uma única palavra reduzida.

Demonstração. Primeiro vamos provar a existência da palavra reduzida. Se $\omega$ não possui subpalavra da forma $x x^{-1}$, com $x \in X \cup X^{-1}$, então $\omega$ é reduzida. Se $\omega$ possui, deleta-se a subpalavra $x x^{-1}$, obtendo uma nova palavra $\omega_{1}$. Se $\omega_{1}$ é reduzida terminou, senão existe uma subpalavra de $\omega_{1}$ da forma $x x^{-1}$ e deletando-a obtém-se uma nova palavra $\omega_{2}$. Como os comprimentos das palavras são estritamente decrescentes, o processo termina com uma palavra reduzida equivalente a $\omega$.

Para provar a unicidade, suponha que existam duas palavras reduzidas distintas $u$ e $v$ em $[\omega]$ e escolha a cadeia de operações elementares $u=\omega_{1} \rightarrow \omega_{2} \rightarrow \cdots \rightarrow \omega_{k}=v$ tal que $\sum_{i=1}^{k}\left|\omega_{i}\right|$ é mínimo. Já que as palavras $u$ e $v$ são reduzidas e distintas, teremos $\left|\omega_{1}\right|<\left|\omega_{2}\right|$, pois faremos uma inserção de $\omega_{1}$ para $\omega_{2}$ e $\left|\omega_{k-1}\right|>\left|\omega_{k}\right|$, pois de $\omega_{k-1}$ para $\omega_{k}$ faremos uma deletação. Portanto, existe $i \in\{2, \ldots, k-1\}$ tal que $\left|\omega_{i-1}\right|<\left|\omega_{i}\right|$ e $\left|\omega_{i}\right|>\left|\omega_{i+1}\right|$. Então $\omega_{i+1}$ pode ser obtida de $\omega_{i-1}$ primeiro inserindo a subpalavra $x x^{-1}$ e depois deletando uma subpalavra $y y^{-1}$. Se essas subpalavras são disjuntas em $\omega_{i}$, podemos primeiro deletar $y y^{-1}$, obtendo uma nova palavra $\omega_{i}^{\prime}$, e depois inserir $x x^{-1}$. Assim, podemos substituir $\omega_{i-1} \rightarrow \omega_{i} \rightarrow \omega_{i+1}$ por $\omega_{i-1} \rightarrow \omega_{i}^{\prime} \rightarrow \omega_{i+1}$ e obtemos uma nova cadeia conectando $u$ e $v$ com menor soma de comprimentos, uma contradição. No caso das palavras $x x^{-1}$ e $y y^{-1}$ de $\omega_{i}$ possuírem interseção não vazia, $\omega_{i-1}=\omega_{i+1}$ e podemos deletar as palavras $\omega_{i}$ e $\omega_{i-1}$ da sequência e novamente chegamos a uma contradição com 
a minimalidade da soma. Logo não existem duas palavras reduzidas distintas na mesma classe de equivalência.

Podemos provar agora a existência de um grupo livre com base $X$. Esse grupo livre será o o grupo $F$ com a operação de justaposição.

Proposição 1.9. Para todo conjunto $X$ existe um grupo livre com base $X$.

Demonstração. Dadas duas palavras $u$ e $v$ em $W$, defina a multiplicação no conjunto $F$ por $[u][v]=[u v]$. Vamos provar que $F$ é um grupo livre com base $[X]=\{[x] \mid x \in X\}$. A associatividade da multiplicação é válida, pois dados $u, v, w \in W$, temos

$$
[u]([v][w])=[u][v w]=[u(v w)]=[(u v) w]=[u v][w]=([u][v])[w]
$$

A identidade é a classe [1] e o inverso da classe $[\omega]=\left[x_{1}^{e_{1}} x_{2}^{e_{2}} \ldots x_{n}^{e_{n}}\right]$ é a classe $\left[x_{n}^{-e_{n}} \ldots x_{1}^{-e_{1}}\right]$. Portanto, $F$ é um grupo. Além disso, $[\omega]=\left[x_{1}^{e_{1}}\right]\left[x_{2}^{e_{2}}\right] \ldots\left[x_{n}^{e_{n}}\right]$ e essa expressão é reduzida com respeito a $X$ se, e somente se, a palavra $\omega$ é reduzida. A unicidade da forma reduzida dos elementos de $F$ com respeito a $[X]$ segue do fato provado anteriormente, de que cada classe contém exatamente uma palavra reduzida. E como a cardinalidade do conjunto $[X]$ é igual a cardinalidade de $X$, pela segunda definição de grupo livre, segue que $F$ é um grupo livre com base $[X]$.

Provada a existência de um grupo livre, podemos nos questionar se dois grupos livres com a mesma base são isomorfos ou se dois grupos livres isomorfos possuem a mesma base. Neste sentido, estamos interessados na unicidade dos grupos livres. Provaremos uma condição necessária e suficiente para que ela ocorra, mas antes considere a seguinte observação:

Observação 1.10. Sejam $F$ e $G$ grupos quaisquer $e X \subseteq F$. $\operatorname{Sejam} \operatorname{Hom}(F, G)$ o conjunto de todos os homomorfismos de $F$ em $G$ e $\mathcal{F}(X, G)$ o conjunto de todas as funções de $X$ em $G$. Então, considere

$$
\begin{aligned}
\theta: \operatorname{Hom}(F, G) & \longrightarrow \mathcal{F}(X, G) \\
(F \stackrel{\varphi}{\rightarrow} G) & \longmapsto(X \stackrel{i}{\rightarrow} F \stackrel{\varphi}{\rightarrow} G)
\end{aligned}
$$

Podemos dizer que $\theta$ é a função restrição $\varphi \circ i=\left.\varphi\right|_{X}$, ou seja, $\theta$ pega um homomorfismo $\varphi: F \rightarrow G$ e aplica na restrição à $X$. Então temos: 


$$
\begin{aligned}
\theta \text { é sobrejetiva } & \Longleftrightarrow \forall f \in \mathcal{F}(X, G), \exists \varphi \in \operatorname{Hom}(F, G) ; \varphi \circ i=f ; \\
\theta \text { é injetiva } & \Longleftrightarrow \text { se existe } \varphi \in \operatorname{Hom}(F, G) \text {, é único. }
\end{aligned}
$$

Logo, $F$ é um grupo livre sobre $X$ se, e somente se, $\theta$ é uma bijeção para todo grupo $G$.

Teorema 1.11. Sejam $F_{1}$ e $F_{2}$ dois grupos livres com bases $X_{1}$ e $X_{2}$, respectivamente. Então $F_{1} \cong F_{2}$ se, e somente se, $\left|X_{1}\right|=\left|X_{2}\right|$.

Demonstração. Suponha $F_{1} \cong F_{2}$. Na observação anterior, tomando $G=\mathbb{Z}_{2}$, considere as aplicações:

$$
\begin{aligned}
& \theta_{1}: \operatorname{Hom}\left(F_{1}, \mathbb{Z}_{2}\right) \longrightarrow \mathcal{F}\left(X_{1}, \mathbb{Z}_{2}\right) \\
&\left(F_{1} \stackrel{\varphi}{\rightarrow} \mathbb{Z}_{2}\right) \longmapsto\left(X_{1} \stackrel{i_{1}}{\rightarrow} F_{1} \stackrel{\varphi}{\rightarrow} \mathbb{Z}_{2}\right) \\
& \theta_{2}: \operatorname{Hom}\left(F_{2}, \mathbb{Z}_{2}\right) \longrightarrow \mathcal{F}\left(X_{2}, \mathbb{Z}_{2}\right) \\
&\left(F_{2} \stackrel{\phi}{\rightarrow} \mathbb{Z}_{2}\right) \longmapsto \\
&
\end{aligned}
$$

Agora, como $F_{1}$ é livre sobre $X_{1}$ e $F_{2}$ é livre sobre $X_{2}$, pela observação anterior, $\theta_{1}$ e $\theta_{2}$ são bijeções. Como $F_{1} \cong F_{2}$, temos $\left|\operatorname{Hom}\left(F_{1}, \mathbb{Z}_{2}\right)\right|=\left|\operatorname{Hom}\left(F_{2}, \mathbb{Z}_{2}\right)\right|$ e, portanto, $\left|\mathcal{F}\left(X_{1}, \mathbb{Z}_{2}\right)\right|=\left|\mathcal{F}\left(X_{2}, \mathbb{Z}_{2}\right)\right|$. Mas, se temos conjuntos $A$ e $B$ quaisquer, de cardinalidades $a$ e $b$, respectivamente, então $|F(A, B)|=b^{a}$. Portanto, $2^{\left|X_{1}\right|}=2^{\left|X_{2}\right|}$ e, assim, $\left|X_{1}\right|=\left|X_{2}\right|$.

Por outro lado, supondo $\left|X_{1}\right|=\left|X_{2}\right|$, existe uma bijeção $k: X_{1} \rightarrow X_{2}$. Sejam $\varphi$ e $\psi$ as extensões dadas pela propriedade universal dos grupos livres, então temos os seguintes diagramas:
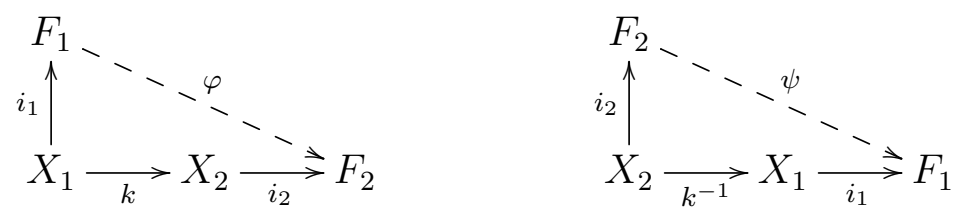

Note que para $x_{1} \in X_{1}$, temos $\psi \circ \varphi\left(x_{1}\right)=\psi\left(\varphi\left(x_{1}\right)\right)=\psi\left(k\left(x_{1}\right)\right)=k^{-1}\left(k\left(x_{1}\right)\right)=x_{1}$. Então, $\psi \circ \varphi: F_{1} \rightarrow F_{1}$ estende a inclusão $i_{1}: X_{1} \rightarrow F_{1}$, mas $1_{F_{1}}$ também estende. Logo, pela unicidade da extensão, segue que $\psi \circ \varphi=1_{F_{1}}$. Analogamente, obtemos que $\varphi \circ \psi=1_{F_{2}}$. Portanto, $\varphi$ é um isomorfismo e $F_{1} \cong F_{2}$. 
A partir desse teorema, podemos definir o que é o posto de um grupo livre:

Definição 1.12. O posto de um grupo livre $F$, denotado por rank $(F)$, é o número de elementos em sua base.

Assim, do Teorema 1.11, o posto de um grupo livre está bem definido, ou seja, independe da escolha da base de $F$. Note que se $G$ é um grupo qualquer, então o posto de $G$ é a cardinalidade do menor conjunto de geradores para $G$, isto é, o posto do menor grupo livre $F$ para o qual existe uma sobrejeção $\psi: F \rightarrow G$.

Não é difícil determinar o posto de um grupo livre, mas encontrar o posto de seus subgrupos é mais trabalhoso. A dificuldade aumenta quando consideramos a interseção de subgrupos do grupo livre e é nisso que estamos interessados.

Neste sentido, na próxima seção provaremos primeiro que todo subgrupo de um grupo livre também é livre, mas antes apresentamos uma caracterização interessante dos grupos livres.

Proposição 1.13 (Caracterização de grupo livre). Um grupo F é livre sobre um conjunto $X$ se, e somente se,

i) $X$ gera $F$;

ii) Nenhuma palavra reduzida em $X \cup X^{-1}$ de comprimento positivo é igual a 1.

Demonstração. $(\Leftarrow)$ Seja $\varphi: F(X) \rightarrow F$ o homomorfismo estendendo a inclusão $f: X \rightarrow$ $F$. Note que pelo item $i$ ) temos $\varphi$ sobrejetora e pelo item $i i), \operatorname{Ker} \varphi=\{1\}, \log \varphi \varphi$ é injetora. Portanto, $F$ é livre sobre $X$.

$(\Rightarrow)$ Seja $F$ um grupo livre sobre $X$. Vamos primeiro mostrar $i$ ). Considere $H=$ $\langle X\rangle=\cap\{Y \leq F \mid Y \supseteq X\}$. Considere $f: X \rightarrow H$ e $j: H \rightarrow F$ inclusões. Como $F$ é grupo livre, existe um único homomorfismo $\varphi: F \rightarrow H$ tal que $\varphi \circ i=f$. Note que $j \circ \varphi: F \rightarrow F$ estende $i: X \rightarrow F$.

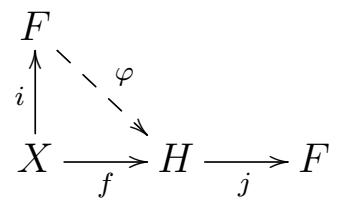


Mas $1_{F}$ também estende, logo pela unicidade temos $j \circ \varphi=1_{F}$. Portanto, $F=\operatorname{Im} 1_{F}=$ $\operatorname{Imj} \circ \varphi=\operatorname{Im} \varphi \subset H$. Logo, $F=H$. Para o item $i i)$, como $\varphi$ é injetiva, $\operatorname{Ker} \varphi=\{1\}$, logo não existe palavra reduzida em $X \cup X^{-1}$ de comprimento positivo igual a 1 .

\subsection{O Teorema de Nielsen-Schreier}

Agora, vamos provar um dos teoremas fundamentais da teoria de grupos livres, que diz que todo subgrupo de um grupo livre é livre. Este teorema foi primeiramente provado por Jakob Nielsen em 1921 para o caso em que o subgrupo é finitamente gerado. Em 1926, Otto Schreier retirou a hipótese da finitude e então o teorema ficou conhecido como Teorema de Nielsen-Schreier. Através desse teorema, veremos que o posto do subgrupo pode exceder o posto do grupo livre.

Utilizaremos o método de Schreier, que nos fornece um meio para encontrar uma apresentação para o subgrupo e fornece a fórmula para o posto do subgrupo, quando o índice do subgrupo e o posto do grupo livre são finitos.

Seja $F$ um grupo livre sobre $X$. Para a construção da demonstração, precisamos ordenar os elementos do grupo $F$. Então, primeiramente vamos tomar uma boa ordenação qualquer sobre o conjunto $X \cup X^{-1}$. Em seguida, ordenaremos os elementos de $F$ pelo seu comprimento e, por fim, aplicaremos a regra lexicográfica sobre os elementos de mesmo comprimento, como descreveremos a seguir.

Definição 1.14. Seja < uma boa ordenação sobre o conjunto $X \cup X^{-1}$ e sejam $a=$ $x_{1} x_{2} \ldots x_{n}$ e $b=y_{1} y_{2} \ldots y_{m}$, com $a \neq b$, palavras reduzidas em $F$. Então $a<b$ se $n<m$ ou se $n=m$ e $x_{r}<y_{r}$ em $X \cup X^{-1}$, onde $r=\min \left\{i \mid x_{i} \neq y_{i}\right\}$.

Exemplo 1.15. Seja $F$ grupo livre de posto 2 sobre $X=\{x, y\}$ e $X \cup X^{-1}$ ordenado como $x<y<x^{-1}<y^{-1}$. Então os 12 primeiros elementos terão a seguinte ordenação:

$$
1<x<y<x^{-1}<y^{-1}<x^{2}<x y<x y^{-1}<y x<y^{2}<y x^{-1}<x^{-1} y<\ldots
$$

Lema 1.16. Seja $\omega=x_{1} x_{2} \ldots x_{n-1} x_{n}$ uma palavra reduzida sobre $X \cup X^{-1}$ com $n>1 e$ seja $v \in F$ um elemento qualquer. Então $v<x_{1} x_{2} \ldots x_{n-1}$ implica que $v x_{n}<\omega$.

Demonstração. Se denotarmos por $l(v)$ o comprimento do elemento $v$, então $l(v)<n-1$ implica que $l\left(v x_{n}\right)<n-1+1=n=l(\omega)$. Logo $v x_{n}<\omega$. 
Podemos assumir que $v=x_{1} x_{2} \ldots x_{r-1} y_{r} \ldots y_{n-1}$ é uma palavra reduzida, onde $1 \leq$ $r \leq n-1$ e $y_{r}<x_{r}$. Se $y_{n-1}=x_{n}^{-1}$, então $l\left(v x_{n}\right)=n-2<l(\omega)$ e $v x_{n}<\omega$, senão $v x_{n}=x_{1} x_{2} \ldots x_{r-1} y_{r} \ldots y_{n-1} x_{n}$ está na forma reduzida, assim $v x_{n}<\omega$, pela definição de $<$.

Definição 1.17. Seja $H$ um subgrupo de $F$ e $H \omega$ uma classe lateral à direita de $H$ em F. Para cada escolha de um elemento em cada classe lateral à direita de $H$ em $F$ obtemos um transversal à direita $U$.

Vamos denotar a interseção $H \omega \cap U=\bar{\omega}$ para qualquer $\omega \in F$.

Definição 1.18. Um subconjunto não vazio $S$ de $F$ tem a propriedade de Schreier se contém todos os seus segmentos iniciais, isto é, se $\omega=x_{1} x_{2} \ldots x_{n} \in S$, então $x_{1} x_{2} \ldots x_{n-1} \in S$, onde $l(\omega)=n \geq 1$.

Um transversal de Schreier para $H$ em $F$ é um transversal à direita de $H$ em $F$ que tem a propriedade de Schreier.

Lema 1.19. Todo subgrupo $H$ de F tem um transversal de Schreier, por exemplo, o que é obtido escolhendo-se o menor elemento de cada classe lateral à direita de $H$ em F.

Demonstração. Seja $T$ o conjunto dos menores elementos em cada classe lateral à direita de $H$ em $F$. Vamos mostrar que $T$ satisfaz a propriedade de Schreier, isto é, se $\omega=$ $x_{1} \ldots x_{n} \in T$, então $x_{1} \ldots x_{n-1} \in T$.

Suponha que $x_{1} \ldots x_{n-1} \notin T$ e seja $v \in T$ o menor elemento da classe lateral $H x_{1} x_{2} \ldots$ $x_{n-1}$. Então temos que $v<x_{1} x_{2} \ldots x_{n-1}$ e pelo lema anterior, $v x_{n}<\omega$ e $H v=$ $H x_{1} \ldots x_{n-1}$ implica que $H v x_{n}=H \omega$. Então $v x_{n} \in H \omega$ e $v x_{n}<\omega$. Logo, $\omega$ não é o menor elemento de $H \omega$ e assim $\omega \notin T$, o que é uma contradição. Portanto, T satisfaz a propriedade de Schreier.

O objetivo agora é determinar os geradores de Schreier para um subgrupo $H$ do grupo livre $F$. Isto será feito em termos de um transversal de Schreier $U$ de $H$, dos geradores livres de $F$ e da função $F \longrightarrow U$ dada por $\omega \longmapsto \bar{\omega}$.

Note que $\bar{\omega}$ satisfaz $H \omega \cap U=\{\bar{\omega}\}$ e é o elemento de menor comprimento na classe $H \omega$. Além disso, temos $\overline{\bar{\omega}}=\bar{\omega}, H \omega=H \bar{\omega}, \forall \omega \in F$ e se $\omega \in U$, então $\bar{\omega}=\omega$. Observe 
também que, dado $u \in U$ e $x \in X \cup X^{-1}$ temos:

$$
H u x=H \overline{u x} \Rightarrow H u=H u x x^{-1}=H \overline{u x} x^{-1} \Rightarrow u=\overline{\overline{u x} x^{-1}}
$$

Lema 1.20. Os elementos do conjunto $A=\left\{u x \overline{u x}^{-1} \mid x \in X \cup X^{-1}\right\}$ geram $H$.

Demonstração. Note que como $H u x=H \overline{u x}$, temos $A \subset H$. Agora, seja $h \in H$ e escreva $h=x_{1} x_{2} \ldots x_{n}$, onde $x_{i} \in X \cup X^{-1}, 1 \leq i \leq n$, uma palavra reduzida em $X \cup X^{-1}$. Observe ainda que:

$$
h=x_{1} x_{2} \ldots x_{n}=1 x_{1}{\overline{1 x_{1}}}^{-1}{\overline{1 x_{1}}}_{2}{\overline{x_{1} x_{2}}}^{-1}{\overline{x_{1} x_{2}}}_{x_{3}} \ldots{\overline{x_{1} x_{2} \ldots x_{n-1}}}_{x_{n}}{\overline{x_{1} x_{2} \ldots x_{n}}}^{-1} .
$$

Note que introduzimos entre os termos $x_{i}$ e $x_{i+1}$ o elemento ${\overline{x_{1} x_{2} \ldots x_{i}}}^{-1} \overline{x_{1} x_{2} \ldots x_{i}}$, onde $1 \leq n-1$. O último elemento na reescrita de $h$ é introduzido visto que $\overline{x_{1} \ldots x_{n}}=$ $\bar{h}=1$. Como cada elemento neste produto é um elemento do conjunto $A$, temos que $A$ gera $H$.

Agora, faremos uma decomposição do conjunto $A$. Seja $B=\left\{u x \overline{u x}^{-1} \mid u \in U, x \in\right.$ $X, u x \notin U\}$. Temos o seguinte lema:

Lema 1.21. Os conjuntos $B^{\prime}=\left\{u x \overline{u x}^{-1} \mid u \in U, x \in X^{-1}, u x \notin U\right\}$ e $B^{-1}=\left\{b^{-1} \mid b \in\right.$ $B\}$ coincidem e $A \backslash\{1\}=B \dot{\cup} B^{-1}$.

Demonstração. Para todo $u \in U$ e $x \in X \cup X^{-1}$, temos $(u x \overline{u x})^{-1}=\overline{u x} x^{-1} u^{-1}=$ $\overline{u x} x^{-1} \overline{\overline{u x} x^{-1}}$, pois $u=\overline{\overline{u x} x^{-1}}$.

Agora, note que $u x \notin U \Leftrightarrow u x \neq \overline{u x} \Leftrightarrow u \neq \overline{u x} x^{-1} \Leftrightarrow \overline{\overline{u x} x^{-1}} \neq \overline{u x} x^{-1} \Leftrightarrow \overline{u x} x^{-1} \notin U$. Tomando $x \in X$, segue que $B^{-1} \subseteq B^{\prime}$ e $B^{\prime-1} \subseteq B$, então $B^{\prime} \subseteq B^{-1}$ e, assim, $B^{\prime}=B^{-1}$. Portanto, da definição de $A$, segue que $A \backslash\{1\}=B \cup \dot{\cup} B^{-1}$.

Agora verificaremos que o conjunto $B$ é uma base para o subgrupo $H$ do grupo livre $F$. Para isso, sejam $b=u x \overline{u x}^{-1}$ e $b^{\prime}=v y \overline{v y}^{-1} \in B \cup B^{-1}=A \backslash\{1\}$. Note que no processo de redução do produto desses elementos para uma palavra reduzida em $X \cup X^{-1}$, obtemos que os fatores do meio não podem ser cancelados, exceto quando $b^{\prime}=b^{-1}$. No lema abaixo veremos o que será o produto desses elementos.

Lema 1.22. Dados $b$ e $b^{\prime}$, o produto $x \overline{u x}^{-1} v y$ é igual a uma palavra reduzida xwy em $X \cup X^{-1}$ para algum $\omega \in F$, exceto quando $v=\overline{u x}, y=x^{-1}$ e $u=\overline{v y}$. 
Demonstração. Sejam $\overline{u x}=x_{1} x_{2} \ldots x_{n}$ e $v=y_{1} y_{2} \ldots y_{m}$ palavras reduzidas em $X \cup X^{-1}$. Observe que no produto $x \overline{u x} \bar{x}^{-1} v y=x x_{n}^{-1} \ldots x_{1}^{-1} y_{1} \ldots y_{m} y$ devemos ter $y_{m} \neq y^{-1}$, pois do contrário $v y=y_{1} \ldots y_{m} y=y_{1} \ldots y_{m-1} \in U$, pois $v \in U$, que é um transversal de Schreier. Logo, $b^{\prime}=v y \overline{v y}^{-1}=v y(v y)^{-1}=1$, o que não pode ocorrer.

Do mesmo modo, devemos ter $x_{n} \neq x$, pois do contrário $u x=\overline{\overline{u x} x^{-1}} x=\overline{x_{1} x_{2} \ldots x_{n-1}} x_{n}=$ $x_{1} x_{2} \ldots x_{n}=\overline{u x} \in U$. Daí teríamos $b=u x \overline{u x}^{-1}=1$, o que não é possível.

Desta maneira, a forma reduzida do produto $x \overline{u x}^{-1} v y$ tem final $y$, pois do contrário $v y=y_{1} y_{2} \ldots y_{m} y$ seria um segmento inicial de $\overline{u x}$ e, assim, $v y \in U$ pela propriedade de Schreier. Assim como, tem início $x$, senão $\left(x \overline{u x}^{-1}\right)^{-1}=\overline{u x} x^{-1}$ seria um segmento inicial de $v$ e pela propriedade de Schreier teríamos $\overline{u x} x^{-1} \in U$ e, assim, $u x=\overline{\overline{u x} x^{-1}} x=$ $\overline{u x} x^{-1} x=\overline{u x}$, o que implicaria $b=1$.

Portanto, nem $x$ e nem $y$ podem ser cancelados, a menos que um cancele o outro, e neste caso teríamos $v=\overline{u x}, y=x^{-1}$ e então $\overline{v y}=\overline{\overline{u x} x^{-1}}=u$. Logo, o produto $x \overline{u x}^{-1} v y$ é igual a uma palavra reduzida $x \omega y$ em $X \cup X^{-1}$, exceto nos casos citados anteriormente.

Com os resultados acima, já podemos demonstrar o resultado principal dessa seção.

Teorema 1.23 (Nielsen-Schreier). Seja F um grupo livre e $H$ um subgrupo de $F$, então $H$ é livre. Além disso, se $|F: H|=g$ e $\operatorname{rank}(F)=r$ são ambos finitos, $\operatorname{rank}(H)=$ $(r-1) g+1$.

Demonstração. Sejam $X$ um conjunto de geradores livres para o grupo livre $F$ e $U$ um transversal de Schreier para $H$ em $F$. Sabemos que o conjunto $A=\left\{u x \overline{u x}^{-1} \mid x \in\right.$ $\left.X \cup X^{-1}\right\}$ gera $H$ e, pelo Lema 1.21 o conjunto $B=\left\{u x \overline{u x}^{-1} \mid u \in U, x \in X, u x \notin U\right\}$ também gera.

Considere $\omega=b_{1} b_{2} \ldots b_{n}$, onde $b_{i}=u_{i} x_{i}{\overline{u_{i} x_{i}}}^{-1}, 1 \leq i \leq n, n \in \mathbb{N}$, uma palavra reduzida em $B \cup \dot{\cup} B^{-1}$, então $b_{i} b_{i+1}=u_{i} x_{i}{\overline{u_{i} x_{i}}}^{-1} u_{i+1} x_{i+1}{\overline{u_{i+1} x_{i+1}}}^{-1}$, onde $1 \leq i \leq n-1$.

Note que, como $\omega=b_{1} b_{2} \ldots b_{n}$ é uma palavra reduzida, $b_{i} b_{i+1} \neq 1$ e pelo lema anterior, segue que $x_{i}{\overline{u_{i} x_{i}}}^{-1} u_{i+1} x_{i+1}$ é igual a uma palavra reduzida nos elementos de $X \cup X^{-1}$ da forma $x_{i} \ldots x_{i+1}$ de comprimento no mínimo 2. Assim, $b_{1} b_{2} \ldots b_{n}=\ldots x_{1} \ldots x_{2} \ldots x_{n} \ldots$ que é uma palavra reduzida em $X \cup X^{-1}$ de comprimento $n \geq 1$. Então $\omega=b_{1} b_{2} \ldots b_{n} \neq 1$. Logo, pela Proposição 1.13 da caracterização de grupos livres, $H$ é livre sobre $B$. 
Observe que os elementos de $B=\left\{u x \overline{u x}^{-1} \mid u \in U, x \in X, u x \notin U\right\}$ são indexados por pares $(u, x) \in U \times X$, com $u x \notin U$ e pelo Lema 1.22 são todos distintos. Então, contando os elementos da base que geram $H$ devemos ter $r(H)=g r-b$, onde $b$ é a quantidade de elementos do conjunto $\{(u, x, v) \in U \times X \times U \mid u x=v\}$ que precisam ser descontados. Vamos mostrar que $b=g-1$.

Consideremos um grafo $T$ com $g$ vértices indexados com os elementos de $U$ e tendo uma aresta $x$ direcionada de $u$ para $v$, tal que $u x=v$. Note que, como $U$ tem a propriedade de Schreier, $T$ é um grafo conexo, ou seja, todo vértice é conectado por um caminho para 1. Além disso, $F$ é livre sobre $X$, então cada palavra de comprimento positivo é diferente de 1 e assim, o grafo $T$ não possui caminhos fechados (as definições sobre grafos podem ser encontradas no próximo capítulo). Pela Definição 2.4 do próximo capítulo, $T$ é uma árvore com $g$ vértices. Pela fórmula de Euler para grafos planares, como $T$ é um grafo conexo, temos $V-A+F=1$, onde $V$ é o número de vértices, $A$ é o números de arestas e $F$ é o número de faces. Como a representação plana de uma árvore não possui faces e $T$ possui $g$ vértices, segue que $A=g-1$.

Note que as arestas de $T$ estão em correspondência biunívoca com os membros do conjunto $\{(u, x, v) \in U \times X \times U \mid u x=v\}$, então segue que $b=g-1$ como queríamos e, portanto, $\operatorname{rank}(H)=g r-(g-1)=(r-1) g+1$.

Agora, quando o subgrupo do grupo livre possui índice infinito, não há uma fórmula para seu posto, mas temos o seguinte resultado:

Corolário 1.24. Seja $H$ um subgrupo de um grupo livre $F$ com base $X$, tal que $\mid F$ : $H \mid=\infty$ e suponha ainda que $H$ contém um subgrupo normal não trivial de F. Então $\operatorname{rank}(H)=\infty$.

Demonstração. Seja $U$ um transversal de Schreier para $H$ em $F$ e $1 \neq \omega=x_{1} x_{2} \ldots x_{n} \in$ $N \subseteq H$. Então, para cada $u \in U$ temos $H u \omega=H u \omega u^{-1} u=H u$, pois $\omega \in N$ e $N \triangleleft F$.

Assim, $\overline{u \omega}=u \neq u \omega$ e $u \omega \notin U$. Portanto, existe um menor $k, 1 \leq k \leq n$ para o qual $u x_{1} x_{2} \ldots x_{k} \notin U$ e $u_{k}=u x_{1} x_{2} \ldots x_{k-1} \in U$. Agora, associamos para cada $u$ um gerador de Schreier $u_{k} x_{k}{\overline{u_{k} x_{k}}}^{-1}$ de $H$ e então $k$ é uma função de $u \in U$, tomando valores em um conjunto finito $\{1,2, \ldots, n\}$. 
Como $|U|=|F: H|$ é infinito, pelo menos uma de suas pré-imagens é infinita, ou seja, existe um conjunto infinito $V \subseteq U$ tal que para todo $v \in V$, o gerador de Scheier de $H$ construído é $v_{k} x_{k}{\overline{v_{k}} x_{k}}^{-1}$ para o mesmo valor de $k$. Se dois destes são iguais, por exemplo, $v_{k} x_{k}{\overline{v_{k} x_{k}}}^{-1}=v_{k}^{\prime} x_{k}{\overline{v_{k}^{\prime} x_{k}}}^{-1}$, para $v, v^{\prime} \in V$, pelo Lema 1.22 temos $v_{k}=v_{k}^{\prime}$ e então $v=v^{\prime}$. Assim, nessa construção temos uma quantidade infinita de geradores distintos para $H$. Logo, $H$ tem posto infinito.

Observação 1.25. A demonstração de que todo subgrupo de um grupo livre é livre pode ser feita também usando recobrimento de grafos, o que veremos mais adiante.

Agora, já podemos afirmar que dados dois subgrupos $A$ e $B$ do grupo livre $F$, ambos serão grupos livres e a interseção $N=A \cap B$ também será um grupo livre. Então, podemos nos questionar sobre o posto desta interseção. Vejamos um exemplo simples:

Exemplo 1.26. Sejam $A=\left\langle a^{2}, b\right\rangle$ e $B=\left\langle a^{2}, b^{a^{-1}}\right\rangle$ subgrupos do grupo livre $F=F(a, b)$. Então, a interseção dos subgrupos $A$ e $B$ será o grupo livre $N=A \cap B=\left\langle a^{2}\right\rangle$ de posto 1 .

No caso deste exemplo é fácil determinar o posto da interseção dos subgrupos livres $A$ e $B$. Mas, no geral, determinar a quantidade de geradores necessários para o posto da interseção não é uma tarefa simples. Neste sentido, Hanna Neumann provou, em 1957, que dados dois subgrupos finitamente gerados $A$ e $B$ de um grupo livre temos:

$$
0 \leqslant \operatorname{rank}(A \cap B)-1 \leqslant 2(\operatorname{rank}(A)-1)(\operatorname{rank}(B)-1) .
$$

Além disso, ela conjecturou que o número 2 na desigualdade acima podia ser removido.

O teorema provado por Hanna Neumann, a conjectura e sua demonstração serão apresentados mais adiante, pois primeiro serão necessários conhecimentos sobre teoria de grafos. 


\section{Capítulo 2}

\section{Grafos}

Definição 2.1. Um grafo $X$ consiste de um conjunto não vazio de vértices $V^{X}$, de um conjunto de arestas $E^{X}$ e de três aplicações $\alpha: E^{X} \rightarrow V^{X}, \omega: E^{X} \rightarrow V^{X},-: E^{X} \rightarrow E^{X}$ tais que, se $e \in E^{X}$, então $\overline{\bar{e}}=e, \bar{e} \neq e$ e $\alpha(e)=\omega(\bar{e})$.

Na definição acima as aplicações representam, respectivamente, o começo, o final e o inverso de uma aresta. Assim, $\alpha(e)$ é chamado o vértice inicial e $\omega(e)$ é chamado o vértice final da aresta $e$. Além disso, podemos ver $X$ como a união $V^{X} \cup E^{X}$ e o grafo será finito se o conjunto de vértices e arestas é finito.

Exemplo 2.2. Exemplos de dois tipos diferentes de grafos:

- $C_{n}, n \in \mathbb{Z}, n \geq 1$ : Seus vértices são os números $0,1, \ldots, n-1$ e suas arestas são os simbolos $e_{i}$ e $\overline{e_{i}}, 0 \leq i \leq n-1$, onde $\alpha\left(e_{i}\right)=i$ e $\omega\left(e_{i}\right)=i+1$;

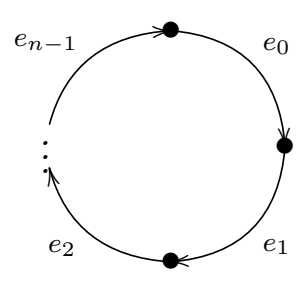

- $C_{\infty}$ : Seus vértices são inteiros e as arestas são os símbolos $e_{i} e \overline{e_{i}}, i \in \mathbb{Z}$, onde $\alpha\left(e_{i}\right)=i$ e $\omega\left(e_{i}\right)=i+1$;

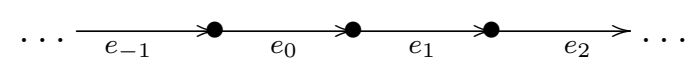


Definição 2.3. O produto direto de grafos $X$ e $Y$ é o grafo $X \times Y$ com conjunto de vértices $V^{X} \times V^{Y}$ e conjunto de arestas $E^{X} \times E^{Y}$ tal que, para $\left(e, e^{\prime}\right) \in E^{X} \times E^{Y}$, temos $\alpha\left(\left(e, e^{\prime}\right)\right)=\left(\alpha(e), \alpha\left(e^{\prime}\right)\right), \omega\left(\left(e, e^{\prime}\right)\right)=\left(\omega(e), \omega\left(e^{\prime}\right)\right) e \overline{\left(e, e^{\prime}\right)}=\left(\bar{e}, \overline{e^{\prime}}\right)$.

Agora, dado um subconjunto $S \subseteq X$, sejam $V^{S}=S \cap V^{X}$ e $E^{S}=S \cap E^{X}$, então se para $e \in E^{S}$ temos $\alpha(e), \omega(e) \in V^{S}$, dizemos que $S$ é subgrafo de $X$. Além disso, para um subconjunto $E \subseteq E^{X}$, podemos definir também o grafo relativo de $X$ como sendo $X(E):=V^{X} \cup E$.

Podemos dar algumas definições gerais para os grafos:

Definição 2.4. Para o grafo $X$, temos:

i) Se ocorrer $\alpha(e)=\omega(e)$, então a aresta e é chamada de laço;

ii) Um caminho em $X$ é uma sequência $p=e_{1} e_{2} \ldots e_{n}$ de arestas, onde $\omega\left(e_{i}\right)=\alpha\left(e_{i+1}\right)$ para $i=1, \ldots, n-1$. Um caminho é dito simples se todos os $\alpha\left(e_{i}\right)$ são distintos;

iii) Um vértice $v \in X$ é um caminho degenerado de comprimento zero;

iv) Um caminho é reduzido se é degenerado ou se $\overline{e_{i}} \neq e_{i+1}$, para $i=1, \ldots, n-1$;

v) Se o final de um caminho $p=e_{1} e_{2} \ldots e_{k}$ coincide com o começo de um caminho $p^{\prime}=e_{1}^{\prime} e_{2}^{\prime} \ldots e_{n}^{\prime}$, então o produto desses caminhos é dado por $p p^{\prime}=e_{1} e_{2} \ldots e_{k} e_{1}^{\prime} \ldots e_{n}^{\prime}$;

vi) Um circuito para um vértice $v \in X$ é um caminho reduzido de v para ele mesmo de comprimento pelo menos um. Esse caminho também pode ser dito um caminho fechado.

Dada a definição de caminho no grafo $X$, podemos definir quando um grafo é conexo e, em seguida, dar a definição de árvore.

Definição 2.5. O grafo $X$ é conexo se existe um caminho conectando quaisquer dois de seus vértices. Além disso, uma componente conexa de X será um subgrafo maximal conexo de $X$ e será denotada por $\operatorname{Comp}(X)$.

Definição 2.6. Um grafo que não possui circuitos é dito uma floresta. E uma floresta conexa é dita uma árvore. 
Note que em uma árvore $T$ do grafo $X$, para quaisquer dois vértices $x, y \in T$, existe um único caminho reduzido de $x$ para $y$. De fato, se $p_{1}$ e $p_{2}$ são dois caminhos distintos de $x$ para $y$, então $p_{1} \overline{p_{2}}$ é um laço, o que não pode ocorrer. Além disso, pode-se garantir a existência de uma subárvore maximal em um grafo conexo.

Lema 2.7. Todo grafo conexo $X$ possui uma subárvore maximal $T$.

Demonstração. Suponha primeiro que $X$ é um grafo finito. Considere uma aresta $e$ em $X$. Adicione arestas a essa aresta inicial de maneira a não formar circuitos. Quando não for mais possível adicionar arestas, pare. O grafo resultante é a subárvore maximal.

Para o caso em que $X$ é infinito, basta considerar uma cadeia de subárvores inclusas e pelo Lema de Zorn existirá um elemento maximal.

Da maneira como a subárvore maximal foi construída, ela conterá todos os vértices do grafo em que está contida:

Proposição 2.8. Seja T uma subárvore maximal de um grafo conexo X. Então T contém todos os vértices de $X$.

Demonstração. Suponha, por contradição, que $T$ não contém todos os vértices de $X$. Então, pela conexidade de $X$, existe uma aresta $y$ começando em $T$ e terminando fora de $T$. Mas, se adicionarmos a $T$ as arestas $y$ e $\bar{y}$ e o vértice $\omega(y)$, obtemos uma árvore $T^{\prime}$ maior do que $T$, contradizendo o fato de $T$ ser maximal. Portanto, $T$ contém todos os vértices de $X$.

Podemos definir a orientação de um grafo a partir da escolha de uma direção de suas arestas.

Definição 2.9. Um grafo $X$ é dito orientado se em cada par de arestas mutuamente inversas $\{e, \bar{e}\}$ uma aresta é escolhida. Essa aresta é chamada positivamente orientada e a outra é dita negativamente orientada. O conjunto das arestas orientadas positivamente é denotado por $E_{+}^{X}$. Esse conjunto é chamado orientação do grafo $X$.

Exemplo 2.10. O buquê de $n$ laços é um grafo com um vértice e $n$ arestas orientadas $e_{1}, e_{2}, \ldots, e_{n}$. 


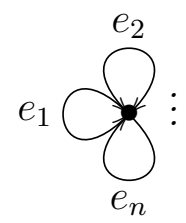

Definida uma orientação para um grafo conexo $X$, podemos mostrar que a quantidade de arestas que não pertencem à subárvore maximal contida em $X$ não depende da escolha dessa árvore. Além disso, é possível calcular essa quantidade.

Lema 2.11. A cardinalidade do conjunto de arestas de um grafo conexo $X$ que estão fora de uma subárvore maximal $T$ não depende da escolha de $T$. Além disso, se $X$ é um grafo conexo finito com orientação $E_{+}^{X}$, então o número de arestas positivamente orientadas de $X$ não pertencendo a $T$ é igual a $\left|E^{X}\right|-\left|V^{X}\right|+1$.

Demonstração. Seja $X$ um grafo conexo com $c_{k}$ caminhos fechados, onde $k=1,2, \ldots$ Para cada $c_{k}$, considere que $c_{k}$ possua $i$ arestas, então o número de arestas que estão na árvore maximal é $i-1$. Note que tenho $i$ maneiras diferentes de considerar essas arestas, o que independe da escolha da árvore maximal $T$.

Agora, vamos calcular o valor de $\left|E^{X}\right|-\left|E^{T}\right|$. Pela fórmula de Euler para grafos planares, como $X$ é um grafo conexo e a representação plana de uma árvore não possui faces, obtemos que $E^{T}=V^{T}-1$. Além disso, pela Proposição 2.8, o número de vértices de $T$ e de $X$ são o mesmo, então obtemos:

$$
\left|E^{X}\right|-\left|E^{T}\right|=\left|E^{X}\right|-\left(\left|V^{T}\right|-1\right)=\left|E^{X}\right|-\left|V^{X}\right|+1
$$

Observação 2.12. Nas próximas seções veremos que o número de arestas positivamente orientadas de $X$ não pertencendo à subárvore maximal nos fornece exatamente o posto de um grupo livre $F$, que será o grupo fundamental do grafo $X$.

Dada uma orientação $E_{+}^{X}$ para o grafo $X$, podemos definir também uma marcação para as arestas do grafo, da seguinte forma:

Definição 2.13. Seja $S$ um alfabeto. Uma marca é uma função $\lambda: E_{+}^{X} \rightarrow S \cup S^{-1}$ tal que para toda aresta $e \in E_{+}^{X}$, temos $\lambda(e)=s$ e $\lambda(\bar{e})=s^{-1}$, para $s \in S$. 
Agora, dado um subgrupo finitamente gerado $H$ de um grupo livre $F$ de base $S$ e um conjunto de geradores para $H$, vamos construir o grafo de $H$.

Construção do grafo do subgrupo. Primeiro considera-se um vértice base $v_{0}$. Para cada gerador do subgrupo finitamente gerado $H$, considere um caminho que sai e retorna para $v_{0}$ e que será composto pelas arestas marcadas que formam o gerador de $H$. As arestas serão marcadas pelos geradores do grupo livre $F$ e nessa construção deve-se observar que duas arestas saindo de um mesmo vértice não podem ser marcadas pelo mesmo $s \in S$.

Vejamos um exemplo:

Exemplo 2.14. Seja $F=F(a, b)$ grupo livre e $H=\left\langle a, b^{2}, b a b^{-1}\right\rangle$. Então podemos construir o grafo de $H$ :

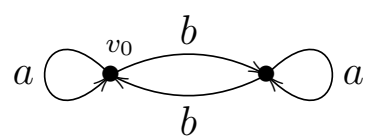

Note que para cada gerador do subrupo $H$, construímos um caminho fechado no grafo a partir do vértice base $v_{0}$.

Agora, se $H$ e $K$ são subgrupos finitamente gerados do grupo livre $F$, podemos construir o grafo da interseção $H \cap K$ a partir dos grafos de $H$ e $K$. Esse grafo será marcado pelos elementos da base de $F$ e seus vértices pertencerão ao conjunto $V^{H} \times V^{K}$. Uma aresta nesse grafo terá, por exemplo, marca $a$ do vértice $(u, v)$ para $\left(u^{\prime}, v^{\prime}\right)$ se, e somente se, o grafo $H$ tem uma $a$-aresta de $u$ para $u^{\prime}$ e o grafo $K$ tem uma $a$-aresta de $v$ para $v^{\prime}$.

Para entender melhor essa construção, vamos ver no exemplo abaixo como construímos o grafo da interseção de dois subgrupos do grupo livre $F=F(a, b)$.

Exemplo 2.15. Sejam $H=\left\langle b a b^{-1}, b^{2}\right\rangle$ e $K=\left\langle a^{2}, b a^{2} b^{-1}\right\rangle$ subgrupos do grupo livre $F=F(a, b)$. Seguindo a construção do grafo do subgrupo descrita acima, primeiro vamos construir os grafos de $H$ e $K$, sendo que o grafo de $H$ terá vértice base $u_{0}$ e o grafo de $K$ terá vértice base $v_{0}$.

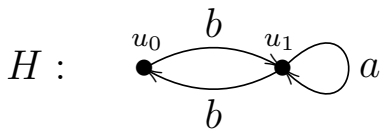

K :

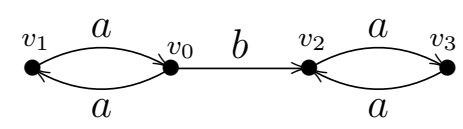


Para a construção do grafo interseção, vamos formar os possíveis pares ordenados com os vértices dos grafos acima:

$$
\left(u_{0}, v_{1}\right),\left(u_{0}, v_{0}\right),\left(u_{0}, v_{2}\right),\left(u_{0}, v_{3}\right),\left(u_{1}, v_{1}\right),\left(u_{1}, v_{0}\right),\left(u_{1}, v_{2}\right),\left(u_{1}, v_{3}\right) .
$$

Esses serão os vértices do grafo interseção $H \cap K$. Em seguida, para descobrir quando eles serão conectados por uma aresta marcada por a ou por b, devemos analisar coordenada a coordenada dos pares.

Por exemplo, considerando os pares $\left(u_{0}, v_{1}\right)$ e $\left(u_{0}, v_{0}\right)$, observe que no grafo $H$ não há nenhuma aresta saindo de $u_{0}$ e retornando para $u_{0}$, enquanto no grafo $K$ tem uma aresta marcada por a saindo de $v_{1}$ até $v_{0}$, logo esses pares não são ligados por nenhuma aresta no grafo da interseção. Agora, considerando os pares $\left(u_{1}, v_{1}\right)$ e $\left(u_{1}, v_{0}\right)$, note que no grafo $H$ temos uma aresta marcada por a saindo e retornando para $u_{1}$, assim como no grafo $K$ temos uma aresta marcada por a saindo de $v_{1}$ e chegando em $v_{0}$, logo esses pares são conectados no grafo da interseção por uma aresta marcada por a saindo de $\left(u_{1}, v_{1}\right)$ e chegando em $\left(u_{1}, v_{0}\right)$. Usando o mesmo argumento teremos uma aresta marcada por a saindo de $\left(u_{1}, v_{0}\right)$ e chegando em $\left(u_{1}, v_{1}\right)$. Após fazer essa análise com todos os pares de vértices, o grafo obtido será:

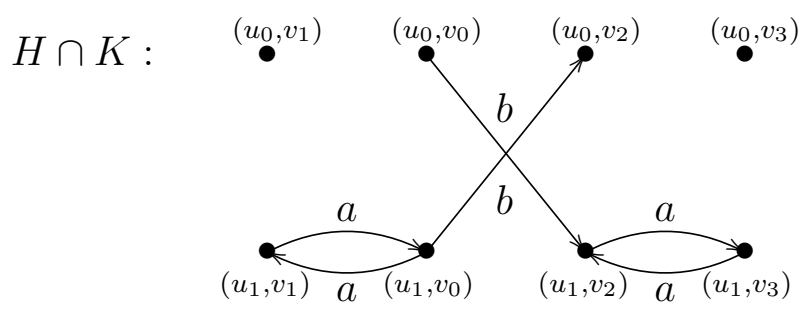

\subsection{Morfismo de grafos}

Definição 2.16. Dados $X$ e $Y$ grafos, um morfismo $f: X \rightarrow Y$ é uma aplicação do conjunto de vértices e arestas de $X$ no conjunto de vértices e arestas de $Y$, levando vértices em vértices e arestas em arestas, e satisfazendo $f(\alpha(e))=\alpha(f(e)), f(\omega(e))=\omega(f(e))$, $f(\bar{e})=\overline{f(e)}$.

A partir da definição acima, um morfismo bijetivo é chamado de isormorfismo e um isomorfismo de um grafo nele mesmo é chamado de automorfismo. Além disso, podemos 
distinguir um vértice do grafo $X$ e escrever $(X, x)$, assim escrevemos $f:(X, x) \rightarrow(Y, y)$, se $f: X \rightarrow Y$ é um morfismo de grafos com $f(x)=y$.

Uma definição importante, e que será bastante utilizada, é a da estrela de um vértice.

Definição 2.17. A estrela de um vértice $x$ em um grafo $X$ é o conjunto de todas as arestas de $X$ com vértice inicial $x$. A valência desse vértice $x$ é a cardinalidade da estrela.

Definição 2.18. Dizemos que um morfismo $f: X \rightarrow Y$ é localmente injetivo se a restrição de $f$ à estrela de qualquer vértice de $X$ é injetiva. De maneira análoga, dizemos que um morfismo $f: X \rightarrow Y$ é localmente sobrejetivo se a restrição de $f$ à estrela de qualquer vértice de $X$ é sobrejetiva.

Se $f: X \rightarrow Y$ é localmente injetivo e sobrejetivo, então $f$ é dito um isomorfismo local.

Exemplo 2.19. Os morfismos abaixo são isomorfismos locais:
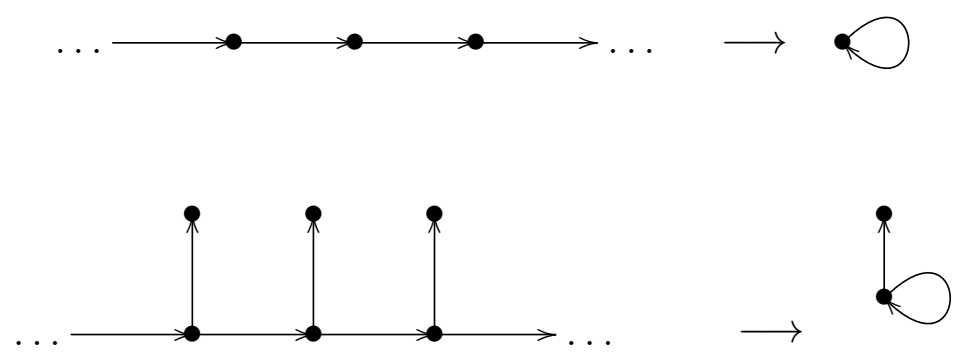

Note que, em ambos os casos, os morfismos restritos à estrela de cada vértice dos grafos do lado esquerdo são localmente injetivos e sobrejetivos.

Observação 2.20. Um morfismo $f: X \rightarrow Y$ localmente injetivo é chamado também de imersão. Além disso, um isomorfismo local entre dois grafos conexos é dito um recobrimento, o qual será descrito melhor em uma seção posterior.

Vamos definir agora pullbacks na categoria de grafos, que são de fácil construção e que serão úteis no nosso resultado proncipal:

Definição 2.21. Sejam $f_{1}: X_{1} \rightarrow Y$ e $f_{2}: X_{2} \rightarrow Y$ morfismos de grafos. Defina $X_{3}$ com o conjunto de vértices $V^{X_{3}}:=\left\{(u, v) \mid u \in V^{X_{1}}, v \in V^{X_{2}}, f_{1}(u)=f_{2}(v)\right\} e$ $o$ conjunto de arestas $E^{X_{3}}:=\left\{\left(e_{1}, e_{2}\right) \mid e_{1} \in E^{X_{1}}, e_{2} \in E^{X_{2}}, f_{1}\left(e_{1}\right)=f_{2}\left(e_{2}\right)\right\}$. Então, 
existirão aplicações de $X_{3}$ para $X_{1}$ e $X_{2}$ que, junto com $f_{1}$ e $f_{2}$, formarão um diagrama pullback:

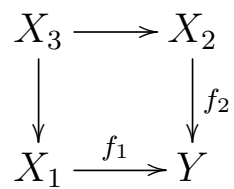

Observação 2.22. Na definição acima, as aplicações de $X_{3}$ para $X_{1}$ e $X_{2}$ são aplicações projeção. Nomeando-as por $\alpha: X_{3} \rightarrow X_{1}$ e $\beta: X_{3} \rightarrow X_{2}$, pela maneira como $X_{3}$ foi considerado, temos que $f_{1} \circ \alpha=f_{2} \circ \beta$.

Note que o pullback é um subgrafo do grafo produto direto $X_{1} \times X_{2}$. A prova da existência de pullbacks pode ser encontrada em [15].

\subsection{Ação de grupos em grafos}

Agora, dado um grupo $\Gamma$ qualquer, definiremos a ação de $\Gamma$ em um grafo $X$.

Definição 2.23. Um grupo $\Gamma$ age em um grafo $X$, se as ações de $\Gamma$ nos conjuntos $V^{X}$ e $E^{X}$ são definidas como $g \cdot \alpha(e)=\alpha(g e), g \cdot \omega(e)=\omega(g e)$ e $g \cdot \bar{e}=\overline{g e}$, para todo $g \in \Gamma e e \in E^{X}$.

Dizemos que $\Gamma$ age em $X$ sem inversão de arestas se $g \cdot e \neq \bar{e}$ para todo $g \in \Gamma e$ $e \in E^{X}$. E a ação é dita livre se $g \cdot v \neq v, \forall v \in V^{X}$ e $1 \neq g \in \Gamma$.

Observação 2.24. Note que a ação definida acima é pela esquerda e, neste caso, diremos que $X$ é um $\Gamma$-grafo.

Podemos sempre considerar que $\Gamma$ age sem inversão de arestas em $X$. De fato, caso contrário, considere sua subdivisão baricêntrica: uma subdivisão baricêntrica de um grafo $X$ é um grafo $B(X)$ que pode ser obtido de $X$ por uma subdivisão de cada aresta $e$ em duas arestas $e_{1}$ e $e_{2}$ e adicionando um novo vértice $v_{e}$, correspondendo ao meio da aresta $e$
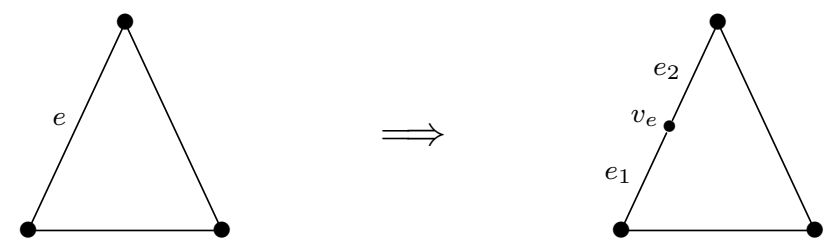
Assumimos que $(\bar{e})_{2}=\overline{e_{1}},(\bar{e})_{1}=\overline{e_{2}}, v_{e}=v_{\bar{e}}$ e definimos a ação de $\Gamma$ no grafo $B(X)$ colocando $g \cdot e_{1}=(g e)_{1}, g \cdot e_{2}=(g e)_{2}, g \cdot v_{e}=v_{g e}$ e preservando a ação nos vértices do grafo $B(X)$. Assim como definido, $\Gamma$ age sem inversão de arestas na sua subdivisão baricêntrica:

$$
\begin{aligned}
& g \cdot e_{1}=(g e)_{1}=\overline{(\overline{g e})_{1}}=g \overline{(\bar{e})_{1}}=g \overline{\left(\overline{e_{2}}\right)}=g e_{2} \neq \overline{e_{1}} \\
& g \cdot e_{2}=(g e)_{2}=\overline{(\overline{g e})_{2}}=g \overline{(\bar{e})_{2}}=g \overline{\left(\overline{e_{1}}\right)}=g e_{1} \neq \overline{e_{2}}
\end{aligned}
$$

Agora, considere $\Gamma$ um grupo agindo em um grafo $X$ sem inversão de arestas. Para $x \in V^{X} \cup E^{X}$, podemos definir o estabilizador de $x$ como sendo o conjunto

$$
\Gamma_{x}=\{g x=x \mid g \in \Gamma\}
$$

e a órbita de $x$ como sendo o conjunto

$$
\Gamma x=\{g x \mid g \in \Gamma\} .
$$

A partir disso, definimos o grafo quociente $\Gamma \backslash X$ :

Definição 2.25. O grafo quociente $\Gamma \backslash X$ é o grafo com conjunto de vértices $\Gamma v$, onde $v \in V^{X}$ e conjunto de arestas $\Gamma$, onde $e \in E^{X}$, tal que $\Gamma v$ é o começo de $\Gamma$ e se existe $g \in \Gamma$ tal que gv é o começo de e e o inverso da aresta $\Gamma e$ é a aresta $\Gamma \bar{e}$.

Definição 2.26. A aplicação $f: X \rightarrow \Gamma \backslash X$ dada por $f(x)=\Gamma x$, onde $x \in V^{X} \cup E^{X}$, é um morfismo de grafos chamado de projeção ou aplicação quociente. E se y é um vértice ou aresta do grafo $\Gamma \backslash X$, qualquer pré-imagem de y com respeito a $f$ é chamada de levantamento de y em $X$.

Proposição 2.27. Seja $\Gamma$ um grupo agindo em um grafo conexo $X$. Para qualquer subárvore $T^{\prime}$ do grafo quociente $\Gamma \backslash X$, existe uma subárvore $T$ em $X$ tal que $\left.f\right|_{T}: T \rightarrow T^{\prime}$ é um isomorfismo.

Demonstração. Temos $f: X \rightarrow \Gamma \backslash X$ um morfismo de grafos. Considere o conjunto de todas as subárvores em $X$ projetadas injetivamente em $T^{\prime}$. Note que esse conjunto é parcialmente ordenado por inclusão e qualquer cadeia de seus elementos tem um limite superior, que será a união das árvores. Pelo Lema de Zorn, esse conjunto tem um elemento maximal $T$. Basta provar então que $f(T)=T^{\prime}$. Suponha que $f(T) \neq T^{\prime}$. Então, existe 
uma aresta $e^{\prime}$ com ponto inicial em $f(T)$ e ponto final no complemento $T^{\prime}-f(T)$. Assim, existirá um levantamento de $e^{\prime}$ em $T$ e então $T$ pode ser aumentado, uma contradição. Logo $f(T)=T^{\prime}$.

Observação 2.28. Qualquer subárvore T como na proposição acima, é dita um levantamento em $X$ da subárvore $T^{\prime}$.

Agora, veremos a definição de um tipo especial de grafo, o grafo de Cayley:

Definição 2.29. O Grafo de Cayley de $\Gamma$ com respeito a um subconjunto $S$ de $\Gamma$, denotado por $X(\Gamma, S)$, é o grafo com conjunto de vértices $\Gamma$, conjunto de arestas positivamente ordenadas $\Gamma \times S$, e funções $\alpha, \omega$ tais que $\alpha((g, s))=g$ e $\omega((g, s))=g s$.

Assim, se $e=(g, s)$ é uma aresta de $X(\Gamma, S)$, teremos $\bar{e}=\left(g s, s^{-1}\right)$ e a marca da aresta será $\lambda(e)=s$. Além disso, o grupo $\Gamma$ age por multiplicação à esquerda em $X(\Gamma, S)$ : dado $g^{\prime}$ vértice e $\left(g^{\prime}, s\right)$ aresta de $X(\Gamma, S)$, temos $g \cdot g^{\prime}=g g^{\prime}$ e $g \cdot\left(g^{\prime}, s\right)=\left(g g^{\prime}, s\right)$.

Note que essa ação é livre, pois $\forall g^{\prime}$ vértice de $X(\Gamma, S)$ e $1 \neq g \in \Gamma$, temos $g \cdot g^{\prime}=$ $g g^{\prime} \neq g^{\prime}$. E é sem inversão de arestas, pois dado $g \in \Gamma$ e $\left(g^{\prime}, s\right) \in \Gamma \times S$, temos $g \cdot\left(g^{\prime}, s\right)=$ $\left(g g^{\prime}, s\right) \neq\left(g^{\prime} s, s^{-1}\right)=\overline{\left(g^{\prime}, s\right)}$.

Exemplo 2.30. Vejamos alguns exemplos:

1. Dado $D_{3}=<\rho, \tau \mid \rho^{3}=1, \tau^{2}=1, \tau \rho^{2}=\rho \tau>e S=\{\rho, \tau\}$, podemos definir o grafo de Cayley $X\left(D_{3}, S\right)$, onde as arestas pertencem a $D_{3} \times S$ e os vértices pertencem a $D_{3}$.

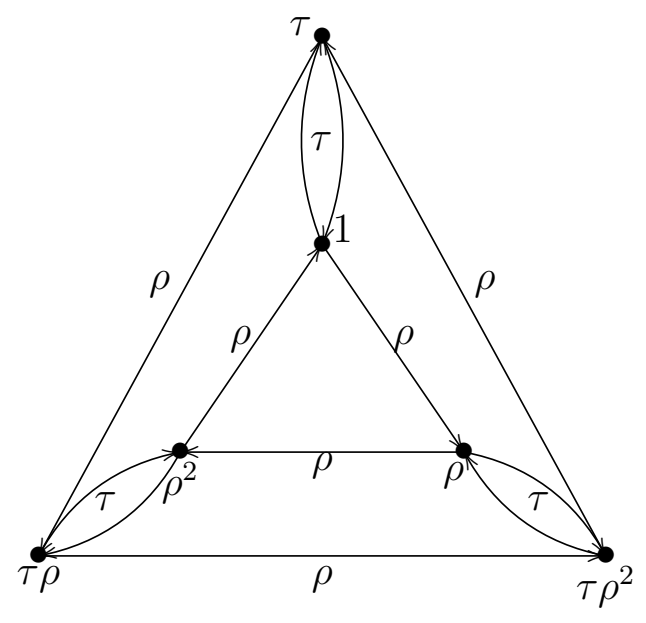


2. De modo análogo, dado $D_{4}=<\rho, \tau \mid \rho^{4}=1, \tau^{2}=1, \tau \rho^{3}=\rho \tau>$ e $S=\{\rho, \tau\}$, definimos o grafo de Cayley $X\left(D_{4}, S\right)$, onde as arestas pertencem a $D_{4} \times S$ e os vértices a $D_{4}$.

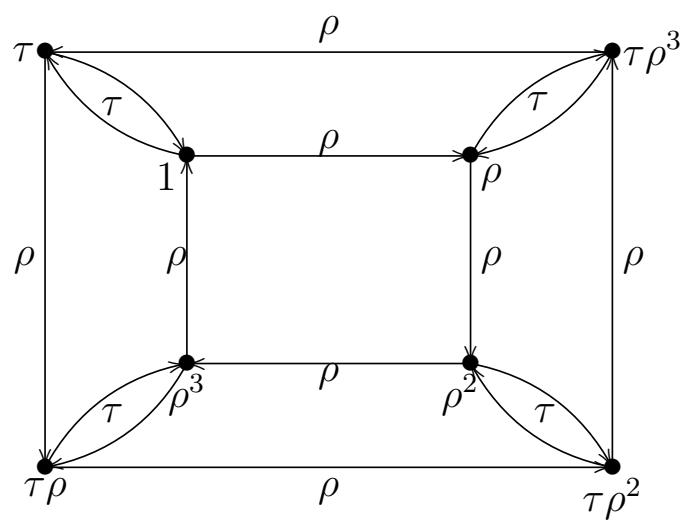

3. Seja $F=F(x, y)$ grupo livre de dois elementos. O grafo de Cayley com respeito ao conjunto de geradores $S=\{x, y\}$, será dado por:

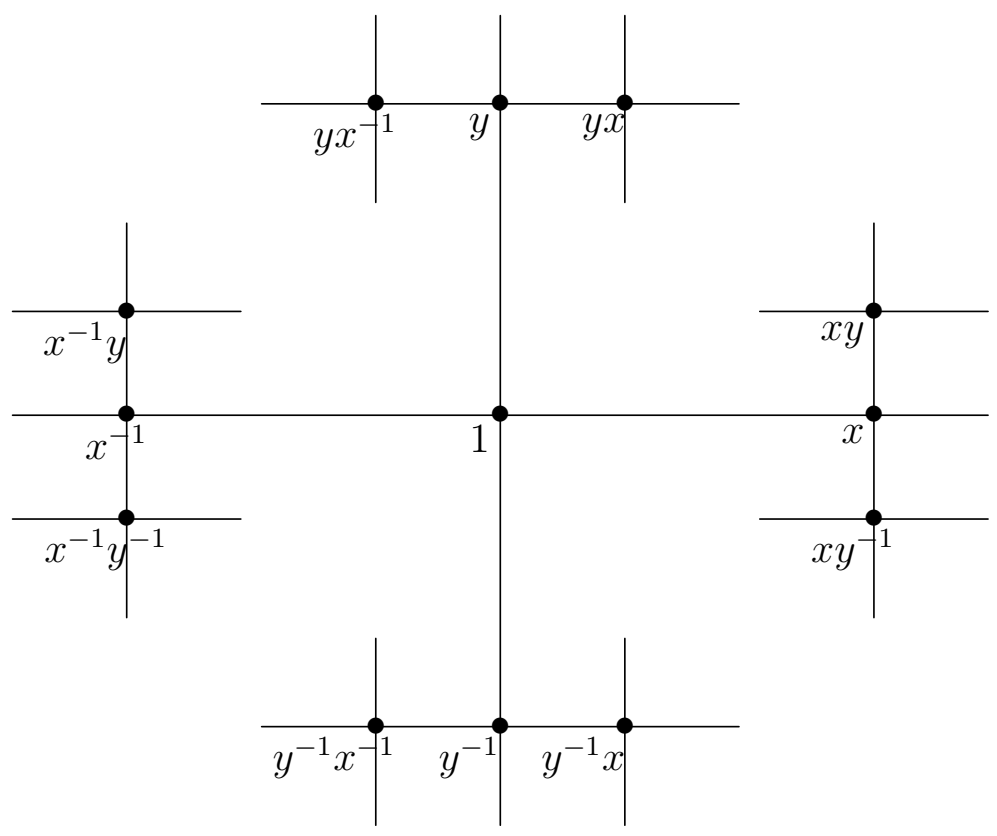

Lema 2.31. O grafo $X(\Gamma, S)$ é conexo se, e somente se, o subconjunto $S$ gera $\Gamma$.

Demonstração. Se $X(\Gamma, S)$ é um grafo conexo, então todos os seus vértices, elementos de $\Gamma$, são ligados por caminhos. Esses caminhos são sequências de arestas que pertencem a $\Gamma \times S$, ou seja, são combinação dos elementos de $S$ e seus inversos e os vértices são produtos desses elementos. Logo $S$ gera $\Gamma$. 
Reciprocamente, considere que todos os elementos do grupo $\Gamma$ podem ser escritos como produto finito dos elementos de $S$ e seus inversos. Seja $X(\Gamma, S)$ o grafo de Cayley, então seus vértices são elementos de $\Gamma$ e serão ligados por caminhos, ou seja, sequências de arestas pertencentes a $\Gamma \times S$. Portanto $X(\Gamma, S)$ é conexo.

Proposição 2.32. Seja $X(\Gamma, S)$ o grafo de Cayley de $\Gamma$ com respeito ao subconjunto $S$. Então $X(\Gamma, S)$ é uma árvore se, e somente se, $\Gamma$ é um grupo livre com base $S$.

Demonstração. Para cada aresta $e=(g, s)$, com $s \in S \cup S^{-1}$, defina sua marca como $\lambda(e)=s$. Então $\omega(e)=\alpha(e) \lambda(e)$ e para qualquer caminho $e_{1} e_{2} \ldots e_{n} \in X(\Gamma, S)$ temos $\omega\left(e_{n}\right)=\alpha\left(e_{1}\right) \lambda\left(e_{1}\right) \ldots \lambda\left(e_{n}\right)$.

Se $\Gamma$ é um grupo livre com base $S$, então $S$ gera $\Gamma$ e portanto $X(\Gamma, S)$ é conexo pelo resultado anterior. Suponha que em $X(\Gamma, S)$ existe um caminho fechado reduzido $e_{1} e_{2} \ldots e_{n}$. Então $\omega\left(e_{n}\right)=\alpha\left(e_{1}\right), \operatorname{assim} \lambda\left(e_{1}\right) \ldots \lambda\left(e_{n}\right)=1$ e já que $S$ é base de $\Gamma$, existe um índice $k$ tal que $\lambda\left(e_{k}\right)=\lambda\left(e_{k+1}\right)^{-1}$. Então, $e_{k}=\bar{e}_{k+1}$, uma contradição com o fato do caminho ser reduzido. Portanto em $X(\Gamma, S)$ não exitem caminhos fechados reduzidos, ou seja, $X(\Gamma, S)$ é uma árvore.

Por outro lado, se $X(\Gamma, S)$ é uma árvore, então cada palavra em $S \cup S^{-1}$ corresponde a um único vértice no grafo e esse vértice é diferente de 1 . Logo essa palavra é diferente de 1. Como $X(\Gamma, S)$ é conexo, pelo lema anterior $S$ gera $\Gamma$. Portanto pela Proposição 1.13, $\Gamma$ é grupo livre de base $S$.

Corolário 2.33. Qualquer grupo livre age livremente e sem inversão de arestas em uma árvore.

Demonstração. Seja $\Gamma$ um grupo livre com base $S$, então o grafo de Cayley $X(\Gamma, S)$ de $\Gamma$ é uma árvore. Note que $\Gamma$ age por multiplicação à esquerda em $X(\Gamma, S)$ e essa ação é livre e sem inversão de arestas como observado anteriormente.

Outro tipo de grafo que podemos definir é o Grafo de Schreier de um subgrupo do grupo livre.

Definição 2.34. Seja $\Gamma$ um grupo livre de base $S$ e $H$ um subgrupo de $\Gamma$. O Grafo de Schreier de $\boldsymbol{H}$, denotado por $X(H)$, é o grafo de classes laterais à direita de $H$, onde 
$V^{X(H)}=H \backslash \Gamma=\{H g \mid g \in \Gamma\}$ e se $e \in E_{+}^{X(H)}$ então $e=(H g, H g s), \forall H g, \forall s \in S e$ $\lambda(e)=s$.

Dentro de um grafo existe um grupo especial, o chamado grupo fundamental de um grafo, que descreveremos em sequência.

\subsection{Grupo fundamental de um grafo}

Antes de definir o grupo fundamental do grafo $X$ vamos considerar caminhos em $X$ e uma relação de equivalência entre eles, chamada homotopia de caminhos. Mas, primeiro, vamos definir homotopia entre duas aplicações.

Definição 2.35. Se $f$ e $f^{\prime}$ são duas aplicações contínuas do espaço $X$ no espaço $Y$, dizemos que $f$ é homotópica a $f^{\prime}$ se existe uma aplicação contínua $h:[0,1] \times X \rightarrow Y$ tal que $h(0, x)=f(x)$ e $h(1, x)=f^{\prime}(x)$. A aplicação h é chamada de homotopia entre $f$ e $f^{\prime}$.

Além disso, dizemos que dois espaços $X$ e $Y$ são homotopicamente equivalentes se existem aplicações contínuas $f: X \rightarrow Y$ e $g: Y \rightarrow X$ tais que a aplicação $g \circ f$ é homotópica à aplicação identidade em $X$ e a aplicação $f \circ g$ é homotópica à aplicação identidade em $Y$. Se um espaço é homotopicamente a um ponto, ele é chamado de contrátil.

Exemplo 2.36. Uma árvore é homotopicamente equivalente à um ponto, ou seja, ela é contrátil. Veja o exemplo abaixo:
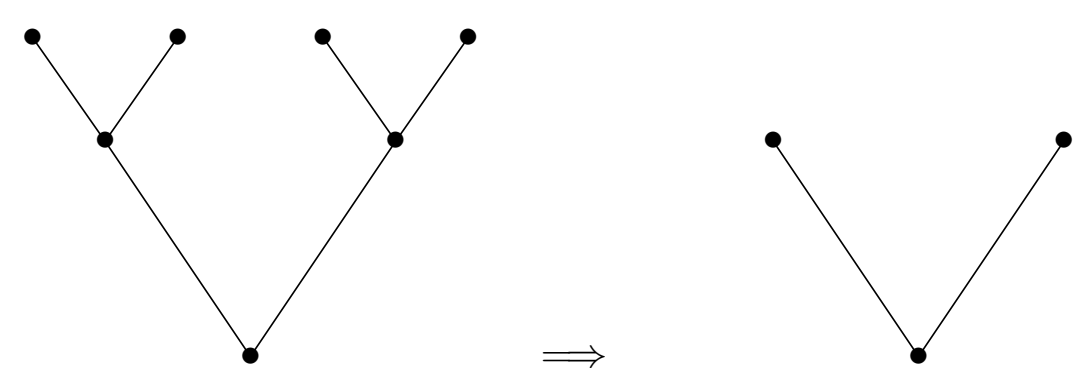

Agora, seja $X$ um grafo com um vértice distinguido $x$. Considere o conjunto $P(X, x)$ de todos os caminhos em $X$ com começo e final em $x$. Para quaisquer dois caminhos 
$p=e_{1} e_{2} \ldots e_{k}$ e $q=e_{1}^{\prime} e_{2}^{\prime} \ldots e_{n}^{\prime}$ de $P(X, x)$, seu produto $p q=e_{1} \ldots e_{k} e_{1}^{\prime} \ldots e_{n}^{\prime}$ também está em $P(X, x)$.

Definição 2.37. Dizemos que os caminhos $p_{1}$ e $p_{2} \in P(X, x)$ são homotópicos se $p_{2}$ pode ser obtido de $p_{1}$ por um número finito de inserções ou deletações de subcaminhos da forma e $\bar{e}$.

A classe de homotopia de um caminho $p \in P(X, x)$, denotada por $[p]$ é o conjunto de todos os caminhos homotópicos a p.

Podemos definir o produto de duas classes de homotopia $[p]$ e $[q]$ como $[p][q]=[p q]$. Esse produto é bem definido, pois adicionando ou deletando subcaminhos da forma $e \bar{e}$ a igualdade é preservada. Além disso, podemos considerar o caminho degenerado $x$ como o elemento identidade e o elemento inverso como sendo $\left[p^{-1}\right]=[p]^{-1}$, pois como no caso do produto, a igualdade será preservada. Assim, está definido o grupo fundamental do grafo $X:$

Definição 2.38. O conjunto das classes de homotopia de caminhos de $P(X, x)$, com respeito ao produto definido acima, forma um grupo chamado de grupo fundamental do grafo $X$ com respeito ao vértice $x$ e é denotado por $\pi_{1}(X, x)$.

Agora, queremos mostrar que o grupo fundamental de um grafo conexo $X$ é livre. Para isso, escolha uma subárvore maximal $T$ em $X$ e um vértice distinguido $x$ em $X$. Para qualquer vértice $v \in V^{X}$ existe um único caminho reduzido em $T$ de $x$ para $v$, denotado por $p_{v}$. Para cada aresta $e \in E^{X}$ associamos o caminho fechado $p_{e}=p_{\alpha(e)} e p_{\omega(e)}^{-1}$.

Teorema 2.39. Sejam $X$ um grafo conexo, $x \in V^{X}$ e $T$ uma subárvore maximal em $X$. Então $\pi_{1}(X, x)$ é um grupo livre com base $S=\left\{\left[p_{e}\right] \mid e \in E_{+}^{X} \backslash E^{T}\right\}$, onde $E_{+}^{X}$ é a orientação de $X$.

Demonstração. Se $p=e_{1} e_{2} \ldots e_{k}$ é um caminho fechado em $X$ começando em $x$, então $[p]=\left[p_{e_{1}}\right]\left[p_{e_{2}}\right] \ldots\left[p_{e_{k}}\right]$, onde cada $p_{e_{i}}=p_{\alpha\left(e_{i}\right)} e_{i} p_{\omega\left(e_{i}\right)}^{-1}$, com $e_{i} \in E^{X}$. Note que se $e_{i} \in E^{T}$, $p_{e_{i}}$ é um caminho na árvore $T$ e então equivalente a $x$, assim $\left[p_{e_{i}}\right]=1$. Portanto, o grupo $\pi_{1}(X, x)$ é gerado pelo conjunto $S$. Resta provar que a forma reduzida dos elementos de $\pi_{1}(X, x)$ com respeito a $S$ é única. 
Seja $[p]=\left[p_{e_{1}}\right]\left[p_{e_{2}}\right] \ldots\left[p_{e_{k}}\right]$ a forma reduzida do elemento $[p]$ com respeito ao conjunto S. Então $e_{i} \in E^{X} \backslash E^{T}$ e $e_{i+1} \neq \bar{e}_{i}$, para todo $i$. Como foi definido, o caminho $p_{e_{i}}$ é levado primeiro na árvore $T$, então ao longo da aresta $e_{i}$, que não pertence à árvore, e finalmente de novo na árvore. Assim, cancelamentos no caminho $p_{e_{1}} p_{e_{2}} \ldots p_{e_{k}}$ não alcançam a aresta $e_{i}$ de $p_{e_{i}}$. Então, o caminho $p$ é homotópico ao caminho reduzido da forma $t_{1} e_{1} t_{2} e_{2} \ldots t_{k} e_{k} t_{k+1}$, onde os caminhos $t_{i}$ estão dentro da árvore $T$. Desde que cada classe de homotopia contém somente um caminho reduzido, a sequência $e_{1} e_{2} \ldots e_{k}$ é unicamente determinada por $[p]$. Então a forma reduzida de $[p]$ é única.

Agora, como definimos no Exemplo 2.10, um buquê de $n$ laços é um grafo conexo, logo seu grupo fundamental é um grupo livre. Esse resultado pode ser enunciado como o teorema abaixo, e a construção de sua demonstração pode ser encontrada no livro clássico de Topologia de John Stillwell [26].

Teorema 2.40. Seja $X$ um buquê com $n$ laços, ou seja, o produto de $n$ cópias de $S^{1}$ indexadas por um elemento. Então $\pi_{1}(X)=F_{n}$.

Assim, podemos concluir que um grupo é livre se, e somente se, é o grupo fundamental de um grafo. De fato, para isso é suficiente notar que todo grafo é homotopicamente equivalente à um buquê: dado $X$ um grafo qualquer, podemos considerar $T$ sua subárvore maximal, que será homotopicamente equivalente a um vértice, e as arestas não pertencentes a $T$ que permanecerão, serão laços anexados a esse um vértice, formando assim um buquê de laços.

Observação 2.41. Com os resultados obtidos acima e pelo Lema 2.11, que faz a contagem do número de arestas de um grafo conexo $X$ que não pertencem a uma subárvore maximal, concluimos que o posto de um grupo livre $F$, que é o grupo fundamental do grafo X, será dado por:

$$
\operatorname{rank}(F)=\operatorname{rank}\left(\pi_{1}(X)\right)=\left|E^{X}\right|-\left|V^{X}\right|+1
$$

\subsection{Recobrimento de grafos}

Definição 2.42. Um morfismo de grafos $f: X \rightarrow Y$ é dito uma aplicação de recobrimento se $f$ leva o conjunto de vértices e arestas de $X$ sobrejetivamente no conjunto de 
vértices e arestas de $Y$, de maneira que a estrela de cada vértice $v \in V^{X}$ é bijetivamente levada na estrela do vértice $f(v)$.

Observação 2.43. Quando X é uma árvore, dizemos que o recobrimento é universal.

Exemplo 2.44. 1) Sejam $C_{n}$ e $C_{\infty}$ os grafos definidos no Exemplo 2.2. Então, para cada $n \geq 1$ existe uma aplicação de recobrimento do grafo $C_{\infty}$ para o grafo $C_{n}$.

2) Seja $X(\Gamma, S)$ o grafo de Cayley do grupo $\Gamma$ com respeito ao conjunto $S$. Qualquer subgrupo $H$ de $\Gamma$ age por multiplicação à esquerda em $X(\Gamma, S)$. Então, a aplicação projeção $X(\Gamma, S) \rightarrow H \backslash X(\Gamma, S)$ é um recobrimento.

Note que o grafo $\{1\} \backslash X(\Gamma, S)$ coincide com $X(\Gamma, S)$, enquanto o grafo $\Gamma \backslash X(\Gamma, S)$ tem um vértice e $|S|$ pares de arestas mutuamente inversas.

Agora, se $f: X \rightarrow Y$ é um recobrimento e $p$ é um caminho em $Y$, então um levantamento do caminho $p$ é qualquer caminho $l$ em $X$ tal que $f(l)=p$. Para qualquer caminho $p$ em $Y$ e para qualquer levantamento $v$ de seu vértice inicial $f(v)$, existe um único levantamento de $p$ começando em $v$. Além disso, se dois caminhos $l_{1}, l_{2} \in X$ são homotópicos, então $f\left(l_{1}\right)$ e $f\left(l_{2}\right)$ são homotópicos. Inversamente, se dois caminhos $p_{1}$ e $p_{2} \in Y$ são homotópicos, então seus levantamentos em $X$ que começam com o mesmo vértice são homotópicos.

Através da definição do produto das classes de homotopia de caminhos, dados $X$ e $Y$ grafos conexos e $f:(X, x) \rightarrow(Y, y)$ um morfismo, podemos definir o homomorfismo $f_{*}: \pi_{1}(X, x) \rightarrow \pi_{1}(Y, y)$, onde $[l] \mapsto[f(l)]$. De fato, dadas classes de homotopia $\left[l_{1}\right],\left[l_{2}\right] \in$ $\pi_{1}(X, x)$, temos $f_{*}\left(\left[l_{1}\right]\left[l_{2}\right]\right)=f_{*}\left(\left[l_{1} l_{2}\right]\right)=f\left(\left[l_{1} l_{2}\right]\right)=f\left(\left[l_{1}\right]\right) f\left(\left[l_{2}\right]\right)=f_{*}\left(\left[l_{1}\right]\right) f_{*}\left(\left[l_{2}\right]\right)$.

Dado $H$ subgrupo de $\pi_{1}(Y, y)$, diremos que o recobrimento $f:(X, x) \rightarrow(Y, y)$ corresponde ao subgrupo $H$ se $f_{*}\left(\pi_{1}(X, x)\right)=H$. A partir disso, o teorema abaixo apresenta resultados interessantes sobre o recobrimento de grafos.

Teorema 2.45. 1. Se $f:(X, x) \rightarrow(Y, y)$ é um recobrimento, então o homomorfismo $f_{*}: \pi_{1}(X, x) \rightarrow \pi_{1}(Y, y)$ é injetivo.

2. Para cada subgrupo $H \leq \pi_{1}(Y, y)$ existe um recobrimento $f:(X, x) \rightarrow(Y, y)$ tal que $f_{*}\left(\pi_{1}(X, x)\right)=H$. 
3. Sejam $f_{1}:\left(X_{1}, x_{1}\right) \rightarrow(Y, y)$ e $f_{2}:\left(X_{2}, x_{2}\right) \rightarrow(Y, y)$ recobrimentos tais que $f_{1 *}\left(\pi_{1}\left(X_{1}, x_{1}\right)\right)=f_{2 *}\left(\pi_{1}\left(X_{2}, x_{2}\right)\right)=H$. Então existe um isomorfismo $p:\left(X_{1}, x_{1}\right) \rightarrow$ $\left(X_{2}, x_{2}\right)$ tal que $f_{1}=f_{2} \circ p$.

4. Seja $f:(X, x) \rightarrow(Y, y)$ um recobrimento. O grafo $X$ é uma árvore se, e somente se, $f_{*}\left(\pi_{1}(X, x)\right)=\{1\}$. Se $X$ é uma árvore, então o grupo $\pi_{1}(Y, y)$ age em $X$ livremente e o grafo quociente $\pi_{1}(Y, y) \backslash X$ é isomorfo ao grafo $Y$.

5. Seja $H$ um subgrupo normal do grupo $\pi_{1}(Y, y)$ e seja $f:(X, x) \rightarrow(Y, y)$ o recobrimento correspondente a $H$. Então o grafo quociente $\pi_{1}(Y, y) \backslash H$ age em $X$ livremente e o grafo quociente por essa ação é isomorfo a $Y$.

Demonstração. $\quad$ 1. Mostraremos que o homomorfismo $f_{*}: \pi_{1}(X, x) \rightarrow \pi_{1}(Y, y)$ é injetivo. Para isso, considere os caminhos degenerados $1_{x}$ e $1_{y}$ em $X$ e $Y$ com vértices iniciais $x$ e $y$, respectivamente. Seja $[l] \in \pi_{1}(X, x)$ e suponha que $f_{*}([l])=[f(l)]=\left[1_{y}\right]$. Desse modo, os caminhos $f(l)$ e $1_{y}$ são homotópicos, o que implica que seus levantamentos $l$ e $1_{x}$ são homotópicos. Portanto, $[l]=1$ e a aplicação é injetiva.

2. Queremos mostrar que existe um recobrimento $f:(X, x) \rightarrow(Y, y)$ que se corresponde ao subgrupo $H$ de $\pi_{1}(Y, y)$. Escolha uma subárvore maximal $T$ em $Y$. Para cada vértice $v \in V^{Y}$ existe um único caminho reduzido indo de $y$ para $v$ na árvore. Denote esse caminho por $p_{v}$ e para cada aresta $e \in E^{Y}$ defina o caminho $p_{e}=p_{\alpha(e)} e p_{\omega(e)}^{-1}$, como definido anteriormente na seção do grupo fundamental.

Seja $\left\{t_{i} \mid i \in I\right\}$ um sistema de representantes das classes laterais de $H$ em $\pi_{1}(Y, y)$. O representante de $H$ será $t_{1}=1$. Considere os conjuntos $V^{X}=\left\{(v, i) \mid v \in V^{Y}, i \in\right.$ $I\}, E^{X}=\left\{(e, i) \mid e \in E^{Y}, i \in I\right.$, tais que $\alpha((e, i))=(\alpha(e), i)$ e $\omega((e, i))=(\omega(e), j)$, onde $j$ é tal que $H t_{j}=H t_{i}\left[p_{e}\right]$ e $\overline{(e, i)}=(\bar{e}, j)$. Distingua o vértice $x=(y, 1)$ no grafo $X$. Então, definimos a aplicação $f: X \rightarrow Y$ tal que $f((v, i))=v, f((e, i))=e$, onde $v \in V^{X}$ e $e \in E^{X}$, que será o recobrimento desejado.

Vamos mostrar que o grafo $X$ é conexo. Para cada $i \in I$, seja $T_{i}$ o subgrafo de $X$ com o conjunto de vértices $V^{T_{i}}=\left\{(v, i) \mid v \in V^{T}\right\}$ e o conjunto de arestas $E^{T_{i}}=\left\{(e, i) \mid e \in E^{T}\right\}$. Note que o grafo $T_{i}$ é isomorfo a $T$ pelo jeito como foi 
definido, então é conexo e $\cup_{i \in I} V^{T_{i}}=V^{X}$. Portanto, é suficiente provar que para quaisquer $i, j \in I$, os grafos $T_{i}$ e $T_{j}$ são conectados por um caminho em $X$.

Seja $g=e_{1} e_{2} \ldots e_{s}$ um caminho em $Y$ com vértice inicial e final $y$, tal que $H t_{i}[g]=$ $H t_{j}$. Então, usando a definição dada antes, a classe de homotopia do caminho $g$ será dada por $[g]=\left[p_{e_{1}}\right]\left[p_{e_{2}}\right] \ldots\left[p_{e_{s}}\right]$. Agora, defina a sequência $\left(i_{1}, i_{2}, \ldots, i_{s+1}\right)$ tal que $H t_{i_{k+1}}=H t_{i_{k}}\left[p e_{k}\right], 1 \leq k \leq s$. Então $i_{s+1}=j$ e o caminho $\left(e_{1}, i_{1}\right)\left(e_{2}, i_{2}\right) \ldots\left(e_{s}, i_{s}\right)$ conecta os vértices $(y, i) \in T_{i}$ e $(y, j) \in T_{j}$. Então, $X$ é conexo e $f: X \rightarrow Y$ é um recobrimento.

Note que um caminho arbitrário $\left(e_{1}, 1\right)\left(e_{2}, i_{2}\right) \ldots\left(e_{s}, i_{s}\right)$ em $X$ com vértice inicial $x=(y, 1)$ é fechado se, e somente se, o caminho $g=e_{1} e_{2} \ldots e_{s}$ em $Y$ é fechado e $H \cdot 1 \cdot\left[p_{e_{1}}\right]\left[p_{e_{2}}\right] \ldots\left[p_{e_{s}}\right]=H \cdot 1$, isto é, $[g] \in H$. Portanto, $f_{*}\left(\pi_{1}(X, x)\right)=H$.

3. Vamos construir um isomorfismo $p$ entre os grafos $X_{1}$ e $X_{2}$. Seja $x$ um vértice arbitrário do grafo $X_{1}$ e escolha um caminho arbitrário $l_{1}$ em $X_{1}$ com vértice inicial $x_{1}$ e vértice terminal $x$. Então, existe um único caminho $l_{2}$ em $X_{2}$ com vértice inicial $x_{2}$ tal que $f_{1}\left(l_{1}\right)=f_{2}\left(l_{2}\right)$. Seja $p(x)$ o vértice terminal de $l_{2}$.

Essa definição não depende da escolha do caminho $l_{1}$. De fato, seja $l_{1}^{\prime}$ um outro caminho em $X_{1}$ com vértice inicial $x_{1}$ e vértice terminal $x$ e $l_{2}^{\prime}$ um caminho em $X_{2}$ com vértce inicial $x_{2}$, tais que $f_{1}\left(l_{1}^{\prime}\right)=f_{2}\left(l_{2}^{\prime}\right)$. Se $l_{1}$ e $l_{1}^{\prime}$ diferem por um caminho fechado $g$, então $g l_{1}$ é homotópico a $l_{1}^{\prime}$ e $f_{1}\left(l_{1}\right)$ e $f_{1}\left(l_{1}^{\prime}\right) \in Y$ diferem também por um caminho cuja classe de homotopia está em $H$. Então, os levantamentos $l_{2}$ e $l_{2}^{\prime}$ desses caminhos em $X_{2}$ também diferem por um caminho fechado e seus vértices terminais coincidem.

Assim, pela definição de $p$ temos que $p: X_{1} \rightarrow X_{2}$ é um morfismo e $f_{1}=f_{2} \circ p$. De maneira análoga, podemos definir o morfismo $q: X_{2} \rightarrow X_{1}$ tal que $f_{2}=f_{1} \circ q$. Como as aplicações $p$ e $q$ são uma a inversa da outra, concluímos que $p$ é um isomorfismo.

4. Se $X$ é uma árvore, $X$ não possui circuitos. Logo, se $x$ é um vértice distinguido de $X$, teremos $\pi_{1}(X, x)=\{1\}$ e como $f_{*}$ é injetiva, segue que $f_{*}\left(\pi_{1}(X, x)\right)=\{1\}$. A recíproca é direta. Além disso, se $g \in \pi_{1}(Y, y)$ e $v \in V^{X}$ então $g v=v$ apenas se $g=1$, pois $X$ é árvore. Logo $\pi_{1}(Y, y)$ age livremente em $X$. 
Agora, podemos definir um isomorfismo entre $\pi_{1}(Y, y) \backslash X$ e $Y$. Seja $\pi_{1}(Y, y) \backslash X=$ $\left\{\pi_{1}(m) \mid m \in X\right\}$ o conjunto das órbitas dos elementos de $X$ e considere $f^{\prime}$ : $\pi_{1}(Y, y) \backslash X \rightarrow Y$ dada por $\pi_{1}(m) \mapsto f(m)$, onde $f$ é o recobrimento. Note que $f^{\prime}$ é um morfismo bijetivo. De fato, $f^{\prime}$ é sobrejetivo pois $f$ é recobrimento e $f^{\prime}$ restrita a estrela de $\pi_{1}(m)$ é injetiva, pois $f^{\prime}\left(\pi_{1}\left(e_{1}\right)\right)=f^{\prime}\left(\pi_{1}\left(e_{2}\right)\right) \Rightarrow f\left(e_{1}\right)=f\left(e_{2}\right) \Rightarrow e_{1}=e_{2}$.

5. Vamos assumir que $X$ é definido como na demonstração do item 2. Seja $G=$ $\pi_{1}(Y, y) / H$. Note que $G$ age à esquerda sobre $X$ de modo natural, ou seja, dado $(u, i)$ um vértice ou aresta do grafo $X$ e $H g$ uma classe lateral de $G$, temos $H g \cdot(u, i)=$ $(H g u, j)$. E, além disso, $H g$ envia $(u, i)$ para $(u, j)$ se $H g t_{i}=H t_{j}$.

Provaremos que $G$ age livremente em $X$. Suponha que exista $H g \in G$ qualquer e $(u, i) \in V^{X}$ tais que $H g \cdot(u, i)=(u, i)$. Mas, se isso acontece, $H g t_{i}=H t_{i} \Rightarrow g \in$ $H \Rightarrow H g=H$. Portanto, a ação é livre.

Note que o grafo quociente $G \backslash X$ é isomorfo a $Y$ pelo item 4.

Agora, usando recobrimento de grafos, é possível apresentar uma outra demonstração para o Teorema de Nielsen-Schereier sobre o subgrupo de um grupo livre.

Teorema 2.46. Qualquer subgrupo de um grupo livre é livre.

Demonstração. Seja $H$ um subgrupo do grupo livre $F$. Podemos identificar $F$ com o grupo $\pi_{1}(Y, y)$, onde $Y$ é um grafo com único vértice $y$. Pelo teorema anterior, existe um recobrimento $f:(X, x) \rightarrow(Y, y)$ tal que a aplicação injetiva $f_{*}$ identifica o grupo $\pi_{1}(X, x)$ com o subgrupo $H$, ou seja, $f_{*}\left(\pi_{1}(X, x)\right)=H$. Pelo Teorema 2.39 sabemos que $\pi_{1}(X, x)$ é livre e, portanto, $H$ é livre.

\subsection{Grafos de grupos}

Apresentaremos aqui apenas as definições básicas de grafo de grupos e de grupo fundamental de um grafo de grupos, juntamente com alguns exemplos. Para maior aprofundamento, veja as referências [1], [2], [6], [24]. 
Definição 2.47. Um grafo de grupos $(\mathbb{G}, Y)$ consiste de um grafo conexo $Y$, um grupo de vértices $G_{v}$ para cada $v \in V^{Y}$, um grupo de arestas $G_{e}$ para cada e $\in E^{Y}$ e monomorfismos $\left\{\alpha_{e}: G_{e} \rightarrow G_{\alpha(e)}\right\},\left\{\omega_{e}: G_{e} \rightarrow G_{\omega(e)}\right\}$, onde $\omega_{e}=\alpha_{\bar{e}}$ e tais que $G_{e}=G_{\bar{e}}$.

Dado qualquer grafo de grupos $(\mathbb{G}, Y)$, podemos associar a ele um grupo análogo ao grupo fundamental de um espaço topológico, denominado grupo fundamental e denotado por $\pi_{1}(\mathbb{G}, Y)$. Esse grupo $\pi_{1}(\mathbb{G}, Y)$ é construído de seus grupos de vértices e de seus grupos de arestas usando produto amalgamado e HNN extensões.

Para definir o grupo fundamental de um grafo de grupos $(\mathbb{G}, Y)$, vamos primeiro definir o grupo $F(\mathbb{G}, Y)$. Esse grupo será gerado pelos grupos $G_{v}$, onde $v \in V^{Y}$, e os elementos $t_{e}$, onde $e \in E^{Y}$, com as relações $t_{\bar{e}}=t_{e}^{-1}$ e $t_{e}^{-1} \alpha_{e}(g) t_{e}=\alpha_{\bar{e}}(g)$, se $g \in G_{e}$.

Mais precisamente, seja $\Gamma$ o produto livre de $G_{v}$ com o grupo $F$, onde $F$ é o grupo livre com base $\left\{t_{e} \mid e \in E^{Y}\right\}$. Considere também

$$
N:=\left\langle t_{\bar{e}} t_{e}, t_{e}^{-1} \alpha_{e}(g) t_{e} \alpha_{\bar{e}}(g)^{-1} \mid e \in E^{Y}, g \in G_{e}\right\rangle .
$$

Então, $F(\mathbb{G}, Y)$ é o grupo quociente $\Gamma / N$.

Podemos agora definir o grupo fundamental de um grafo de grupos $(\mathbb{G}, Y)$. Daremos a definição com respeito a um vértice e com respeito a uma subárvore maximal do grafo $Y$.

Definição 2.48. Seja $(\mathbb{G}, Y)$ um grafo de grupos e seja $P$ um vértice do grafo $Y$. $O$ grupo fundamental $\pi_{1}(\mathbb{G}, Y, P)$ do grafo de grupos $(\mathbb{G}, Y)$ com respeito ao vértice $P$ é o subgrupo do grupo $F(\mathbb{G}, Y)$ consistindo de todos os elementos da forma $g_{0} t_{e_{1}} g_{1} t_{e_{2}} \ldots t_{e_{n}} g_{n}$, onde $e_{1} e_{2} \ldots e_{n}$ é um caminho fechado em $Y$ com vértice inicial $P$, $g_{0} \in G_{P}, g_{i} \in G_{\omega\left(e_{i}\right)}, 1 \leq i \leq n$.

Definição 2.49. Seja $(\mathbb{G}, Y)$ um grafo de grupos e seja $T$ a subárvore maximal do grafo $Y$. O grupo fundamental $\pi_{1}(\mathbb{G}, Y, T)$ do grafo de grupos $(\mathbb{G}, Y)$ com respeito a subárvore $T$ é o grupo quociente do grupo $F(\mathbb{G}, Y)$ pelo fecho normal do conjunto de elementos $t_{e}$, onde $e \in E^{T}$.

Vejamos alguns exemplos:

Exemplo 2.50. 1. Se $G_{v}=\{1\}$ para todo vértice $v \in V^{Y}$, então $\pi_{1}(\mathbb{G}, Y, P) \cong$ $\pi_{1}(Y, P)$, que é o grupo fundamental do grafo $Y$ com respeito ao vértice $P$, como visto na Seção 2.3. 
2. Se $Y=\stackrel{P}{Q} \quad \stackrel{Q}{\bullet}$ é um segmento, então o grupo $\pi_{1}(\mathbb{G}, Y, Y)$ é isomorfo ao produto livre amalgamado de $G_{P}$ por $G_{Q}$ sobre os subgrupos amalgamados $\alpha_{e}\left(G_{e}\right)$ e $\alpha_{\bar{e}}\left(G_{e}\right)$.

3. Se $Y={ }^{P} \bigcirc e$ é um laço, então o grupo $\pi_{1}(\mathbb{G}, Y, P)$ é isomorfo a HNN extensão com base $G_{P}$ e os subgrupos associados $\alpha_{e}\left(G_{e}\right)$ e $\alpha_{\bar{e}}\left(G_{e}\right)$.

Agora, suponha que um grupo $G$ age sem inversão de arestas em uma árvore $X$. Então associamos a essa ação um grafo $(\mathbb{G}, Y)$ de grupos e o grafo $Y$ é o grafo quociente $G \backslash X$. Os grupos de vértices e os grupos de arestas do grafo de grupos $(\mathbb{G}, Y)$ são estabilizadores de um conjunto selecionado de arestas e vértices de $X$. Assim, se considerarmos o grupo $G$ agindo sem inversão de arestas na árvore $X$ e construírmos o correspondente grafo de grupos $(\mathbb{G}, Y)$, obtemos que

$$
G \cong \pi_{1}(\mathbb{G}, Y)
$$

Esse resultado é o conhecido Teorema de Estrutura para grupos agindo sem inversão de arestas em uma árvore e pode ser encontrado em Serre [24]. 


\section{Capítulo 3}

\section{A Conjectura de Hanna Neumann}

Nesse capítulo apresentaremos os resultados principais a respeito da Conjectura de Hanna Neumann. Iniciaremos apresentando o resultado demonstrado por Hanna Neumann em 1956, o qual foi baseado no trabalho de Howson e deu origem a sua famosa conjectura. Depois, apresentaremos uma outra demonstração para o resultado de Hanna Neumann, dada por seu filho Walter Neumann em 1990, o qual formulou a versão forte da conjectura. Na sequência, faremos a demonstração da conjectura a partir do trabalho desenvolvido por Mineyev e apresentaremos a versão dada por Dicks para a demonstração. Por fim, mostraremos que o limite proposto por Hanna Neumann para o posto da interseção de dois subgrupos finitamente gerados de um grupo livre é o melhor possível.

\subsection{A Conjectura}

Seja $F$ um grupo livre e sejam $A$ e $B$ dois subgrupos finitamente gerados de $F$. Howson, em 1954, mostrou em [11] que a interseção de $A$ e $B$ é finitamente gerada e obteve uma limitação superior para o posto dessa interseção. Se denotarmos os postos de $A, B$ e $A \cap B$ por $m, n$ e $\mathcal{N}$ respectivamente, obteve que $\mathcal{N}-1 \leq 2 m n-m-n$.

Hanna Neumann, em 1956, baseada no trabalho de Howson, apresentou uma pequena modificação da demonstração e mostrou que o limitante para o posto da interseção poderia ser melhorado. O limite superior que ela obteve, para $m \geq n$, foi:

$$
\mathcal{N}-1 \leq 2 m n-2 m-n
$$


Percebendo que poderia modificar alguns passos de sua demonstração, Hanna Neumann publicou, em 1957, um adendo em que melhorou o limite para

$$
\mathcal{N}-1 \leq 2(m-1)(n-1)
$$

e conjecturou que o fator 2 dessa desigualdade poderia ser removido, mais precisamente:

Conjectura de Hanna Neumann. Sejam A e B subgrupos finitamente gerados de um grupo livre $F$, de postos $m$ e $n$ respectivamente, e seja $\mathcal{N}$ o posto da interseção $A \cap B$. Então, $\mathcal{N}-1 \leq(m-1)(n-1)$.

Segundo a demonstração dada por Howson em seu artigo, vamos assumir, sem perda de generalidade, que o grupo livre F possui posto 2. Para apresentar a demonstração dada por Hanna Neumann em seus artigos [20] e [21], vamos introduzir alguns termos e definições.

Definição 3.1. Seja $S$ um subconjunto do grupo livre $F=F(x, y)$ gerado por $x$ e $y$. Dizemos que $S$ possui s finais se ele contém no máximo s elementos cujas representações como palavras reduzidas em $x$, y terminam em letras distintas $x, y, x^{-1}, y^{-1}$. Então $0 \leq$ $s \leq 4$ e $s=0$ somente se $S$ é vazio ou consiste do elemento unidade.

No grupo livre $F$, alguns elementos são chamados de pontos de ramificação para um subgrupo $A$ finitamente gerado de $F$ :

Definição 3.2. O elemento $f \in F$ é dito um ponto de ramificação com respeito a $A$ se existem elementos distintos $f_{1}, f_{2} \in F$ tais que:

i) $f f_{1}, f f_{2} \in A$, onde $f f_{1}, f f_{2} \neq 1$;

ii) $f$ é o encontro de $f f_{1}$ e $f f_{2}$.

Observação 3.3. Podemos ilustrar essa definição em um grafo:

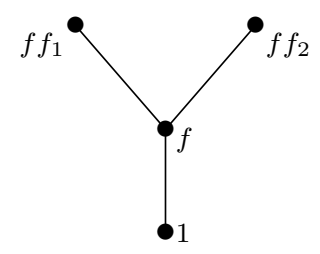


Além disso, iremos dizer que dois pontos de ramificação $f_{1}$ e $f_{2}$ com respeito a $A$ são equivalentes módulo $A$ se $f_{1} f_{2}^{-1} \in A$, isto é, se $f_{1}$ e $f_{2}$ pertencem a mesma classe lateral à direita de $A$ em $F$.

Modificando a definição dada inicialmente por Howson, Hanna Neumann caracterizou a ordem dos pontos de ramificação:

Definição 3.4. Seja $f \in F$. Definimos a ordem de $f$ como segue:

1) Se $f \notin A$, então:

i) fé um ponto de ramificação de ordem 2 se a classe Af possui 4 finais;

ii) $f$ é um ponto de ramificação de ordem 1 se a classe Af possui 3 finais;

iii) $f$ tem ordem 0 se a classe Af possui menos que 3 finais e, nesse caso, não é ponto de ramificação;

2) Se $f \in A$, então:

i) $f$ é um ponto de ramificação de ordem 3 se A possui 4 finais;

ii) $f$ é um ponto de ramificação de ordem 2 se A possui 3 finais;

iii) $f$ é um ponto de ramificação de ordem 1 se A possui 2 finais;

iv) $f$ tem ordem 0 se A possui menos que 2 finais e, nesse caso, não é ponto de ramificação;

Com essa definição, Howson mostrou que a soma das ordens de todos os pontos de ramificação tomados de um conjunto de representantes das classes laterais de $A$ em $F$ é $2 m-1$, onde $m$ é o posto de $A$, ou seja:

Lema 3.5 (Howson). Sejam $T$ um conjunto de representantes das classes laterais de A em $F$ e $f \in T$ um ponto de ramificação com respeito a $A$. Se denotarmos a ordem de $f$ por o $(f)$ e se $A$ possui posto finito $m$, temos:

$$
O(A):=\sum_{f \in T} o(f)=2 m-1
$$


Exemplo 3.6. Vamos considerar $S_{3}$ para exemplificar as definições vistas acima:

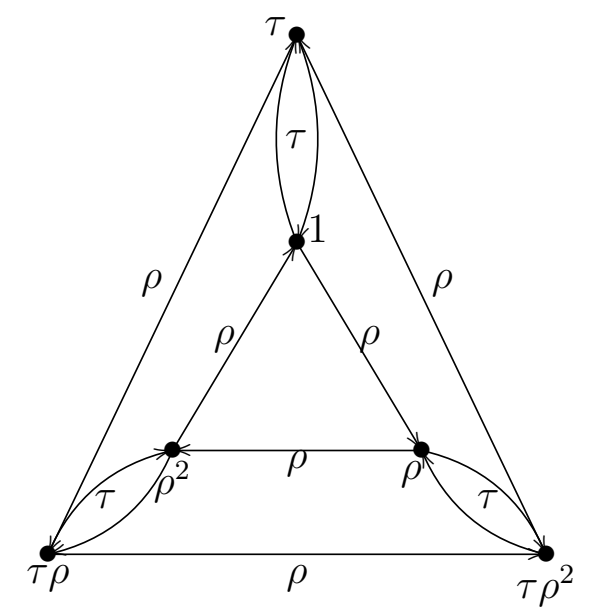

Considere o subgrupo $A=\langle\rho\rangle=\left\{1, \rho, \rho^{2}\right\}$ de $S_{3}$ e seja $T=\{1, \tau\}$. Note então que:

- $o(1)=1$, pois $1 \in A$ e a classe $A 1=\left\{1, \rho, \rho^{2}\right\}=\{1, \rho, \tau \rho \tau\}$ possui 2 finais;

- $o(\tau)=0$, pois $\tau \notin A$ e a classe $A \tau=\left\{\tau, \rho \tau, \rho^{2} \tau\right\}=\{\tau, \rho \tau, \tau \rho\}$ possui 2 finais, logo $\tau$ não é ponto de ramificação com respeito a $A$.

Assim, teremos

$$
o(1)+o(\tau)=1+0=1=2 \cdot 1-1
$$

estando de acordo com o Lema 3.5 enunciado acima.

Agora, podemos identificar a ordem da classe lateral $A f$ e do subgrupo $A$ a partir da definição dada anteriormente. A ordem da classe lateral Af seguirá do item 1) da Definição 3.4 e a ordem de $A$ seguirá de 2), porém diminuiremos essa ordem em uma unidade, ou seja, em detalhes teremos:

Definição 3.7. 1) A classe lateral Af é de ordem 2 se possui 4 finais e de ordem 1 se possui 3 finais, caso contrário é de ordem 0 ;

2) O subgrupo $A$ é de ordem 2 se possui 4 finais, de ordem 1 se possui 3 finais, de ordem 0 se possui 2 finais e de ordem -1 se possui 1 final.

Denotaremos por $o(A f)$ a ordem da classe lateral $A f$ e por $O(A)$ a soma das ordens de todas as classes laterais. Usando a definição acima, note que $O(A)$ será uma unidade menor que a ordem total obtida por Howson no Lema 3.5 para pontos de ramificação equivalentes módulo $A$, ou seja: 
Fato 1. Se A possui posto finito $m$, a ordem total de todas as classes laterais de $A$ em F é $O(A)=2 m-2$. E no caso de A possuir posto infinito, $O(A)$ é também infinito.

A partir dessas definições e resultados, mostraremos agora o teorema principal:

Teorema 3.8 (Hanna Neumann). Sejam $A$ e $B$ subgrupos finitamente gerados de um grupo livre $F$, de postos $m$ e $n$ respectivamente, e seja $\mathcal{N}$ o posto da interseção $N=A \cap B$. Então $\mathcal{N}-1 \leq 2(m-1)(n-1)$, onde $m \geq n$.

Demonstração. Para mostrar o limite para $\mathcal{N}$, podemos assumir primeiramente que pelo menos um dos subgrupos, $A$ ou $B$, possui ordem não negativa. De fato, suponha que $o(A)=-1$, então todos os elementos $\neq 1$ de $A$ possuem o mesmo final pela Definição 3.7. Seja $x^{-1}$ o maior segmento inicial comum de todas as palavras reduzidas que representam os elementos de $A$. Então, se $1 \neq a \in A$ temos $a=x^{-1} a_{1} x$, com $x \neq 1$ e $a_{1} \in A_{1} \subset A$. Assim, $A=x^{-1} A_{1} x$, onde $A_{1}$ é agora de ordem não negativa, pois se $o\left(A_{1}\right)=-1$, todo elemento de $A_{1}$ teria o mesmo final e este final poderia ser acrescentado à palavra $x$, o que estaria contadizendo o fato de $x$ ser maximal. Considere o automorfismo interno $\xi: F \rightarrow F$, dado por $\xi(F)=x F x^{-1}$. Então $\xi(A)=\xi\left(x^{-1} A_{1} x\right)=A_{1}$ e $\xi(B)=B_{1}$ e, assim, $\operatorname{rank}(A \cap B)=\operatorname{rank}\left(A_{1} \cap B_{1}\right)$, onde $A_{1}$ é de ordem não negativa. Portanto, podemos considerar pelo menos um dos subgrupos de ordem não negativa.

Como ambos os subgrupos possuem posto finito, vamos assumir que o grupo de menor posto possui ordem não negativa, ou seja, que o subgrupo $B$ de posto $n$ tem ordem não negativa. Então, todas as classes laterais de $A$ e $B$, exceto possivelmente $A$ mesmo, terão ordem não negativa.

Consideremos as classes laterais de $N=A \cap B$. Note que cada classe lateral $N h$ de $N$ em $F$ será unicamente a interseção $A f \cap B g$ para algum par de classes laterais de $A$ e $B$, respectivamente. Desse modo, a definição de ordem de uma classe lateral, dada anteriormente, nos fornece uma relação entre a ordem de uma classe lateral de $N$ e as ordens dos correspondentes pares de classes laterais de $A$ e $B$. Temos então dois casos a considerar.

$1^{\circ}$ caso: $\operatorname{Se} o(A) \geq 0$.

Se $N h=A f \cap B g$, então $o(N h) \leq \min \{o(A f), o(B g)\}$, onde $o(A f)$ e $o(B g)$ podem assumir os valores 0,1 ou 2 e a interseção é analisada através dos possíveis finais de $A f$ 
e $B g$. Note que somente finitas classes laterais de $N$ tem ordem positiva, já que somente finitos dos números $o(A f)$ e $o(B g)$ são positivos, pois $A$ e $B$ são subgrupos finitamente gerados. Temos então que:

1. $o(N h) \leq \frac{1}{2} o(A f) o(B g)$ se $\max \{o(A f), o(B g)\}=2$;

2. $o(N h) \leq o(A f) o(B g)$, se ambos $o(A f)$ e $o(B g)$ são menores que 2 .

Assim, usando uma estimativa aproximada, podemos assumir que $o(N h) \leq o(A f) o(B g)$ para todas classes laterais $N h$ de $N$ em $F$. Agora, somando sobre todos $N h$ obtemos:

$$
O(N)=\sum_{h} o(N h) \leq \sum_{f, g} o(A f) o(B g) \leq \sum_{f} o(A f) \sum_{g} o(B g)=O(A) O(B) .
$$

Então, pelo Fato 1 observado anteriormente, temos:

$$
2 \mathcal{N}-2 \leq(2 m-2)(2 n-2) \Rightarrow \mathcal{N}-1 \leq 2(m-1)(n-1)
$$

$2^{\circ}$ caso: Se $o(A)=-1$.

Nesse caso, $A$ possui somente um final, então ou $N$ é trivial ou é um subgrupo de $A$, também de ordem -1 . Portanto, se $N$ é não trivial, temos:

$$
\sum_{h \neq 1} o(N h)=2 \mathcal{N}-1 \text { e } \sum_{f \neq 1} o(A f)=2 m-1 .
$$

As desigualdades 1 e 2 do caso anterior continuam valendo se $A f \neq A$, mas as interseções $A \cap B g$, onde $B g \neq B$, não contribuem para a ordem total de $N$, pois elas possuem somente um final como $A$. Então:

$$
\begin{aligned}
& \sum_{h \neq 1} o(N h) \leq \sum_{f \neq 1, g} o(A f) o(B g) \leq \sum_{f \neq 1} o(A f) \sum_{g} o(B g) . \\
\Rightarrow & 2 \mathcal{N}-1 \leq(2 m-1)(2 n-2) \Rightarrow \mathcal{N}-1 \leq 2 m n-2 m-n .
\end{aligned}
$$

Agora, note que se $B$ é cíclico, temos $n=1$, então a desigualdade (3.1) nos dá $\mathcal{N} \leq 1$ e a desigualdade (3.2) nos dá $\mathcal{N}=0$, que é o limite correto em ambos os casos. Se $n=2$, as duas desigualdades nos fornecem $\mathcal{N} \leq 2 m-1$. Como em todos os outros casos a desigualdade (3.2) é mais fraca que a desigualdade (3.1), valeria que $\mathcal{N}-1 \leq 2 m n-2 m-n$.

Porém, segundo o adendo publicado por Hanna Neumann em 1957, o automorfismo interno $\xi$ considerado no começo da demonstração, pode ser escolhido para todo o grupo, 
assim a interseção $N=A \cap B$ pode ser transformada em um grupo $N_{1}=A_{1} \cap B_{1}$ de ordem não negativa, onde $A_{1}$ e $B_{1}$ também possuem ordem não negativa. Desse modo, ambos os subgrupos $A$ e $B$ podem ser considerados de ordem não negativa e, assim, somente o $1^{\mathrm{o}}$ caso acima precisa ser considerado. Portanto, vale a desigualdade que queríamos:

$$
\mathcal{N}-1 \leq 2(m-1)(n-1)
$$

Observação 3.9. Hanna Neumann afirmou em seu adendo que seria possível considerar esse automorfismo para a interseção $N=A \cap B$, mas não descreveu de que forma. Além disso, ela agradeceu ao Prof. R. Baer, responsável por fazer essa observação.

\subsection{Versão forte da Conjectura}

Em 1990, Walter Neumann, filho de Hanna Neumann, formulou a versão forte da conjectura. Dados $A$ e $B$ subgrupos finitamente gerados de um grupo livre $F$ e $N=A \cap B$, Walter questionou-se sobre o cálculo do posto da interseção quando $N$ percorre um conjunto de representantes de classes de conjugação de interseções não triviais $N=y^{-1} A y \cap z^{-1} B z$, e mostrou que o limitante para esse caso continua o mesmo. Para isso, ele reescreveu o posto de um grupo livre, definindo o posto reduzido, como veremos adiante.

Mas, primeiro, apresentaremos algumas definições necessárias para demonstrar o teorema de Hanna Neumann de outra maneira que a apresentada anteriormente, e depois provar a versão forte do resultado. Ambas demonstrações são devidas a Walter Neumann (veja em [22]).

Definição 3.10. Um ramo maximal de um grafo $X$ é o mais longo subgrafo contrátil de $X$ que encontra o resto do grafo somente no final de uma aresta.

Agora, se cortarmos todos os ramos maximais de $X$, obtemos a retração minimal $X_{0}$ de $X$, também chamada de espinha de $X$. Vamos definir formalmente a aplicação retração minimal:

Definição 3.11. A aplicação retraçãoo $r: X \rightarrow X_{0}$ é uma aplicação contínua homotópica à aplicação identidade em $X_{0}$ e tal que existe $h:[0,1] \times X \rightarrow X$ que satisfaz as condições: 
a) para todos os vértices $w$ em $X_{0}, r(w)=w$;

b) para todos os vértices $v \in X, h(0, v)=v$ e $h(0,1)=r(v)$;

c) para todos os vértices $v \in X_{0}, h(t, v)=v, \forall t \in[0,1]$.

A aplicação retração induz um isomorfismo entre o grupo fundamental do grafo e o grupo fundamental da espinha do grafo:

Teorema 3.12. A retração $r: X \rightarrow X_{0}$ induz um isomorfismo $r_{*}: \pi_{1}(X) \rightarrow \pi_{1}\left(X_{0}\right)$.

Demonstração. Para mostrar que $r_{*}$ é um homomorfismo, note que por $r$ ser uma aplicação contínua, caminhos fechados em $X$ são levados em caminhos fechados em $X_{0}$. Além disso, a aplicação leva caminhos homotópicos equivalentes em caminhos homotópicos equivalentes, pois para qualquer homotopia $h, r \circ h$ ainda é uma homotopia. Então, pela condição $a)$ da definição anterior, dados $x, y \in X_{0}$, temos $r(x y)=x y=r(x) r(y)$ e $r\left(x^{-1}\right)=x^{-1}=r(x)^{-1}$. A condição $\left.a\right)$ também implica que $r_{*}$ é sobrejetiva, pois os caminhos fechados em $X$ serão levados neles mesmos em $X_{0}$.

Resta mostrar que $r_{*}$ é injetiva. Para isso, considere um caminho fechado $p$ em $X$ com vértice base $w$, onde $w \in X_{0}$. Pelo item $\left.c\right), h(t, w)=w, \forall t \in[0,1]$, então $h(0, w)=w$ e $h(1, w)=w=r(w)$. Assim, $r(p)$ terá vértice base $w$ e, portanto, $r(p)$ e $p$ são caminhos homotópicos. Logo, $p$ e $r(p)$ estão na mesma classe de equivalência em $\pi_{1}(X)$ e $\pi_{1}\left(X_{0}\right)$. Portanto, $r_{*}$ é injetiva e, então, um isomorfismo.

Corolário 3.13. Se $\pi_{1}(X)$ é um grupo livre finitamente gerado, então a espinha $X_{0}$ de $X$ é um grafo finito.

Demonstração. Suponha que $X_{0}$ seja infinito. Então, devemos ter infinitos caminhos fechados, pois todas as árvores infinitas foram cortadas. Mas, nesse caso, $\pi_{1}\left(X_{0}\right)$ é infinito, o que gera uma contradição, pois pelo teorema anterior $\pi_{1}\left(X_{0}\right)$ é isomorfo a $\pi_{1}(X)$ que é finitamente gerado. Portanto, $X_{0}$ é finito.

O próximo teorema nos dá uma relação entre a característica de Euler de um grafo finito e o posto do grupo fundamental do grafo: 
Teorema 3.14. Sejam $\chi(X)=\left|V^{X}\right|-\left|E^{X}\right|$ a característica de Euler de um grafo finito e $\delta(v)$ a valência de um vértice $v \in V^{X}$. Se X é um grafo finito, então:

$$
2\left(\operatorname{rank}\left(\pi_{1}(X)\right)-1\right)=2(-\chi(X))=\sum_{v \in V^{X}}(\delta(v)-2) .
$$

Demonstração. Primeiro, vamos mostrar a igualdade da esquerda. Se $T$ é uma árvore de $X$, vamos contar seu número de vértices. Tomando um vértice inicial, cada aresta subsequente vai para outro vértice, e tanto o número de arestas como o de vértices aumenta de uma unidade. Calculando a característica de Euler de $T$, toda aresta e vértice são cancelados, exceto o primeiro vértice, ou seja, $\chi(X)=1+(1-1)+(1-1)+\cdots=1$.

Agora, considere que o grafo $X$ tem $k$ caminhos fechados e uma árvore maximal $X_{T}$. Note que cada caminho fechado pode ser pensado como uma árvore com uma aresta adicional fechando o caminho. Então,

$$
\chi(X)=\left|V^{X}\right|-\left|E^{X}\right|=\left|V^{X_{T}}\right|-\left(\left|E^{X_{T}}\right|+k\right)=1-k .
$$

Note que $k=\operatorname{rank}\left(\pi_{1}(X)\right)$ pela construção dos geradores do grupo fundamental, vista no Teorema 2.39. Então, $\chi(X)=1-\operatorname{rank}\left(\pi_{1}(X)\right)$, ou ainda, $-\chi(X)=\operatorname{rank}\left(\pi_{1}(X)\right)-1$, o que prova a primeira igualdade.

Para a segunda igualdade, precisamos notar que a soma das valências de todos os vértices do grafo $X$ é igual ao dobro do número de arestas de $X$, ou seja,

$$
\sum_{v \in V^{X}} \delta(v)=2\left|E^{X}\right|
$$

Isso ocorre pelo fato de que cada aresta incidente é contada duas vezes na soma da valência dos vértices. Assim, teremos:

$$
\begin{aligned}
2(-\chi(X)) & =2\left(\left|E^{X}\right|-\left|V^{X}\right|\right) \\
& =2\left|E^{X}\right|-2\left|V^{X}\right| \\
& =\sum_{v \in V^{X}} \delta(v)-2\left|V^{X}\right| \\
& =\sum_{v \in V^{X}}(\delta(v)-2) .
\end{aligned}
$$

Portanto, a igualdade do lado direito também é válida. 
Observação 3.15. A segunda igualdade no teorema só é válida quando $X$ é finito. No caso de $X$ possuir infinitos vértices, a soma será infinita ou zero, e não será necessariamente equivalente à característica de Euler. Por exemplo, considere uma árvore infinita unidimensional que possui característica de Euler 1, então teremos:

$$
\sum_{v \in V^{X}}(\delta(v)-2)=\sum_{v \in V^{X}} 2-2=0 \neq 1
$$

Agora, vamos redefinir o posto de um grupo livre, o qual será chamado de posto reduzido. Dado $F$ um grupo livre e $H$ um subgrupo finitamente gerado de $F$, escreveremos

$$
\bar{r}(H)=\max \{0, \operatorname{rank}(H)-1\}
$$

para denotar o posto reduzido do subgrupo $H$.

Com essa definição, o teorema de Hanna Neumann pode ser reescrito, como veremos abaixo. E a partir das definições e resultados provados acima, uma outra demonstração para o resultado é possível.

Teorema 3.16. Sejam $A$ e $B$ subgrupos finitamente gerados do grupo livre $F$ e $N=A \cap B$ não trivial. Então, $\bar{r}(N) \leq 2 \bar{r}(A) \bar{r}(B)$.

Demonstração. Podemos assumir, sem perda de generalidade, que $F$ tem posto 2 , gerado por elementos $a$ e $b$. Seja $X$ o buquê de 2 laços, cujo grupo fundamental é $F$.

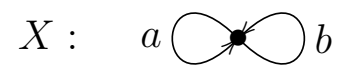

Para $H \leq F$ seja $X(H)$ um recobrimento de $X$, tal que $\pi_{1}(X(H))=H$, o que é possível pelo Teorema 2.45. Podemos considerar $X(H)$ o grafo de Schreier de $H$, como na Definição 2.34, ou seja, onde identificamos o conjunto de vértices de $X(H)$ com o conjunto $H \backslash F$ de classes laterais à direita de $H$ em $F$, onde as arestas marcadas por $a$ são os pares $(H x, H x a)$ e as marcadas por $b$ são os pares $(H x, H x b)$.

Assumindo que $H$ é não trivial, seja $X_{0}(H)$ a espinha de $X(H)$, que é um grafo homotopicamente equivalente a $X(H)$. Pelo Teorema 3.26, como $\pi_{1}(X(H))$ e $\pi_{1}\left(X_{0}(H)\right)$ são isomorfos, eles possuem o mesmo posto. Então, podemos usar o grafo $X_{0}(H)$ para descobrir sobre o posto de $H$, o que é mais conveniente. Se $H$ tem posto finito, então 
$X_{0}(H)$ é um grafo finito pelo Corolário 3.13 e, então, podemos usar o Teorema 3.14 sobre a característica de Euler:

$$
\begin{aligned}
2\left(\operatorname{rank}\left(\pi_{1}\left(X_{0}(H)\right)-1\right)\right. & =\sum_{v \in V^{X_{0}(H)}}(\delta(v)-2) \\
2 \bar{r}(H) & =\sum_{v \in V^{X_{0}(H)}}(\delta(v)-2) .
\end{aligned}
$$

Agora, sendo $N=A \cap B, N$ é subgrupo de $A$ e de $B$, então $X(N)$ é um grafo de recobrimento de $X(A)$ e de $X(B)$. Assim, as aplicações projeção $X(N) \rightarrow X(A)$ e $X(N) \rightarrow X(B)$ levam a espinha $X_{0}(N)$ nas espinhas $X_{0}(A)$ e $X_{0}(B)$, respectivamente. Então, existem duas aplicações injetivas $\lambda_{A}: V^{X_{0}(N)} \rightarrow V^{X_{0}(A)}$ e $\lambda_{B}: V^{X_{0}(N)} \rightarrow V^{X_{0}(B)}$, pela Definição 2.42 de recobrimento. Note que a injetividade também é válida para

$$
\lambda=\left(\lambda_{A}, \lambda_{B}\right): V^{X_{0}(N)} \rightarrow V^{X_{0}(A)} \times V^{X_{0}(B)} .
$$

Então, para $v \in V^{X_{0}(N)}$, temos que

$$
\begin{aligned}
0 \leq \delta(v)-2 & \leq \min \left\{\delta\left(\lambda_{A}(v)\right)-2, \delta\left(\lambda_{B}(v)\right)-2\right\} \\
& \leq\left(\delta\left(\lambda_{A}(v)\right)-2\right)\left(\delta\left(\lambda_{B}(v)\right)-2\right)
\end{aligned}
$$

Note que para $v \in V^{X_{0}(N)}$, temos $\delta\left(\lambda_{A}(v)\right)=\delta(v)$, pois recobrimentos são localmente homeomorfos para cada vértice. Então, pela injetividade da aplicação $\lambda$ e por (3.3) e (3.4), temos:

$$
\begin{aligned}
2 \bar{r}(N) & =\sum_{v \in V^{X_{0}(N)}}(\delta(v)-2) \\
& \leq \sum_{v \in V^{X_{0}(N)}}\left(\delta\left(\lambda_{A}(v)\right)-2\right)\left(\delta\left(\lambda_{B}(v)\right)-2\right) \\
& \leq \sum_{(a, b) \in V^{X_{0}(A)} \times V^{X_{0}}(B)}(\delta(a)-2)(\delta(b)-2) \\
& =\left(\sum_{a \in V^{X_{0}(A)}}(\delta(a)-2)\right)\left(\sum_{b \in V^{X_{0}(B)}}(\delta(b)-2)\right) \\
& =2 \bar{r}(A) 2 \bar{r}(B)=4 \bar{r}(A) \bar{r}(B)
\end{aligned}
$$

Portanto, $\bar{r}(N) \leq 2 \bar{r}(A) \bar{r}(B)$.

A partir dessa demonstração, Walter Neumann questionou se seria possível pensar no posto das interseções da forma $y^{-1} A y \cap z^{-1} B z$ além do posto da interseção $A \cap B$. Após 
fazer essa análise, ele reformulou a conjectura antes proposta por Hanna Neumann, que ficou conhecida como SHNC - Strengthened Hanna Neumann Conjecture. Descreveremos na sequência as ideias desenvolvidas por ele.

Seja $F$ um grupo livre, dados $x, y, z \in F$, as interseções do tipo $y^{-1} A y \cap z^{-1} B z$ são conjugadas a uma da forma $x^{-1} A x \cap B$. Além disso, se $y$ está na classe lateral dupla $A x B$, então $x^{-1} A x \cap B$ e $y^{-1} A y \cap B$ são conjugados. De fato, se $y=a x b$, teremos

$$
y^{-1} A y=b^{-1} x^{-1} a^{-1} A a x b=b^{-1} x^{-1} A x b=\left(x^{-1} A x\right)^{b} .
$$

Logo, $y^{-1} A y \cap B=\left(x^{-1} A x\right)^{b} \cap B$. Dessa forma, precisamos apenas considerar $x$ percorrendo um conjunto $S$ de classes laterais duplas representativas para $A \backslash F / B$. Vamos considerar então $S$ um subconjunto de $F$ tal que a aplicação $S \rightarrow A \backslash F / B$, dada por $s \mapsto A s B$, é bijetiva. Além disso, dado $x \in S$, vamos considerar $x^{-1} A x \cap B=A^{x} \cap B$ não trivial.

Seja então $\bar{r}(A, B):=\sum_{x \in S} \bar{r}\left(A^{x} \cap B\right)$. Note que o tamanho de $S$ e de $\bar{r}(A, B)$ depende somente das classes de conjugação de $A$ e $B$. Dessa forma, a conjectura de Hanna Neumann será fortalecida a partir da proposição abaixo.

Proposição 3.17. O conjunto $S$ é finito e $\bar{r}(A, B) \leq 2 \bar{r}(A) \bar{r}(B)$.

Demonstração. Podemos assumir, sem perda de generalidade, que $F$ tem posto 2, pois se $F$ fosse um grupo livre de posto maior, apenas o tamanho de $S$ e de $\bar{r}(A, B)$ poderiam aumentar.

Sejam $X(A)$ e $X(B)$ grafos considerados como no Teorema 3.16, ou seja, grafos de recobrimento do grafo $X$ (cujo grupo fundamental é $F$ ). Considere $X(A) \times X(B)$ o grafo com conjunto de vértices $V^{X(A)} \times V^{X(B)}$. Uma aresta nesse grafo terá, por exemplo, uma aresta com a marca $a$ do vértice $(u, v)$ para $\left(u^{\prime}, v^{\prime}\right)$ se, e somente se, $X(A)$ tem uma $a$-aresta de $u$ para $u^{\prime}$ e $X(B)$ tem uma $a$-aresta de $v$ para $v^{\prime}$.

Note que as componentes do grafo $X(A) \times X(B)$ são exatamente os grafos $X(N)$, onde $N$ percorre os grupos $x^{-1} A x \cap B$, com $x \in S$. De fato, considerando o grafo de Schreier definido anteriormente, podemos identificar os vértices dos grafos $X(A)$ e $X(B)$ com as classes laterais de $A$ e $B$ em $F$, respectivamente. Assim, considerando o grafo $X(A) \times X(B)$, se $K$ é uma componente conexa que contém o vértice $(A y, B z)$, então ela 
contém também o vértice $\left(A y z^{-1}, B\right)$. Portanto, cada componente contém um vértice da forma $(A x, B)$. O grupo fundamental dessa componente consiste de todos $z \in F$ tal que $A x z=A x$ e $B z=B$, isto é, $z \in x^{-1} A x$ e $z \in B$, ou seja, $z \in x^{-1} A x \cap B$. Além disso, outro vértice $(A y, B)$ estará na mesma componente se, e somente se, existe $z \in F$ com $A y=A x z$ e $B=B z$, isto é, para algum $z \in B$ temos $y \in A x B$.

Com isso, se denotarmos por $X_{0}(A, B)$ a união disjunta das espinhas $X_{0}(N)$, onde $N$ varia ao longo das interseções $x^{-1} A x \cap B$, com $x \in S$, então $X_{0}(A, B)$ é a união disjunta das componentes não contráteis de $X(A) \times X(B)$, ou seja, é um subgrafo de $X_{0}(A) \times X_{0}(B)$. Em particular, $X_{0}(A, B)$ é um grafo finito pelo Corolário 3.13 e então $S$ é finito. Também, pela equação (3.3) e usando o mesmo argumento do Teorema 3.16 teremos:

$$
\begin{aligned}
2 \bar{r}(A, B) & =\sum_{v \in V^{X_{0}(A, B)}}(\delta(v)-2) \\
& \leq \sum_{v \in V^{X_{0}(A, B)}}\left(\delta\left(\lambda_{A}(v)\right)-2\right)\left(\delta\left(\lambda_{B}(v)\right)-2\right) \\
& \leq \sum_{(a, b) \in V^{X_{0}(A)} \times V^{X_{0}(B)}}(\delta(a)-2)(\delta(b)-2) \\
& =\left(\sum_{a \in V^{X_{0}(A)}}(\delta(a)-2)\right)\left(\sum_{b \in V^{X_{0}(B)}}(\delta(b)-2)\right) \\
& =2 \bar{r}(A) 2 \bar{r}(B)
\end{aligned}
$$

Logo, $\bar{r}(A, B) \leq 2 \bar{r}(A) \bar{r}(B)$ como queríamos.

Agora, surge a seguinte questão:

$$
\bar{r}(A, B) \leq \bar{r}(A) \bar{r}(B) ?
$$

Essa desigualdade ficou conhecida como SHNC - Strengthened Hanna Neumann Conjecture, ou seja, a versão forte da Conjectura de Hanna Neumann. A partir disso, vários matemáticos passaram a tentar mostrar em seus trabalhos que a versão forte da conjectura era válida.

Observação 3.18. Note que, provando a versão forte da conjectura de Hanna Neumann, a primeira versão da conjectura está demonstrada em particular. 


\subsection{Demonstração da Conjectura}

Apresentaremos agora a demonstração da versão forte da conjectura dada por Igor Mineyev em [18]. Essa demonstração foi a segunda apresentada por Mineyev, sendo baseada nas contribuições dadas por Warren Dicks à sua primeira demonstração (veja em [17]).

Para isso, inicialmente daremos algumas definições que serão necessárias na construção da demonstração.

Definição 3.19. O posto reduzido de um grafo finito $Y$ é o número

$$
\bar{r}(Y):=\sum_{K \in \operatorname{Comp}(Y)} \max \{0,-\chi(K)\},
$$

onde $K \in \operatorname{Comp}(Y)$ é uma componente conexa do grafo $Y$, $\chi$ é a característica de Euler $e \bar{r}(\emptyset)=0$.

Observação 3.20. Essa definição pode ser vista de outra maneira pelo Teorema 3.14:

$$
\bar{r}(Y)=\sum_{K \in \operatorname{Comp}(Y)} \max \left\{0, \operatorname{rank}\left(\pi_{1}(K)\right)-1\right\}=\sum_{K \in \operatorname{Comp}(Y)} \bar{r}\left(\pi_{1}(K)\right)
$$

Definiremos agora os elementos necessários para a apresentação da demonstração de Mineyev que são: árvore, floresta, flor e jardim. Em seguida, iremos definir conjuntos essenciais e conjuntos essenciais maximais.

Definição 3.21. Uma árvore é um grafo contrátil, isto é, homotopicamente equivalente a um ponto. Uma floresta é a união disjunta de árvores. Uma flor é um grafo homotopicamente equivalente a um círculo. Um jardim é a união disjunta de árvores e flores.

Note que o posto reduzido de cada elemento definido acima é nulo. De fato, a característica de Euler de uma árvore é sempre igual a 1 e de uma flor é igual a zero, implicando que seus postos reduzidos sejam nulos. Como uma floresta é a união disjunta de árvores e um jardim é a união disjunta de árvores e flores, o resultado segue imediato.
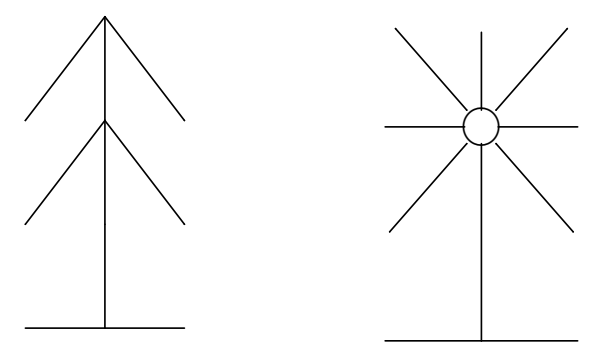
Definição 3.22. Uma aresta $\sigma$ em um grafo finito $Y$ é chamada essencial em $Y$ se $\bar{r}(Y \backslash$ $\{\sigma\})=\bar{r}(Y)-1$, ou seja, removendo uma aresta, o posto reduzido decresce exatamente uma unidade. De modo geral, um conjunto de arestas $E \subseteq E^{Y}$ é dito um conjunto essencial em $Y$ se $\bar{r}(Y \backslash E)=\bar{r}(Y)-\# E$.

Dada a definição acima, quando uma aresta não for essencial, diremos que ela é inessencial. O mesmo vale para conjuntos de arestas.

Definição 3.23. Um conjunto essencial maximal é um conjunto essencial que é maximal com respeito à inclusão.

Podemos dar uma caracterização para conjuntos essenciais e conjuntos esenciais maximais. Um conjunto $E \subseteq E^{Y}$ é essencial em $Y$ se para cada $\sigma \in E$, a componente de $Y \backslash E$ contendo $\alpha(\sigma)$ e a componente contendo $\omega(\sigma)$ não forem árvores.

De fato, seja $\sigma \in E \subseteq E^{Y}$ e suponha que ambas as componentes de $Y \backslash E$ contendo $\alpha(\sigma)$ e $\omega(\sigma)$ sejam árvores. Nesse caso, teríamos $\bar{r}(Y \backslash\{\sigma\})=0$ e $\bar{r}(Y)-1=-1$, pois o posto reduzido de uma árvore é nulo, $\operatorname{logo} E$ não seria um conjunto essencial. No caso em que uma das componentes é uma árvore, encontramos do mesmo modo que $\bar{r}(Y \backslash\{\sigma\}) \neq \bar{r}(Y)-1$. Agora, suponha que a componente $Y_{1}$ contendo $\alpha(\sigma)$ e a componente $Y_{2}$ contendo $\omega(\sigma)$ não sejam árvores, se $Y_{1}$ contém $k$ caminhos fechados e $Y_{2}$ contém $k^{\prime}$ caminhos fechados, teremos:

$$
\bar{r}(Y \backslash\{\sigma\})=(k-1)+\left(k^{\prime}-1\right)=\left(\left(k+k^{\prime}\right)-1\right)-1=\bar{r}(Y)-1 .
$$

Ou seja, como podemos fazer isso para cada $\sigma \in E$, o conjunto $E \subseteq E^{Y}$ é essencial em $Y$.

Além disso, um conjunto $E \subseteq E^{Y}$ é essencial maximal se, e somente se, $\bar{r}(Y \backslash E)=$ $\bar{r}(Y)-\# E=0$. De fato, através da remoção das arestas de $E$, uma por uma, observa-se que o posto reduzido do grafo $Y$ decresce exatamente uma unidade a cada remoção e como esse conjunto é maximal, no final do processo, quando todas as arestas tiverem sido removidas, esse número chega a zero.

\subsubsection{Construção de folhagens e sistemas}

A partir de agora, vamos considerar $\Gamma$ um grupo livre e $A$ e $B$ subgrupos finitamente gerados de $\Gamma$. Seja $\hat{X}$ o grafo de Cayley de $\Gamma$ com respeito a alguma base, então $\hat{X}$ é uma 
árvore pela Proposição 2.32. A partir de $A$ e $B$ vamos definir dois novos conjuntos $\mathcal{A}$ e $\mathcal{B}$. Vamos descrever a construção de $\mathcal{A}$, e a de $\mathcal{B}$ será análoga:

1. Se $A$ é trivial, seja $\mathcal{A}$ o conjunto vazio;

2. Se $A$ é não trivial, seja $\mathcal{A}$ uma subárvore de $\Gamma$ invariante pela ação de $A$ e tal que a $A$-ação em $\mathcal{A}$ é cocompacta, isto é, o grafo quociente $A \backslash \mathcal{A}$ é finito. Essa árvore pode ser construída escolhendo um ponto base $y \in V^{\hat{X}}$ e a partir dele construindo um caminho para ay, para cada a pertencente ao conjunto de geradores finitos de $A$. Então, $\mathcal{A}$ será a união de todas as translações desses caminhos.

A partir de translações de $\mathcal{A}$ em $\hat{X}$, vamos definir o grafo induzido por $A$. Seja $y \in \Gamma / A$ uma classe lateral à esquerda de $A$ em $\Gamma$, então $y \mathcal{A}$ será o subgrafo $\bar{y} \mathcal{A} \subseteq \hat{X}$, onde $\bar{y}$ é qualquer representante da classe lateral à esquerda de $y$.

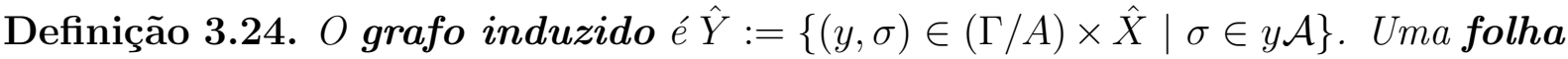
no grafo $\hat{Y}$ para cada $y \in \Gamma / A$ é o grafo $\hat{Y}_{y}:=\{(y, \sigma) \mid \sigma \in y \mathcal{A}\}$.

Note que o grafo $\hat{Y}_{y} \subseteq \hat{Y}$ e $\hat{Y}$ será a união disjunta de suas folhas, isto é, $\hat{Y}=\bigcup_{y \in \Gamma / A} \hat{Y}_{y}$. Denotaremos por $\mathcal{F}(\hat{Y})$ o conjunto de folhas de $\hat{Y}$.

Outra definição importante é a de folhagem, que será utilizada durante toda a construção da demonstração da conjectura.

Definição 3.25. Chamaremos de folhagem uma aplicação entre grafos cuja restrição a cada componente conexa do domínio é injetiva.

Assim, podemos definir a aplicação folhagem entre o grafo induzido e grafo de Cayley de $\Gamma$ :

$$
\hat{\alpha}: \hat{Y} \rightarrow \hat{X}, \text { dada por } \hat{\alpha}(y, \sigma):=\sigma \text {. }
$$

Vamos considerar $\Gamma$ agindo em $\hat{Y}$ de maneira natural, ou seja, para $g \in \Gamma,(y, \sigma) \in \hat{Y}$ temos:

$$
g \cdot(y, \sigma):=(g y, g \sigma)
$$

Essa $\Gamma$-ação tem algumas propriedades importantes: 
i) A $\Gamma$-ação comuta com a folhagem $\hat{\alpha}$ e, por isso, $\hat{\alpha}$ é chamada de $\Gamma$-folhagem.

De fato, por um lado temos

$$
g \cdot(\hat{\alpha}(y, \sigma))=g \cdot \sigma=g \sigma
$$

e, por outro

$$
\hat{\alpha}(g \cdot(y, \sigma))=\hat{\alpha}((g y, g \sigma))=g \sigma .
$$

ii) A $\Gamma$-ação é livre, pois para $1 \neq g \in \Gamma$ temos

$$
g \cdot(y, \sigma)=(g y, g \sigma) \neq(y, \sigma)
$$

E ela age em $\mathcal{F}(\hat{Y})$ permutando as folhas de $\hat{Y}$, de forma que $g \cdot \hat{Y}_{y}=\hat{Y}_{g y}$.

iii) Os estabilizadores das folhas de $\hat{Y}$ são conjugados de $A$.

De fato, eles pertencerão ao conjunto $\operatorname{Stab}_{\Gamma}\left(\hat{Y}_{y}\right)=\left\{\gamma \in \Gamma \mid \gamma \cdot \hat{Y}_{y}=\hat{Y}_{y}\right\}$ e então, dado $y=\bar{y} A \in \Gamma / A$, temos:

$$
\begin{aligned}
\gamma \in \operatorname{Stab}_{\Gamma}\left(\hat{Y}_{y}\right) & \Leftrightarrow(\gamma y, \gamma \sigma)=(y, \sigma) \\
& \Leftrightarrow \gamma y=y \\
& \Leftrightarrow \gamma \bar{y} A=\bar{y} A \\
& \Leftrightarrow \gamma \bar{y} A \bar{y}^{-1}=\bar{y} A \bar{y}^{-1} \\
& \Leftrightarrow \gamma \in A^{\bar{y}^{-1}} .
\end{aligned}
$$

Portanto, $\operatorname{Stab}_{\Gamma}\left(\hat{Y}_{y}\right)=A^{\bar{y}^{-1}}=A^{y^{-1}}$, para alguma escolha de $\bar{y} \in y$.

iv) Podemos identificar $\mathcal{A}$ com a folha $\hat{Y}_{1 A}=\{(1 A, \sigma) \mid \sigma \in \mathcal{A}\} \subseteq \hat{Y}$ pela inclusão $\mathcal{A} \hookrightarrow \hat{Y}$, dada por $\sigma \mapsto(1 A, \sigma)$.

Como dito anteriormente, a construção do conjunto $\mathcal{B}$ é similar e não será tão detalhada. Para o subgrupo $B \leq \Gamma$ podemos definir a subárvore $B$-invariante $\mathcal{B} \subseteq \hat{X}$. Assim, para $z \in \Gamma / B$, classe lateral à esquerda de $B$ em $\Gamma, z \mathcal{B}$ é o subgrafo $\bar{z} \mathcal{B} \subseteq \hat{X}$, para algum representante $\bar{z}$ da classe lateral de $z$. O grafo induzido será:

$$
\hat{Z}:=\{(z, \sigma) \in(\Gamma / B) \times \hat{X} \mid \sigma \in z \mathcal{B}\}
$$


Para $z \in \Gamma / B, \hat{Z}_{z}:=\{(z, \sigma) \mid \sigma \in z \mathcal{B}\}$ é uma folha em $\hat{Z}$ e $\hat{\beta}: \hat{Z} \rightarrow \hat{X}$ é a $\Gamma$-folhagem através da $\Gamma$-ação em $\hat{Z}$. Além disso, as demais propriedades i), ii), iii) e iv) da $\Gamma$-ação descritas acima são válidas para $\mathcal{B}$. Em particular, teremos $\operatorname{Stab}_{\Gamma}\left(\hat{Z}_{z}\right)=B^{z^{-1}}$ e o grafo $\mathcal{B}$ pode ser identificado com a folha $\hat{Z}_{1 B}$ de $\hat{Z}$.

Observação 3.26. Como A é subgrupo de $\Gamma$ e $\hat{Y}_{1 A}$ é uma folha de $\hat{Y}$, o grafo quociente $Y:=\Gamma \backslash \hat{Y}$ é isomorfo ao grafo $A \backslash \hat{Y}_{1 A}=A \backslash \mathcal{A}$.

Da mesma forma, o grafo quociente $Z:=\Gamma \backslash \hat{Z}$ é isomorfo ao grafo $B \backslash \hat{Z}_{1 B}=B \backslash \mathcal{B}$.

Agora, vamos definir o produto fibrado $\hat{S}$ entre os grafos induzidos $\hat{Y}$ e $\hat{Z}$ sobre o grafo de Cayley $\hat{X}$ de $\Gamma$.

Definição 3.27. O produto fibrado $\hat{S}$ de $\hat{Y}$ e $\hat{Z}$ sobre $\hat{X}$ é o conjunto

$$
\hat{S}:=\{(y, z, \sigma) \mid(y, \sigma) \in \hat{Y},(z, \sigma) \in \hat{Z}\}
$$

onde $\hat{\alpha}$ e $\hat{\beta}$ são as folhagens definidas anteriormente, e temos as folhagens $\hat{\nu}: \hat{S} \rightarrow \hat{Z}$ e $\hat{\mu}: \hat{S} \rightarrow \hat{Y}$ dadas, respectivamente, por $\hat{\mu}(y, z, \sigma):=(y, \sigma)$ e $\hat{\nu}(y, z, \sigma):=(z, \sigma)$. Veja o diagrama:

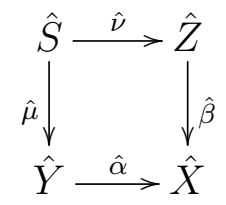

Observação 3.28. O produto fibrado é um diagrama pullback como na Definição 2.21. De fato, note que $\hat{\alpha}((y, \sigma))=\hat{\beta}((z, \sigma))$ para $(y, \sigma) \in \hat{Y},(z, \sigma) \in \hat{Z}$ e $\hat{\alpha} \circ \hat{\mu}=\hat{\beta} \circ \hat{\nu}$.

Agora, vamos definir o produto fibrado $\hat{S}$ como a união de folhas, assim como fizemos para $\hat{Y}$ e $\hat{Z}$. Para $y \in \Gamma / A$ e $z \in \Gamma / B$, seja $\hat{S}_{y, z}$ uma cópia da interseção $\hat{\alpha}\left(\hat{Y}_{y}\right) \cap \hat{\beta}\left(\hat{Z}_{z}\right)=$ $y \mathcal{A} \cap z \mathcal{B}$. Então, como $\hat{Y}$ e $\hat{Z}$ são a união disjuntas de suas folhas, $\hat{S}$ é a união disjunta $\hat{S}=\bigcup_{y, z} \hat{S}_{y, z}$.

Podemos definir em $\hat{S}$ uma $\Gamma$-ação a partir da $\Gamma$-ação definida em $\hat{X}, \hat{Y}$ e $\hat{Z}$, ou seja, para $(y, z, \sigma) \in \hat{S}$ e $g \in \Gamma$, temos

$$
g \cdot(y, z, \sigma):=(g y, g z, g \sigma) .
$$

As propriedades definidas acima para a $\Gamma$-ação valerão para $\hat{S}$ também: 
i) A ação de $\Gamma$ é livre em $\hat{S}$ e induz uma $\Gamma$-ação em $\mathcal{F}(\hat{S})$, permutando as folhas de $\hat{S}$ pela fórmula $g \cdot \hat{S}_{y, z}=\hat{S}_{g y, g z}$.

ii) Os estabilizadores das folhas em $\hat{S}$ serão a interseção dos estabilizadores das folhas de $\hat{Y}$ e de $\hat{Z}$, isto é, $\operatorname{Stab}_{\Gamma}\left(\hat{S}_{y, z}\right)=A^{y^{-1}} \cap B^{z^{-1}}$.

Agora, já estamos em condições de provar o primeiro resultado utilizado na conclusão da demonstração principal.

Lema 3.29. Dados os subgrupos finitamente gerados $A$ e $B$ do grupo livre $\Gamma$ e seus respectivos subgrafos $\mathcal{A}$ e $\mathcal{B}$ definidos anteriormente, valem as igualdades:

i) $\bar{r}(A \backslash \mathcal{A})=\bar{r}(A)$;

ii) $\bar{r}(B \backslash \mathcal{B})=\bar{r}(B)$;

iii) $\bar{r}\left(\left(A^{\bar{y}^{-1}} \cap B^{\bar{z}^{-1}}\right) \backslash(y \mathcal{A} \cap z \mathcal{B})\right)=\bar{r}\left(A^{\bar{y}^{-1}} \cap B^{\bar{z}^{-1}}\right)$.

Demonstração. Para a demonstração dos três itens utilizamos argumentos similares. No caso do item $i$ ), se a árvore $\mathcal{A}$ é não vazia, ela é simplesmente conexa e $A$ é o grupo fundamental do grafo quociente $A \backslash \mathcal{A}$ pelos resultados do Teorema 2.45, então pela definição de posto reduzido na Observação 3.20 , temos $\bar{r}(A \backslash \mathcal{A})=\bar{r}(A)$. Se $\mathcal{A}$ é vazio, então $A$ é trivial pela definição de $\mathcal{A}$, logo vale:

$$
\bar{r}(A \backslash \mathcal{A})=\bar{r}(\emptyset)=0=\bar{r}(1)=\bar{r}(A) .
$$

O caso $i i$ ) segue da mesma maneira. Para o item $i i i)$, se $y \mathcal{A} \cap z \mathcal{B} \neq \emptyset$ então $A^{\bar{y}^{-1}} \cap B^{\bar{z}^{-1}}$ é o grupo fundamental de $\left(A^{\bar{y}^{-1}} \cap B^{\bar{z}^{-1}}\right) \backslash(y \mathcal{A} \cap z \mathcal{B})$ e segue o resultado. Agora, se $y \mathcal{A} \cap z \mathcal{B}=\emptyset$, então $A^{\bar{y}^{-1}} \cap B^{\bar{z}^{-1}}$ é trivial, pois caso contrário existiria um elemento $g$ não trivial nessa interseção e portanto o caminho de $g$ em $\hat{X}$ estaria contido em $y \mathcal{A} \cap z \mathcal{B}$. Então vale a igualdade que queríamos:

$$
\bar{r}\left(\left(A^{\bar{y}^{-1}} \cap B^{\bar{z}^{-1}}\right) \backslash(y \mathcal{A} \cap z \mathcal{B})\right)=\bar{r}(\emptyset)=0=\bar{r}(1)=\bar{r}\left(A^{\bar{y}^{-1}} \cap B^{\bar{z}^{-1}}\right) .
$$

Veremos agora que a partir de imersões, pullbacks e folhagens podemos construir um sistema de grafos. 
Seja $\Gamma$ o grupo livre. Para a construção do $\Gamma$-sistema, comece considerando $\hat{X}$ o grafo de Cayley de $\Gamma$ com a $\Gamma$-ação livre. Sejam $X:=\Gamma \backslash \hat{X}$ o grafo quociente e $p_{X}: \hat{X} \rightarrow X$ a aplicação quociente.

Defina $\alpha: Y \rightarrow X$ e $\beta: Z \rightarrow X$ imersões, dadas como aplicações que podem ser estendidas para recobrimentos de $X$. Seja $S$ o produto fibrado de $Y$ e $Z$ sobre $X$ dado pelo conjunto

$$
S:=\{(y, z) \in Y \times Z \mid \alpha(y)=\beta(z)\}
$$

e sejam $\mu$ e $\nu$ são as projeções para cada coordenada. Veja o diagrama pullback que formamos:

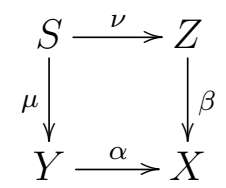

Agora, puxamos para trás o diagrama acima através da projeção $p_{X}: \hat{X} \rightarrow X$, e completamos o resto do sistema, obtendo o $\Gamma$-sistema, que dizemos ser gerado por $\alpha, \beta$ e $p_{X}$.

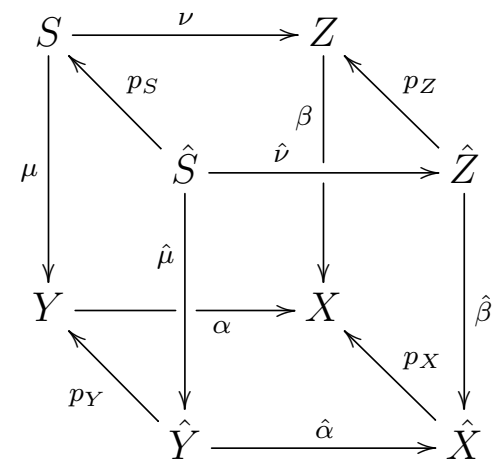

No $\Gamma$-sistema, o quadrado da frente é o diagrama pullback formado pelo produto fibrado $\hat{S}$ de $\hat{Y}$ e $\hat{Z}$ sobre $\hat{X}$, veja na Definição 3.27. As setas diagonais são todas aplicações quocientes, pois $\Gamma$ age livremente em $\hat{X}, \hat{Y}, \hat{Z}$ e $\hat{S}$, obtendo, respectivamente, os grafos quocientes $X:=\Gamma \backslash \hat{X}, Y:=\Gamma \backslash \hat{Y}, Z:=\Gamma \backslash \hat{Z}$ e $S:=\Gamma \backslash \hat{S}$.

Observação 3.30. No sistema acima $p_{X}: \hat{X} \rightarrow X$ é um recobrimento universal, pois $\hat{X}$ é árvore (veja na Seção 2.4). 


\subsubsection{Ordenação e conjuntos ordem-essencial}

Agora iremos ordenar os grafos com os quais estamos trabalhando e, a partir dessa ordenação, obter propriedades e novos conjuntos.

Definição 3.31. Um grafo $\hat{Y}$ é dito ordenado se em $E^{\hat{Y}}$ é dada uma ordem parcial $\leq$ cuja restrição a $E^{K}$ é uma ordem total, para cada componente $K$ de $\hat{Y}$.

Um Г-grafo ordenado é um $\Gamma$-grafo que é ordenado e tal que a ordem parcial em seu conjunto de arestas é preservada pela $\Gamma$-ação.

Vamos definir uma ordem total $\Gamma$-invariante em $E^{\hat{Y}}$. Para isso, assumimos que $\Gamma$ é um grupo ordenado à esquerda, ou seja, existe uma ordem total $<$ em $\Gamma$ tal que,

$$
\text { se } a<b \text {, então } g a<g b \text { para todo } a, b, g \in \Gamma \text {. }
$$

Como $\hat{Y}$ é um grafo com $\Gamma$-ação livre, podemos torná-lo um $\Gamma$-grafo ordenado escolhendo qualquer $\Gamma$-transversal em $E^{\hat{Y}}$, que denotaremos por $\bar{E}^{\hat{Y}}$, e colocando ordem total nele. Então $E^{\hat{Y}}=\Gamma \times \bar{E}^{\hat{Y}}$, e em $E^{\hat{Y}}$ colocamos qualquer ordem lexicográfica.

Observação 3.32. Os grupos livres são ordenados à esquerda, assim o grafo de Cayley de um grupo livre com respeito a qualquer base será uma $\Gamma$-árvore ordenada.

Agora, seja $\hat{Y}$ um grafo localmente finito, ou seja, um grafo no qual cada vértice tem valência finita. Definiremos a propriedade cair em um subconjunto de arestas do grafo induzido $\hat{Y}$ e o que é uma veia de uma aresta. Para isso, relembre que o grafo relativo de $\hat{Y}$ é o grafo $\hat{Y}(E):=V^{\hat{Y}} \cup E$, para um subconjunto $E \subseteq E^{\hat{Y}}$.

Definição 3.33. Dada uma aresta $\sigma \in E^{\hat{Y}}$ e um conjunto de arestas $E \subseteq E^{\hat{Y}} \backslash\{\sigma\}$, dizemos que $\sigma$ cai em $E$ se as componentes do grafo relativo $\hat{Y}(E)$ contendo $\alpha(\sigma)$ e $\omega(\sigma)$ são infinitas ou coincidem.

Vejamos essa definição no desenho de um grafo $X$ qualquer:

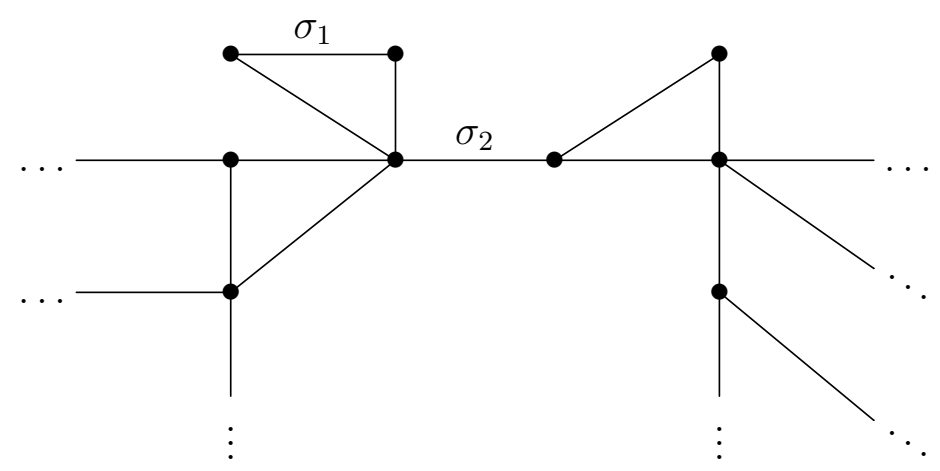


No grafo acima, primeiro considere $E=E^{X} \backslash\left\{\sigma_{1}\right\}$ e vamos ver que a aresta $\sigma_{1}$ cai em $E$. Note que as componentes do grafo relativo que contém $\alpha\left(\sigma_{1}\right)$ e $\omega\left(\sigma_{1}\right)$ coincidem, ou seja, a mesma componente contém o início e o final da aresta $\sigma_{1}$, portanto $\sigma_{1}$ cai em $E$. Se considerarmos agora $E=E^{X} \backslash\left\{\sigma_{2}\right\}$, as componentes do grafo relativo que contém $\alpha\left(\sigma_{2}\right)$ e $\omega\left(\sigma_{2}\right)$ são infinitas e, portanto, $\sigma_{2}$ cai em $E$.

Definição 3.34. Seja $\sigma \in E^{\hat{Y}}$ e considere $E \subseteq E^{\hat{Y}} \backslash\{\sigma\}$. Uma veia de $\sigma$ em E é definida como sendo uma das caracterizações abaixo:

a) Um caminho em $\hat{Y}(E)$ sem auto-interseções de $\alpha(\sigma)$ para $\omega(\sigma)$, é chamado de veia finita;

b) A união de dois raios infinitos em $\hat{Y}(E)$ sem auto-interseções começando em $\alpha(\sigma)$ e $\omega(\sigma)$, respectivamente, é chamada de veia infinita.

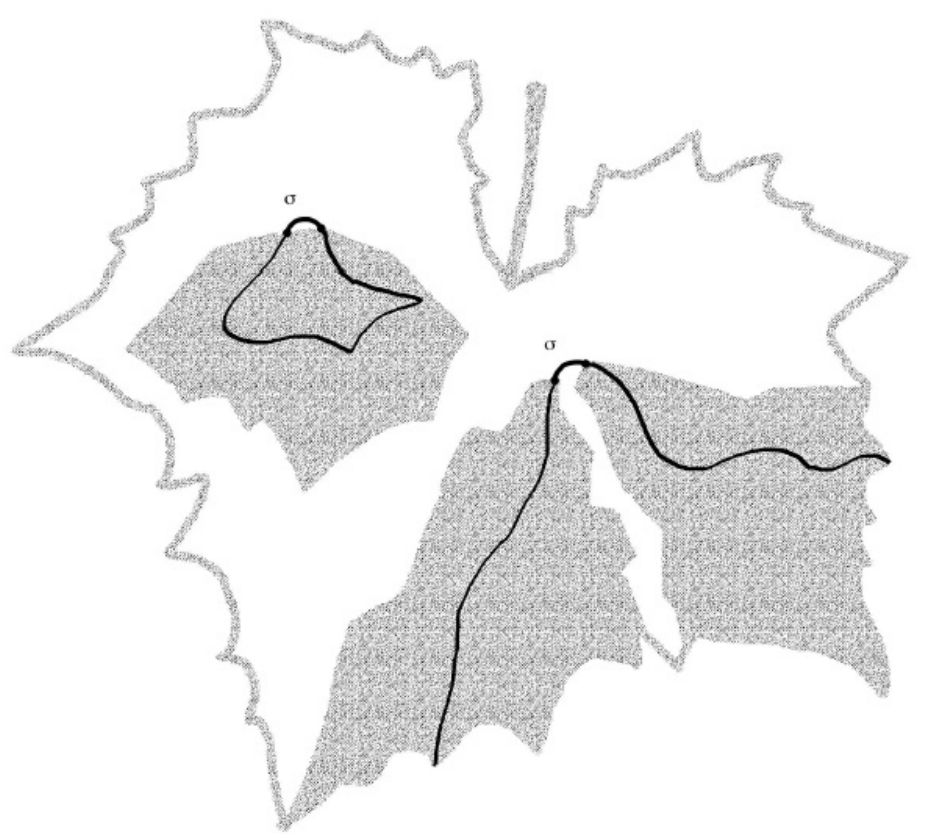

No desenho acima podemos ver a representação de uma veia finita à esquerda e de uma veia infinita à direita. A imagem foi retirada do próprio artigo do Mineyev [18].

Observação 3.35. Note que $\sigma$ cai em $E$ se, e somente se, existe uma veia de $\sigma$ em $E$. De fato, pela definição de $\sigma$ cair em $E$, no caso das componentes do grafo relativo contendo 
$\alpha(\sigma)$ e $\omega(\sigma)$ serem infinitas, temos definida uma veia infinita de $\sigma$ em E e no caso das componentes coincidirem, teremos uma veia finita de $\sigma$ em E.

Além disso, se $\hat{Y}$ é uma floresta então todas as veias são infinitas.

Para um subconjunto $E \subseteq E^{\hat{Y}}$ e uma aresta $\sigma \in E^{\hat{Y}} \backslash E$, iremos denotar um conjunto que ordena as arestas de $E$ em comparação a $\sigma$ como $[E<\sigma]:=\{\tau \in E \mid \tau<\sigma\}$. Com essa definição, definiremos agora os conjuntos de arestas ordem-essencial e ordeminessencial.

Definição 3.36. Uma aresta $\sigma$ em $\hat{Y}$ é dita ser ordem-essencial em $\hat{Y}$ se ela cai em $\left[E^{\hat{Y}}<\sigma\right]$ e ordem-inessencial caso contrário.

Note que dizer que uma aresta $\sigma$ é ordem-essencial em $\hat{Y}$ é equivalente a dizer que existe uma veia de $\sigma$ em $\left[E^{\hat{Y}}<\sigma\right]$ pela Oservação 3.35 .

Denotaremos o conjunto de arestas ordem-essencial e ordem-inessencial de $\hat{Y}$ por $\mathbb{E}^{\hat{Y}}$ e $\mathbb{I}^{\hat{Y}}$, respectivamente. O lema a seguir segue direto das definições dadas até agora.

Lema 3.37. Seja $\Gamma$ um grupo qualquer, $\hat{Y}$ um $\Gamma$-grafo ordenado, $A$ um subgrupo de $\Gamma e$ $\mathcal{A}$ um subgrafo A-invariante de $\hat{Y}$. Então,

a) $\mathcal{A}$ é um A-grafo ordenado com respeito à ordem parcial em $E^{\mathcal{A}}$ restrito de $E^{\hat{Y}}$;

b) $\mathbb{E}^{\mathcal{A}}$ e $\mathbb{I}^{\mathcal{A}}$ são subconjuntos A-invariantes de $\hat{Y}$;

c) $\mathbb{E}^{\mathcal{A}} \subseteq \mathbb{E}^{\hat{Y}}$

Demonstração. a) De fato, por $\mathcal{A}$ ser um subgrafo $A$-invariante do $\Gamma$-grafo ordenado $\hat{Y}$, $\mathcal{A}$ é um $A$-grafo ordenado e a ordem parcial será preservada pela $A$-ação à esquerda no conjunto de arestas $E^{\mathcal{A}}$, pois a ordem é preservada pela $\Gamma$-ação no conjunto todo de $\operatorname{arestas} E^{\hat{Y}}$.

b) Vamos mostrar que $\mathbb{E}^{\mathcal{A}}$ é um subconjunto $A$-invariante de $\hat{Y}$, ou seja, que $A \cdot \mathbb{E}^{\mathcal{A}} \subseteq$ $\mathbb{E}^{\mathcal{A}}$. De fato, se $\sigma \in \mathbb{E}^{\mathcal{A}}$, então $\sigma$ cai em $\left[E^{\mathcal{A}}<\sigma\right]=\left\{\tau \in E^{\mathcal{A}} \mid \tau<\sigma\right\}$. Assim, dado $a \in A$, aplicando a $A$-ação em $\sigma$, a aresta transladada $a \sigma$ cairá no conjunto $\left[E^{\mathcal{A}}<a \sigma\right]=\left\{\tau \in E^{\mathcal{A}} \mid \tau<a \sigma\right\}$, pois a ordem é preservada pela ação, e continuará sendo uma aresta ordem-essencial em $\mathcal{A}$. De maneira análoga, mostra-se que $\mathbb{I}^{\mathcal{A}}$ é subconjunto $A$-invariante de $\hat{Y}$. 
c) Dado $\sigma \in \mathbb{E}^{\mathcal{A}}$, existe uma veia de $\sigma$ em $\left[E^{\mathcal{A}}<\sigma\right]$ pela caracterização dada após a Definição 3.37. Agora, por $\mathcal{A}$ ser subgrafo de $\hat{Y}$, essa veia pertencerá também a $\left[E^{\hat{Y}}<\sigma\right]$. Portanto, $\sigma$ cai em $\left[E^{\hat{Y}}<\sigma\right]$ e, assim, $\mathbb{E}^{\mathcal{A}} \subseteq \mathbb{E}^{\hat{Y}}$.

A proposição a seguir é conhecida como a propriedade deep-fall para grafos.

Proposição 3.38. Suponha que $\hat{Y}$ é um grafo ordenado localmente finito. Se $\sigma \in \mathbb{E}^{\hat{Y}}$, então $\sigma$ cai em $\left[\mathbb{I}^{\hat{Y}}<\sigma\right]$.

Demonstração. Por hipótese, $\hat{Y}$ é um grafo localmente finito, ou seja, a cardinalidade da estrela de cada vértice do grafo é finita, então se substituirmos $\hat{Y}$ por sua componente que contém a aresta $\sigma$, podemos assumir que $\hat{Y}$ é enumerável e que a ordem em $E^{\hat{Y}}$ é total.

Como $\sigma \in \mathbb{E}^{\hat{Y}}, \sigma$ é uma aresta ordem-essencial e por definição cai em $\left[E^{\hat{Y}}<\sigma\right]$. Considere agora o conjunto $\left[\mathbb{E}^{\hat{Y}}<\sigma\right]$, que será o conjunto de arestas pertencentes a $\mathbb{E}^{\hat{Y}}$ menores que $\sigma$ segundo a ordem $<$ estabelecida, e o enumere por $\left\{\sigma_{1}, \sigma_{2}, \ldots\right\}$. Para cada $n \geq 1$, seja o conjunto $\left\{\sigma_{1}, \sigma_{2}, \ldots, \sigma_{n}\right\}$. Já que a ordem em $E^{\hat{Y}}$ é total, podemos renomear o conjunto como $\left\{\tau_{1}, \tau_{2}, \ldots, \tau_{n}\right\}$, de maneira que esse último obedeça à seguinte ordenação $\tau_{1}>\tau_{2}>\cdots>\tau_{n}$. Como $\tau_{1} \in \mathbb{E}^{\hat{Y}}$, existe uma veia de $\tau_{1}$ em $\left[E^{\hat{Y}}<\tau_{1}\right]$ e, do modo como foram considerados, também em $\left[E^{\hat{Y}}<\sigma\right] \backslash\left\{\tau_{1}\right\}$. Considere uma veia qualquer de $\sigma$ em $\left[E^{\hat{Y}}<\sigma\right]$. Se essa veia contém $\tau_{1}$, substitua $\tau_{1}$ pela veia de $\tau_{1}$ em $\left[E^{\hat{Y}}<\tau_{1}\right]$ e remova as auto-interseções, se necessário. Note que o resultado obtido é um subgrafo de $\hat{Y}$ e as componentes desse subgrafo que contém $\alpha(\sigma)$ e $\omega(\sigma)$ formam uma veia de $\sigma \operatorname{em}\left[E^{\hat{Y}}<\sigma\right] \backslash\left\{\tau_{1}\right\}$.

Repetindo esse mesmo argumento indutivamente para as arestas $\tau_{2}, \tau_{3}, \ldots \tau_{n}$, obtemos uma veia de $\sigma$ em $\left[E^{\hat{Y}}<\sigma\right] \backslash\left\{\tau_{1}, \tau_{2}, \ldots, \tau_{n}\right\}=\left[E^{\hat{Y}}<\sigma\right] \backslash\left\{\sigma_{1}, \sigma_{2}, \ldots, \sigma_{n}\right\}$, para cada $n \geq 1$.

Observe agora que, essa sequência de veias consiste de veias finitas com comprimentos uniformemente limitados, ou existe uma subsequência finita ou infinita de veias de $\sigma$ cujos comprimentos convergem para $\infty$. Como $\hat{Y}$ é localmente finito, todas as arestas ordem-essencial, em uma bola grande arbitrária ao redor de $\sigma$, podem ser removidas em alguma etapa finita. Pegando uma subsequência, podemos assumir que a sequência de veias se estabiliza em alguma veia finita de $\sigma$ em $\left[E^{\hat{Y}}<\sigma\right]$, ou seja, ou existe uma veia 
finita limitando, ou o segmento inicial de alguma veia de $\sigma$ em $\left[E^{\hat{Y}}<\sigma\right]$, de comprimento fixado, eventualmente se estabiliza na sequência, e nesse caso temos uma veia infinita limitando. Mas, em ambos os casos, a veia limitante estará em $\left[E^{\hat{Y}}<\sigma\right] \backslash\left\{\sigma_{1}, \ldots, \sigma_{n}\right\}$, para cada $n$, portanto em $\left[\mathbb{I}^{\hat{Y}}<\sigma\right]$. Logo, $\sigma$ cai em $\left[\mathbb{I}^{\hat{Y}}<\sigma\right]$.

Teorema 3.39. Seja $\Gamma$ um grupo qualquer e $\hat{Y}$ um grafo com $\Gamma$-ação livre, cocompacto, isto é $\Gamma \backslash \hat{Y}$ é finito, e ordenado, tal que todas suas arestas são ordem-inessencial. Então $\hat{Y}$ é uma floresta e $\bar{r}(\Gamma \backslash \hat{Y})=0$.

Demonstração. Suponha, por contradição, que $\hat{Y}$ não é uma floresta, então ele possui um circuito simples não trivial. Note que esse circuito está em uma componente de $\hat{Y}$ e a ordem nas arestas da componente é total, portanto existe uma aresta maximal $\tau$ no circuito. Assim, o resto do circuito forma uma veia finita de $\tau$ em $\left[E^{\hat{Y}}<\tau\right]$, então $\tau$ é uma aresta ordem-essencial em $\hat{Y}$, o que contradiz nossa hipótese. Portanto, $\hat{Y}$ é uma floresta.

Mostraremos que $\bar{r}(\Gamma \backslash \hat{Y})=0$. Seja $\hat{Y}^{\prime}$ uma componente de $\hat{Y}$ e seja $\Gamma^{\prime}:=\operatorname{Stab}_{\Gamma}\left(\hat{Y}^{\prime}\right)$, então como $Y:=\Gamma \backslash \hat{Y}$, teremos $Y^{\prime}:=\Gamma^{\prime} \backslash \hat{Y}^{\prime}$ uma componente de $Y$. Agora, como $\hat{Y}$ é um grafo cocompacto, $\Gamma \backslash \hat{Y}$ é finito e a componente $Y^{\prime}=\Gamma^{\prime} \backslash \hat{Y}^{\prime}$ também será finita. Portanto, como $Y^{\prime}$ é uma componente qualquer de $Y$, é suficiente provar que $\bar{r}\left(Y^{\prime}\right)=0$ e então teremos $\bar{r}(Y)=0$.

Note que $\hat{Y}^{\prime}$ é simplesmente conexo e $\Gamma^{\prime}$ age livremente em $\hat{Y}^{\prime}$, então $\Gamma^{\prime}$ é o grupo fundamental de $Y^{\prime}$. Assim, usando o Lema 3.29, temos:

$$
\bar{r}\left(Y^{\prime}\right)=\bar{r}\left(\Gamma^{\prime} \backslash \hat{Y}^{\prime}\right)=\bar{r}\left(\Gamma^{\prime}\right)=\max \left\{0, \operatorname{rank}\left(\Gamma^{\prime}\right)-1\right\}
$$

Vamos supor que $\bar{r}\left(Y^{\prime}\right) \neq 0$ e com essa suposição construir um caminho que possua uma aresta ordem-essencial para $\hat{Y}$, chegando numa contradição com a nossa hipótese. Supondo então que $\bar{r}\left(Y^{\prime}\right) \neq 0, \Gamma^{\prime}$ deverá ter posto pelo menos 2 pelas igualdades acima. Considere então quaisquer dois elementos $g$ e $h$ pertencentes a alguma base em $\Gamma^{\prime}$. Sejam $l_{g}$ e $l_{h}$ seus raios em $\hat{Y}^{\prime}$, isto é, dados dois caminhos finitos $p$ e $q$ em $\hat{Y}^{\prime}, l_{g}$ e $l_{h}$ serão concatenações periódicas biinfinitas sem cancelamentos da seguinte forma, respectivamente:

$$
\begin{aligned}
& \ldots \cdot g^{-2} p \cdot g^{-1} p \cdot p \cdot g p \cdot g^{2} p \cdot \ldots \\
& \ldots \cdot h^{-2} q \cdot h^{-1} q \cdot q \cdot h q \cdot h^{2} q \cdot \ldots
\end{aligned}
$$


Seja $E^{p}$ o conjunto de arestas em $p$. Como $p$ está em uma componente de $\hat{Y}$, a restrição da ordem em $E^{\hat{Y}}$ para $E^{p}$ é total, e então existe uma aresta maximal $\xi$ em $E^{p}$. Note que teremos $g \xi \neq \xi$, pois $g$ é elemento da base e a ação é livre. Então, podemos assumir que $\xi>g \xi$ ou $\xi<g \xi$. Assumindo que $\xi>g \xi$, como a ordem é invariante a esquerda, teremos

$$
\ldots>g^{-2} \xi>g^{-1} \xi>\xi>g \xi>g^{2} \xi>\ldots
$$

Por $\xi$ ser aresta maximal em $E^{p}$, para cada $\tau \in E^{p} \backslash\{\xi\}$, temos $\xi>\tau$ e então $g^{n} \xi>g^{n} \tau$, para todo $n \in \mathbb{Z}$. Portanto, para cada $n \in \mathbb{Z}$, a aresta $g^{n} \xi$ é a maior aresta no raio $g^{n} p \cdot g^{n+1} p \cdot g^{n+2} p \cdot \ldots$. Se removermos algumas arestas iniciais e renomearmos ciclicamente o caminho $p$, podemos assumir que $g^{n} \xi$ é a aresta inicial nesse raio. Agora, se assumirmos que $\xi<g \xi$ então teremos

$$
\ldots<g^{-2} \xi<g^{-1} \xi<\xi<g \xi<g^{2} \xi<\ldots
$$

e, como antes, $g^{n} \xi>g^{n} \tau$ para $\tau \in E^{p} \backslash\{\xi\}$. Assim, $g^{n} \xi$ será a maior aresta no raio $\ldots \cdot g^{n-2} p \cdot g^{n-1} p \cdot g^{n} p$, e podemos assumir que $g^{n} \xi$ é a aresta terminal.

O mesmo pode ser feito para $h$ e $l_{h}$ considerando $\eta$ a aresta maximal em $q$ e escrevendo inequações similares. Temos então quatro casos a considerar: $\xi>g \xi$ e $\eta>h \eta, \xi>g \xi$ e $\eta<h \eta, \xi<g \xi$ e $\eta>h \eta$, e $\xi<g \xi$ e $\eta<h \eta$. Vamos assumir o primeiro, $\xi>g \xi$ e $\eta>h \eta$, os demais casos serão análogos. Sendo assim, teremos dois raios

$$
g^{n} p \cdot g^{n+1} p \cdot g^{n+2} p \cdot \ldots \quad \text { e } \quad h^{k} q \cdot h^{k+1} q \cdot h^{k+2} q \cdot \ldots
$$

onde suas respectivas arestas iniciais $g^{n} \xi$ e $h^{k} \eta$ são as maiores arestas desses dois raios.

Como $g$ e $h$ são elementos distintos da base, esse raios eventualmente tornam-se disjuntos quando $n$ e $k$ crescem. Podemos conectar os vértices iniciais desses dois raios por um caminho finito $r$, removemos autointerseções e pegamos $n$ e $k$ suficientemente grandes para obter um caminho biinfinito

$$
\ldots \cdot g^{n+2} p^{-1} \cdot g^{n+1} p^{-1} \cdot g^{n} p^{-1} \cdot r \cdot h^{k} q \cdot h^{k+1} q \cdot h^{k+2} q \cdot \ldots
$$

Veja o desenho dos dois raios ligados pelo caminho finito $r$ :

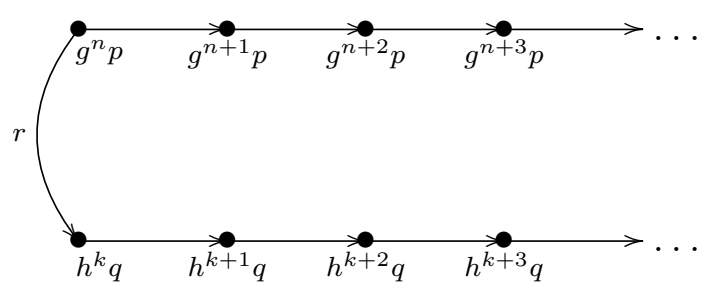


Agora, considere $\omega$ a aresta maximal dentre as arestas em $r$ e as $\operatorname{aretas} g^{n} \xi$ e $h^{k} \eta$. Note que cada aresta diferente de $\omega$ no caminho biinfinito definido acima é estritamente menor que $\omega$. Então $\omega$ é aresta ordem-essencial para $\hat{Y}^{\prime}$. Como $\hat{Y}^{\prime}$ é uma componente de $\hat{Y}, \omega$ é aresta ordem-essencial para $\hat{Y}$, o que é uma contradição com a hipótese de que todas as suas arestas são ordem-inessencial. Portanto, $\bar{r}\left(Y^{\prime}\right)=0$ e, assim, $\bar{r}(Y)=\bar{r}(\Gamma \backslash \hat{Y})=0$.

Observação 3.40. Mostramos no teorema acima que $\bar{r}(Y)=0$, e por $Y=\Gamma \backslash \hat{Y}$ ser finito, essa condição implica que $Y$ é um jardim finito, ou seja, uma união disjunta de árvores e flores, pois são esses elementos que possuem posto reduzido igual a zero.

Veremos agora que se considerarmos $\hat{Y}$ sob algumas condições, então o posto reduzido do grafo quociente $Y$ será igual à cardinalidade do conjunto de arestas ordem-essencial maximal de $Y$, e esse será o principal resultado utilizado na demonstração da conjectura.

Teorema 3.41. Sejam $\Gamma$ um grupo qualquer e $\hat{Y}$ um grafo com $\Gamma$-ação livre, cocompacto e ordenado e considere $Y:=\Gamma \backslash \hat{Y}, \mathbb{E}^{Y}:=\Gamma \backslash \mathbb{E}^{\hat{Y}}, \mathbb{I}^{Y}:=\Gamma \backslash \mathbb{I}^{\hat{Y}}$. Então $\mathbb{E}^{Y}$ é o conjunto essencial maximal em $Y$ e, em particular, $\bar{r}(Y)=\# \mathbb{E}^{Y}$.

Demonstração. Inicialmente, considere $Y\left(\mathbb{I}^{Y}\right):=Y \backslash \mathbb{E}^{Y}$, ou seja, o grafo quociente $Y$ apenas com o conjunto de arestas ordem-inessencial, sem o conjunto de arestas ordemessencial. Seja $\sigma \in \mathbb{E}^{\hat{Y}}$, então pela Proposição 3.38, $\sigma$ tem uma veia em $\left[\mathbb{I}^{\hat{Y}}<\sigma\right]$. Por $\hat{Y}$ ser uma floresta, essa veia será infinita. Considere agora a aplicação quociente $q: \hat{Y} \rightarrow Y$. Note que cada raio da veia infinita em $\left[\mathbb{I}^{\hat{Y}}<\sigma\right]$ será levado não injetivamente pela aplicação $q$ no quociente finito $Y\left(\mathbb{I}^{Y}\right)$. Como a $\Gamma$-ação em $\hat{Y}$ é livre, a aplicação quociente $q$ é um recobrimento (veja Exemplo 2.44). Assim, a estrela de cada vértice $v \in V^{\hat{Y}}$ é levada bijetivamente na estrela do vértice $q(v)$ em $Y$, então a imagem de cada raio da veia infinita pela aplicação quociente precisa ter pelo menos um laço em $Y\left(\mathbb{I}^{Y}\right)$. Então, a componente de $Y\left(\mathbb{I}^{Y}\right)$ contendo $q(\alpha(\sigma))$ não é uma árvore, assim como a componente contendo $q(\omega(\sigma))$. Logo, pela caracterização dada para conjuntos essenciais após a Definição $3.23, \mathbb{E}^{Y}$ é essencial em $Y$.

Agora, note que as arestas em $\mathbb{I}^{\hat{Y}}$ são ordem-inessencial em $\hat{Y}$, então elas permanecem ordem-inessencial no grafo menor $\hat{Y}\left(\mathbb{I}^{\hat{Y}}\right)=\hat{Y} \backslash \mathbb{E}^{\hat{Y}}$. Pelo Teorema 3.39, como todas as arestas em $\hat{Y}\left(\mathbb{I}^{\hat{Y}}\right)$ são ordem-inessencial, então $\bar{r}\left(Y\left(\mathbb{I}^{Y}\right)\right)=0$. Portanto, por $\mathbb{E}^{Y}$ ser um 
conjunto essencial em $Y$ temos $\bar{r}\left(Y \backslash \mathbb{E}^{Y}\right)=\bar{r}(Y)-\# \mathbb{E}^{Y}$ e, assim, $\mathbb{E}^{Y}$ é um conjunto essencial maximal em $Y$, pois

$$
\bar{r}\left(Y\left(\mathbb{I}^{Y}\right)\right)=\bar{r}\left(Y \backslash \mathbb{E}^{Y}\right)=\bar{r}(Y)-\# \mathbb{E}^{Y}=0
$$

e, então, $\bar{r}(Y)=\# \mathbb{E}^{Y}$.

Com esses resultados, podemos provar agora que a conjectura é válida. Para isso, sendo $A \backslash \Gamma / B$ o conjunto de classes laterais duplas $A g B$, onde $g \in \Gamma$, vamos considerar de maneira análoga à seção anterior, $s: A \backslash \Gamma / B \rightarrow \Gamma$ uma seção da aplicação quociente $\Gamma \rightarrow A \backslash \Gamma / B$. Além disso, seja $x \in s(A \backslash \Gamma / B)$ tal que $A^{x} \cap B$ é não trivial.

Teorema 3.42 (SHNC). Seja $\Gamma$ um grupo livre e sejam $A$ e $B$ subgrupos finitamente gerados de $\Gamma$. Então

$$
\sum_{x \in s(A \backslash \Gamma / B)} \bar{r}\left(A^{x} \cap B\right) \leq \bar{r}(A) \bar{r}(B) .
$$

Demonstração. Inicialmente, considere o sistema de grafos construído anteriormente (3.5), o qual chamamos de $\Gamma$-sistema gerado pelas imersões $\alpha$ e $\beta$ e pela projeção $p_{X}$, onde o quadrado da frente é o diagrama pullback formado pelo produto fibrado $\hat{S}$ de $\hat{Y}$ e $\hat{Z}$ sobre $\hat{X}$, o quadrado de trás é o diagrama pullback formado pelo produto fibrado $S$ de $Y$ e $Z$ sobre $X$ e as setas diagonais são aplicações quocientes:

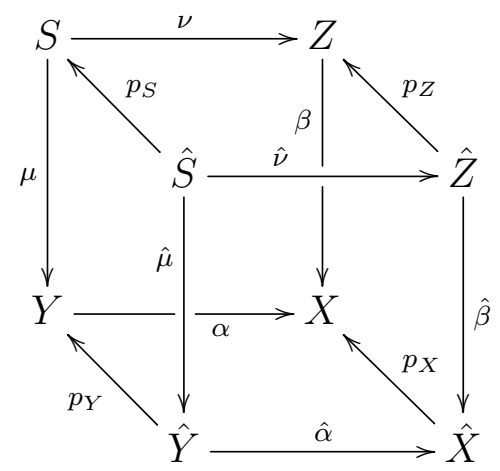

Podemos colocar uma ordem $\Gamma$-invariante no conjunto $E^{\hat{X}}$ de arestas do grafo $\hat{X}$, como a definida anteriormente no começo da seção. Essa ordem pode ser puxada para trás de $\hat{X}$ para cada folha de $\hat{Y}, \hat{Z}, \hat{S}$ pelas folhagens $\hat{\alpha}, \hat{\beta}, \hat{\alpha} \circ \hat{\mu}$.

Como $\hat{S}$ é o produto fibrado de $\hat{Y}$ e $\hat{Z}$ sobre $\hat{X}$, podemos considerar a aplicação inclusão nas arestas $\hat{\varphi}: E^{\hat{S}} \rightarrow E^{\hat{Y}} \times E^{\hat{Z}}$, onde para $\tau=(y, z, \sigma) \in \hat{S}$ temos:

$$
\hat{\varphi}(\tau)=(\hat{\mu}(\tau), \hat{\nu}(\tau))=((y, \sigma),(z, \sigma)) .
$$


Como as folhagens $\hat{\mu}$ e $\hat{\nu}$ comutam com a $\Gamma$-ação pelas propriedades da $\Gamma$-ação após a Definição 3.25, dado $g \in \Gamma$ temos $g \cdot \hat{\mu}(\tau)=\hat{\mu}(g \tau)$ e $g \cdot \hat{\nu}(\tau)=\hat{\nu}(g \tau)$. Então, a aplicação $\hat{\varphi}$ induz uma aplicação $\varphi: E^{S} \rightarrow E^{Y} \times E^{Z}$ nos respectivos conjuntos quocientes. Vamos provar que $\varphi$ é injetiva.

Sejam $\tau=(y, z, \sigma)$ e $\tau^{\prime}=\left(y^{\prime}, z^{\prime}, \sigma^{\prime}\right) \in S$ e suponha que, para $g, h \in \Gamma, \mu\left(y^{\prime}, z^{\prime}, \sigma^{\prime}\right)=$ $\mu(g \cdot(y, z, \sigma))$ e $\nu\left(y^{\prime}, z^{\prime}, \sigma^{\prime}\right)=\nu(h \cdot(y, z, \sigma))$, ou seja, $\left(y^{\prime}, \sigma^{\prime}\right)=(g y, g \sigma)$ e $\left(z^{\prime}, \sigma^{\prime}\right)=(h z, h \sigma)$. Então,

$$
\sigma^{\prime}=g \sigma=h \sigma \Rightarrow g \sigma=h \sigma \Rightarrow g=h,
$$

pois a $\Gamma$-ação em $\hat{X}$ é livre. Portanto, $\left(y^{\prime}, z^{\prime}, \sigma^{\prime}\right)=(g y, g z, g \sigma)$, assim, $(y, z, \sigma)$ e $\left(y^{\prime}, z^{\prime}, \sigma^{\prime}\right)$ estão na mesma órbita de $\Gamma$. Em outras palavras, provamos que $\varphi\left(\tau^{\prime}\right)=\varphi(\Gamma \cdot \tau) \Rightarrow \tau^{\prime}=$ $\Gamma \cdot \tau$, portanto $\varphi$ é injetiva.

Vamos considerar agora o conjunto de arestas ordem-essencial dos conjuntos de arestas $E^{\hat{S}}, E^{\hat{Y}}, E^{\hat{Z}}$. Pelo item $c$ ) do Lema 3.37, folhagens levam arestas ordem-essencial em arestas ordem-essencial, assim $\hat{\mu}\left(\mathbb{E}^{\hat{S}}\right) \subseteq \mathbb{E}^{\hat{Y}}$ e $\hat{\nu}\left(\mathbb{E}^{\hat{S}}\right) \subseteq \mathbb{E}^{\hat{Z}}$. Podemos considerar também o quociente desses conjuntos por $\Gamma$, denotando $\mathbb{E}^{Y}:=\Gamma \backslash \mathbb{E}^{\hat{Y}} \subseteq E^{Y}, \mathbb{E}^{Z}:=\Gamma \backslash \mathbb{E}^{\hat{Z}} \subseteq E^{Z} \mathrm{e}$ $\mathbb{E}^{S}:=\Gamma \backslash \mathbb{E}^{\hat{S}} \subseteq E^{S}$. Obtemos assim, o seguinte diagrama:

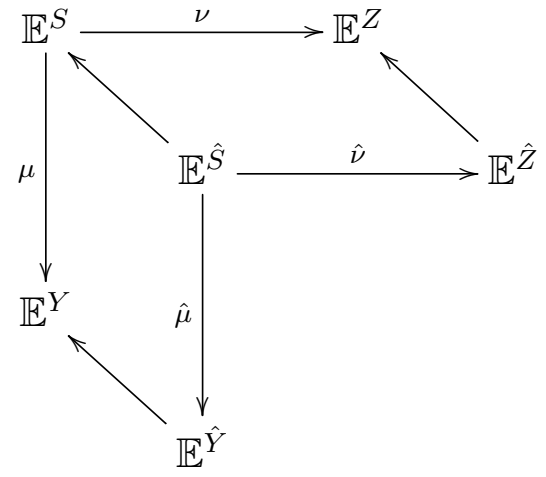

Agora, note que podemos restringir a aplicação $\hat{\varphi}$, definida acima, para $\mathbb{E}^{\hat{S}}$ tomando valores em $\mathbb{E}^{\hat{Y}} \times \mathbb{E}^{\hat{Z}}$, ou seja, obtendo uma aplicação $\hat{\varphi}: \mathbb{E}^{\hat{S}} \rightarrow \mathbb{E}^{\hat{Y}} \times \mathbb{E}^{\hat{Z}}$. Da mesma forma, essa aplicação induzirá uma aplicação injetiva $\varphi: \mathbb{E}^{S} \rightarrow \mathbb{E}^{Y} \times \mathbb{E}^{Z}$ nos quocientes. Então temos $\# \mathbb{E}^{S} \leq \# \mathbb{E}^{Y} \# \mathbb{E}^{Z}$.

Pelo Teorema 3.41, segue que $\bar{r}(S) \leq \bar{r}(Y) \bar{r}(Z)$. Note que, pela Observação 3.26, $Y:=\Gamma \backslash \hat{Y} \cong A \backslash \mathcal{A}$ e $Z:=\Gamma \backslash \hat{Z} \cong B \backslash \mathcal{B}$. Portanto, por essa observação e pelo Lema 3.29 
temos:

$$
\bar{r}(S) \leq \bar{r}(A \backslash \mathcal{A}) \bar{r}(B \backslash \mathcal{B})=\bar{r}(A) \bar{r}(B) .
$$

Resta mostrar então que $\bar{r}(S)=\sum_{x \in s(A \backslash \Gamma / B)} \bar{r}\left(A^{x} \cap B\right)$.

Para isso, considere a $\Gamma$-ação diagonal à esquerda em $(\Gamma / A) \times(\Gamma / B)$ e a aplicação

$$
\begin{aligned}
\theta: s(A \backslash \Gamma / B) & \longrightarrow(\Gamma / A) \times(\Gamma / B) \\
x & \longmapsto\left(x^{-1} A, 1 B\right)
\end{aligned}
$$

Note que $\theta$ é injetiva, pois dados $x, x^{\prime} \in s(A \backslash \Gamma / B)$ distintos, temos

$$
\theta(x)=\left(x^{-1} A, 1 B\right) \neq\left(x^{-1} A, 1 B\right)=\theta\left(x^{\prime}\right) .
$$

Além disso, podemos mostrar que a imagem de $\theta$ intersecta cada $\Gamma$-órbita em $(\Gamma / A) \times$ $(\Gamma / B)$ exatamente uma vez, ou seja, dado um elemento $(\bar{y} A, \bar{z} B) \in(\Gamma / A) \times(\Gamma / B)$, onde $\bar{y}, \bar{z} \in \Gamma$, queremos ver que

$$
\theta(s(A \backslash \Gamma / B)) \cap(\Gamma \cdot(\bar{y} A, \bar{z} B))=\left\{\left(x^{-1} A, 1 B\right)\right\} .
$$

De fato, existe um elemento $x \in s(A \backslash \Gamma / B)$ tal que $\bar{y}^{-1} \bar{z} \in A x B$. Então, se $a \in A$ e $b \in B$ temos $\bar{y}^{-1} \bar{z}=a x b \Rightarrow \bar{z} b^{-1}=\bar{y} a x$. Assim,

$$
\bar{z} b^{-1} \cdot\left(x^{-1} A, 1 B\right)=\left(\bar{z} b^{-1} x^{-1} A, \bar{z} b^{-1} B\right)=\left(\bar{y} a x x^{-1} A, \bar{z} B\right)=(\bar{y} A, \bar{z} B) .
$$

Portanto, $\Gamma \cdot(\bar{y} A, \bar{z} B)$ contém $\left(x^{-1} A, 1 B\right)=\theta(x)$, onde $x \in s(A \backslash \Gamma / B)$. Para provar a unicidade, considere $x, x^{\prime} \in s(A \backslash \Gamma / B)$ tais que para $g \in \Gamma$, temos

$$
g \cdot\left(x^{-1} A, 1 B\right)=\left(x^{-1} A, 1 B\right) \Rightarrow\left(g x^{-1} A, g B\right)=\left(x^{\prime-1} A, 1 B\right) .
$$

Assim, $g \in B$ e

$$
g x^{-1} A=x^{-1} A \Rightarrow x^{\prime} g x^{-1} A=A \Rightarrow x^{\prime} g x^{-1} \in A \Rightarrow x^{\prime} g x^{-1}=a \Rightarrow x=a^{-1} x^{\prime} g .
$$

Logo, $x=a^{-1} x^{\prime} g \in A x^{\prime} B$ e como $x \in A x B$, segue que $x=x^{\prime}$.

Isso mostra que o conjunto $\left\{\hat{S}_{x^{-1} A, 1 B} \mid x \in s(A \backslash \Gamma / B)\right\}$ tem exatamente uma folha em cada $\Gamma$-órbita do conjunto das folhas $\mathcal{F}(\hat{S})$ de $\hat{S}$. Além disso, vamos calcular o estabilizador da folha $\hat{S}_{x^{-1} A, 1 B}$ :

$$
\begin{aligned}
\gamma \in \operatorname{Stab}_{\Gamma}\left(\hat{S}_{x^{-1} A, 1 B}\right) & \Leftrightarrow\left(\gamma x^{-1} A, \gamma B\right)=\left(x^{-1} A, 1 B\right) \Leftrightarrow \gamma x^{-1} A=x^{-1} A, \gamma B=B \\
& \Leftrightarrow \gamma \in x^{-1} A x, \gamma \in B .
\end{aligned}
$$


Então, o estabilizador de $\hat{S}_{x^{-1} A, 1 B}$ é $A^{x} \cap B$ e, como visto anteriormente, $\hat{S}=\bigcup_{y, z} \hat{S}_{y, z}$, assim, temos

$$
S=\Gamma \backslash \hat{S}=\Gamma \backslash\left(\bigcup_{(y, z) \in(\Gamma / A) \times(\Gamma / B)} \hat{S}_{y, z}\right)=\bigcup_{x \in s(A \backslash \Gamma / B)}\left(A^{x} \cap B\right) \backslash \hat{S}_{x^{-1} A, 1 B} .
$$

Pelo Lema 3.29, segue que

$$
\bar{r}(S)=\sum_{x \in s(A \backslash \Gamma / B)} \bar{r}\left(\left(A^{x} \cap B\right) \backslash \hat{S}_{x^{-1} A, 1 B}\right)=\sum_{x \in s(A \backslash \Gamma / B)} \bar{r}\left(A^{x} \cap B\right) .
$$

Portanto, $\sum_{x \in s(A \backslash \Gamma / B)} \bar{r}\left(A^{x} \cap B\right) \leq \bar{r}(A) \bar{r}(B)$.

Assim, está demonstrada a versão forte da conjectura de Hanna Neumann e, portanto, a Conjectura de Hanna Neumann é verdadeira.

\subsection{A demonstração de Warren Dicks}

Em 2011, após estudar as demonstrações da conjectura dadas independentemente por Joel Friedman em [10] e Igor Mineyev em [17], Warren Dicks apresentou simplificações para as duas versões. A que nos interessa é a simplificação dada para a versão de Mineyev (veja em [8]), a qual utiliza teoria de grafos. A demonstração que faremos será baseada também em [9], que é um e-mail que Warren Dicks enviou ao próprio Igor Mineyev.

Para a construção da demonstração de Dicks, vamos considerar $\Gamma=\Gamma(a, b)$ grupo livre de posto dois e $G, A$ e $B$ subgrupos de $\Gamma$. Nosso objetivo é provar o teorema abaixo:

Teorema 3.43 (SHNC). Seja $\Gamma$ grupo livre e sejam A e B subgrupos finitamente gerados de $\Gamma$. Seja $S$ um subconjunto de $\Gamma$ tal que a aplicação $S \rightarrow A \backslash \Gamma / B$, dada por $s \mapsto A s B$, é bijetiva. Então

$$
\sum_{x \in S} \bar{r}\left(A^{x} \cap B\right) \leq \bar{r}(A) \bar{r}(B)
$$

Seja $T$ o grafo de Cayley de $\Gamma$, então $T$ é uma árvore e $\Gamma$ age livremente em $T$. Para cada subgrupo $G$ de $\Gamma$, seja $T_{G}$ a menor $G$-subárvore de $T$, ou seja, $T_{G}$ é $G$-invariante: $\forall g \in G, g \cdot T_{G} \subseteq T_{G}$. Considere então o grafo quociente $G \backslash T_{G}$, cujo grupo fundamental será o próprio grupo $G$. Note que essas definições são similares com as definidas na 
subseção anterior 3.3.1, de construção de folhagens e sistemas. Então, pelo Lema 3.29 segue que $\bar{r}\left(G \backslash T_{G}\right)=\bar{r}(G)$.

Agora, se $A \leq G$, temos a inclusão natural $T_{A} \longrightarrow T_{G}$, a qual induz uma aplicação $A \backslash T_{A} \longrightarrow G \backslash T_{G}$. Então, se considerarmos a interseção $A \cap B$, temos $A \cap B \leq A$ e $A \cap B \leq B$ e, assim, $T_{A \cap B} \subseteq T_{A}$ e $T_{A \cap B} \subseteq T_{B}$. Logo, existe a aplicação injetiva:

$$
(A \cap B) \backslash T_{A \cap B} \longrightarrow A \backslash T_{A} \times B \backslash T_{B} .
$$

Observação 3.44. O grafo quociente $(A \cap B) \backslash T_{A \cap B}$ pode ser visto como o descrito no Exemplo 2.15, o qual é um subgrafo do grafo produto direto $A \backslash T_{A} \times B \backslash T_{B}$.

Note que, como $A$ e $B$ são subgrupos finitamente gerados, a interseção $A \cap B$ também é. Além disso, cada componente conexa na imagem corresponde, por conjugação, a um subgrupo $x^{-1} A x \cap y^{-1} B y$. Assim, temos a aplicação natural injetiva:

$$
\bigcup_{x \in S}\left(A^{x} \cap B\right) \backslash T_{A^{x} \cap B} \longrightarrow A \backslash T_{A} \times B \backslash T_{B} .
$$

Note que o grupo fundamental do grafo $\left(A^{x} \cap B\right) \backslash T_{A^{x} \cap B}$, como observado, será o grupo $A^{x} \cap B$. Então,

$$
\sum_{x \in S} \bar{r}\left(A^{x} \cap B\right)=\sum_{x \in S} \bar{r}\left(\left(A^{x} \cap B\right) \backslash T_{A^{x} \cap B}\right)
$$

será limitado por causa da aplicação injetiva definida acima.

Agora, suponha que para todo $G \leq \Gamma$ exista uma $G$-subárvore $\mathcal{T}_{G}$, em particular para os subgrupos $A, B$ e $A^{x} \cap B$, tal que existe a aplicação injetiva

$$
\bigcup_{x \in S}\left(A^{x} \cap B\right) \backslash \mathcal{T}_{A^{x} \cap B} \longrightarrow A \backslash \mathcal{T}_{A} \times B \backslash \mathcal{T}_{B},
$$

e que, para cada, $G \leq \Gamma$ satisfaça

$$
\bar{r}(G)=\left|G \backslash E^{\mathcal{T}_{G}}\right| .
$$

Se restringíssemos a aplicação acima para o conjunto de arestas de cada subárvore, teríamos pela injetividade da aplicação

$$
\sum_{x \in S}\left|\left(A^{x} \cap B\right) \backslash E^{\mathcal{T}_{A^{x}} \cap B}\right| \leq\left|A \backslash E^{\mathcal{T}_{A}}\right|\left|B \backslash E^{\mathcal{T}_{B}}\right| .
$$


E, assim, pela igualdade (3.6) estaria provada a conjectura:

$$
\sum_{x \in S} \bar{r}\left(A^{x} \cap B\right) \leq \bar{r}(A) \bar{r}(B)
$$

Mostraremos que as subárvores satisfazendo as condições acima existem, provando, assim, o teorema desejado.

Para essa construção, considere $T$ o grafo de Cayley do grupo livre $\Gamma$ gerado por $S=\{a, b\}$, então, pela Definição 2.29 do grafo de Cayley, $V^{T}=\Gamma$ e $E^{T}=\Gamma \times S$. Vamos ordenar o conjunto de arestas $E^{T}$ de $T$ lexicograficamente, colocando ordem invariante à esquerda em $\Gamma$ e ordem total no conjunto $S=\{a, b\}$. Então a ordem lexicográfica em $E^{T}$ é $\Gamma$-invariante à esquerda e vamos denotá-la por $\prec$.

Definida essa ordenação, podemos definir dois conjuntos fundamentais para o que queremos provar, o conjunto de pontes e ilhas da subárvore $T_{G}$.

Definição 3.45. Uma aresta $e \in E^{T_{G}}$ é uma ponte se existe algum caminho biinfinto em $T_{G}$, isto é, uma reta infinita nas duas direções tal que cada vértice tem valência 2 , no qual e é a maior aresta em relação à ordem $\prec$. Denotaremos por $B(G)$ o conjunto de pontes de $T_{G}$.

Definição 3.46. Cada componente conexa de $T_{G} \backslash B(G)$ é chamada uma ilha de $T_{G}$. Denotaremos o conjunto de ilhas de $T_{G}$ por $I(G)$.

Observação 3.47. 1. Considerando a ordem estabelecida, o conjunto $B(G)$ é invariante pela ação de $G$, já que a ordem é invariante à esquerda, e essa ação é livre, pois para $e \in B(G)$ temos $g \cdot e \neq$ e para todo $1 \neq g \in G$. Assim como, o conjunto de ilhas $I(G)$ será $G$-invariante, pelo mesmo argumento utilizado para o conjunto $B(G)$.

2. Note que as definições dos conjuntos de pontes e ilhas assemelham-se, respectivamente, com os conjuntos de arestas ordem-essencial e ordem-inessencial vistos na Definição $3.3 \%$.

Agora, para cada subgrupo $G$ de $\Gamma$ vamos definir a subárvore que desejamos para provar o resultado principal. 
Definição 3.48. Para cada $G \leq \Gamma$, seja $\mathcal{T}_{G}$ a $G$-subárvore com conjunto de arestas $E^{\mathcal{T}_{G}}=B(G)$ e conjunto de vértices $V^{\mathcal{T}_{G}}=I(G)$.

Note que o grafo $\mathcal{T}_{G}$ é um subgrafo do grafo $T_{G}$. Além disso, se $A \leq G$ temos $T_{A} \subseteq T_{G}$, então se $e$ é uma ponte de $T_{A}$, e será uma ponte de $T_{G}$. Isso induz uma aplicação injetiva $\mathcal{T}_{A} \longrightarrow \mathcal{T}_{G}$ e, assim, temos a aplicação $A \backslash \mathcal{T}_{A} \longrightarrow G \backslash \mathcal{T}_{G}$.

Então, voltando a considerar os subgrupos $A, B$ e $A^{x} \cap B$ e as respectivas subárvores $T_{A}, T_{B}$ e $T_{A^{x} \cap B}$, a aplicação injetiva $\bigcup_{x \in S}\left(A^{x} \cap B\right) \backslash T_{A^{x} \cap B} \longrightarrow A \backslash T_{A} \times B \backslash T_{B}$ induz a aplicação injetiva nas novas subárvores que acabamos de definir:

$$
\bigcup_{x \in S}\left(A^{x} \cap B\right) \backslash \mathcal{T}_{A^{x} \cap B} \longrightarrow A \backslash \mathcal{T}_{A} \times B \backslash \mathcal{T}_{B}
$$

Portanto, como observado anteriormente, para provarmos a conjectura (3.7) resta mostrar que para cada subgrupo $G \leq \Gamma$ temos

$$
\bar{r}(G)=\left|G \backslash E^{\mathcal{T}_{G}}\right|=|G \backslash B(G)|
$$

Para isso, vamos analisar os possíveis valores do posto do grupo $G$ e provar alguns resultados necessários para chegarmos a essa conclusão.

Note que se $\operatorname{rank}(G)=0$, o grupo $G$ é trivial, então tanto $T_{G}$ como $B(G)$ são conjuntos vazios, assim $\mathcal{T}_{G}$ é vazio e $\bar{r}(G)=0=|G \backslash B(G)|$. Logo o resultado é válido. Quando $\operatorname{rank}(G)=1$, temos o seguinte teorema:

Teorema 3.49. Se $G=\langle g\rangle$, onde $g \in \Gamma \backslash\{1\}$, não existem pontes em $T_{G}$.

Demonstração. Seja $g \in \Gamma \backslash\{1\}$, se $G=\langle g\rangle$ então $T_{G}=T_{\langle g\rangle}$ é justamente o caminho biinfinito de $g$, ou seja, a reta infinita em ambas as direções com vértices de valência 2 , e será o único da subárvore.

Considere em $T_{G}$ um vértice $v$ qualquer e $p$ o caminho ligando $v$ e $g v$. Seja $e$ a maior aresta em $p$ em relação à ordem $\prec$ estabelecida. Mas note que se $g e \prec e$ então $e \prec g^{-1} e$, pois a ordem é invariante à esquerda, e teremos $\cdots \prec g p \prec p \prec g^{-1} p \prec \ldots$ Logo não exite maior aresta nesse eixo, ou seja, não existe ponte em $T_{G}$.

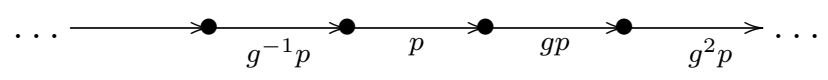


No caso do teorema acima, $B(G)$ é um conjunto vazio e teremos que $\mathcal{T}_{G}$ é uma ilha. Agora, quando $\operatorname{rank}(G) \geq 2$ podemos provar que $B(G)$ será não vazio:

Teorema 3.50. Se $\operatorname{rank}(G) \geq 2$ então $B(G)$ é um conjunto não vazio.

Demonstração. Vamos supor que $G$ é um grupo livre de posto dois. Suponha que $g, h \in G$ geram $G$ e consigere os caminhos biinfinitos de $g$ e $h$, ou seja, $T_{\langle g\rangle}$ e $T_{\langle h\rangle}$, como considerado no Teorema 3.49 anterior. Sejam $v$ um vértice qualquer em $T_{\langle g\rangle}$ e $p$ o caminho reduzido ligando $v$ a $g v, w$ um vértice em $T_{\langle h\rangle}$ e $r$ o caminho reduzido ligando $w$ a $h w$. Considere também $q$ um caminho reduzido de menor comprimento possível ligando $T_{\langle g\rangle}$ e $T_{\langle h\rangle}$ nos vértices $v$ e $w$. Podemos substituir $g$ por $g^{-1}$ no eixo $T_{\langle g\rangle}$ se preciso, e assim obtemos:

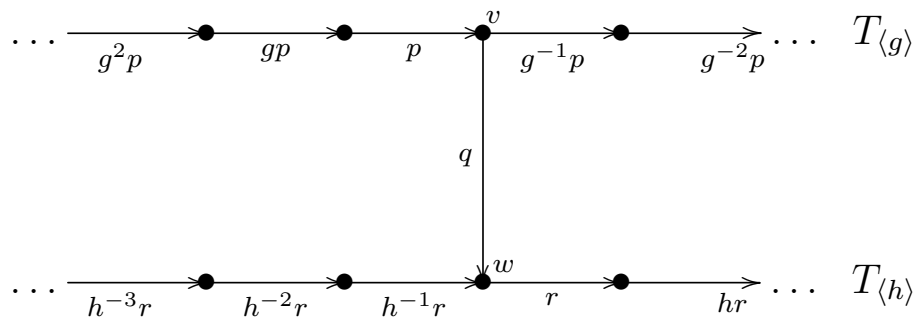

Agora, considerando apenas uma parte do caminho biinfinito de $\mathrm{g}\left(\ldots \cdot g^{2} p \cdot g p \cdot p\right)$, diferentemente do caso do teorema anterior, podemos assumir que a maior aresta $e$ de $p$ em relação à ordem $\prec$ satisfaz ge $\prec e$. Do mesmo modo, podemos ter uma maior aresta em $q$ e em $r$. Então, o caminho reduzido biinfinto $\ldots \cdot g^{2} p \cdot g p \cdot p \cdot q \cdot r \cdot h r \cdot h^{2} r \cdot \ldots$ possui uma maior aresta em $p \cdot q \cdot r$. Portanto, $T_{G}$ possui pelo menos uma ponte e $B(G)$ é um conjunto não vazio.

Para o próximo resultado, considere $I$ uma ilha arbitrária do conjunto de ilhas $I(G)$ e seja $G_{I}=\{g \in G \mid g I \subseteq I\}$ o estabilizador dessa ilha.

Teorema 3.51. Se G é finitamente gerado e não trivial, então os estabilizadores das ilhas são não triviais.

Demonstração. Seja $I$ uma ilha arbitrária de $I(G)$ e $v$ um vértice qualquer em $I$. Como $G \neq\{1\}$, existe algum caminho infinito $p$ em $T_{G}$ começando em $v$. Defina

$$
N:=\left|G \backslash E^{T_{G}}\right|+1
$$

e note que, como $G$ é finitamente gerado, o quociente $G \backslash E^{T_{G}}$ é finito, portanto $\left|G \backslash E^{T_{G}}\right|$ é finito e, assim, $N$ é um número finito. 
Sejam $e_{1}, e_{2}, \ldots, e_{s}$ pontes de $T_{G}$ com distância no máximo $N$ do vértice $v$ escolhido, de modo que $e_{1}>e_{2}>\ldots>e_{s}$. Considere o menor $i$ tal que o caminho $p$ atravessa a ponte $e_{i}$.

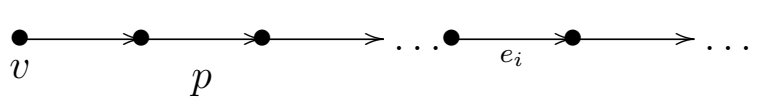

Podemos substituir a calda do caminho infinito $p$ de $e_{i}$ em diante por um caminho em $T_{G}$ formado por arestas de comprimento menor do que $e_{i}$. Desse modo, a aresta $e_{i}$ deixará de ser ponte para esse caminho $p$. Como temos $s$ pontes que distam no máximo $N$ de $v$, depois de fazer $s$ de tais substituições de caldas, obtemos um novo caminho infinito $p$ começando em $v$ que não atravessa nenhuma ponte em uma distância de no máximo $N$ de $v$.

Então, as primeiras $N$ arestas de $p$ estão em $I$ e, assim,

$$
\left|E^{I}\right| \geq N \Longrightarrow\left|E^{I}\right|>N-1=\left|G \backslash E^{T_{G}}\right| .
$$

Logo, existe $e \in E^{T_{G}}$ e $g \in G$ tal que $e \in E^{I}$, ge $\in E^{I}$ e $g e \neq e$, então $g I \cap I \neq \emptyset$. Portanto, existe $g \in G_{I}=\{g \in G \mid g I \subseteq I\}$ e $G_{I} \neq\{1\}$.

Então, como escolhemos uma ilha arbitrária $I$ de $I(G)$, o estabilizador de cada ilha de $I(G)$ será não trivial.

Agora, usando os teoremas anteriores, vamos ver que, para cada $I \in I(G)$, temos $\operatorname{rank}\left(G_{I}\right)=1$. De fato, $G_{I}$ é um fator livre de $G$ e $T_{G_{I}}$ está em $I$. Então, $B\left(G_{I}\right)$ está em $I(G)=T_{G} \backslash B(G)$, mas por outro lado também está em $B(G)$, logo o conjunto de pontes $B\left(G_{I}\right)$ é vazio, ou seja, não existem pontes em $G_{I}$. Pelo Teorema $3.50, \operatorname{rank}\left(G_{I}\right)<2$ e como $G_{I} \neq\{1\}$, segue que $\operatorname{rank}\left(G_{I}\right)=1$.

Provado isso, vamos ver de que maneira o grafo quociente $G \backslash \mathcal{T}_{G}$ está relacionado com $\bar{r}(G)$, pois $G$ pode não agir livremente em $\mathcal{T}_{G}$.

A ideia é ver o grupo $G$ como grupo fundamental de um grafo de grupos específicos, como definimos na Seção 2.5. Nesse caso, vamos considerar $G$ como o grupo fundamental do grafo de grupos $\left(\mathbb{G}, G \backslash \mathcal{T}_{G}\right)$ associado ao grafo quociente $G \backslash \mathcal{T}_{G}$. Então, consideraremos o grupo de vértices $G_{i}$, para $i \in V^{\mathcal{T}_{G}}$, como sendo o estabilizador de uma ilha de $\mathcal{T}_{G}$, e o grupo de arestas $G_{e}$, onde $e \in E^{\mathcal{T}_{G}}$, como sendo o estabilizador de uma ponte de $\mathcal{T}_{G}$.

Vamos calcular agora a característica de Euler do grupo G. Para isso, temos uma definição generalizada de característica de Euler, a qual foi definida por Serre em [24]. 
Definição 3.52. Seja $G$ o grupo fundamental de um grafo de grupos $(\mathbb{G}, Y)$, composto pelo grupo de vértices $G_{v}$ com $v \in V^{Y}$ e pelo grupo de arestas $G_{e}$, onde $e \in E^{Y}$, então a característica de Euler $\chi(G)$ de $G$ é definida como:

$$
\chi(G)=\sum_{i \in V^{Y}} \chi\left(G_{i}\right)-\sum_{e \in E^{Y}} \chi\left(G_{e}\right) .
$$

Assim, no caso em que o grupo $G$ é grupo fundamental do grafo de grupos $\left(\mathbb{G}, G \backslash \mathcal{T}_{G}\right)$, temos que $G_{e}=\{1\}$, pois a ação de $G$ no conjunto $E^{\mathcal{T}_{G}}=B(G)$ é livre, e então o conjunto $B(G)$ possui estabilizador trivial. Logo, teremos $\chi\left(G_{e}\right)=\chi(\{1\})=1$ e a característica de Euler de $G$ será:

$$
\begin{aligned}
\chi(G) & =\sum_{i \in G \backslash I(G)} \chi\left(G_{i}\right)-\sum_{e \in G \backslash B(G)} \chi\left(G_{e}\right) \\
& =\sum_{i \in G \backslash I(G)} \chi\left(G_{i}\right)-\sum_{e \in G \backslash B(G)} 1 \\
& =\sum_{i \in G \backslash I(G)} \chi\left(G_{i}\right)-|G \backslash B(G)| .
\end{aligned}
$$

Agora, como $\bar{r}(G)=\max \{0,-\chi(G)\}$, se $G$ é não trivial temos $\bar{r}(G)=-\chi(G)$. Portanto, se $\sum_{i \in G \backslash I(G)} \chi\left(G_{i}\right)=0$ seguirá o resultado (3.8) que queremos: $\bar{r}(G)=|G \backslash B(G)|$.

Note que, pelo Teorema 3.51, se $G$ é finitamente gerado, as ilhas possuem estabilizadores não triviais e concluímos que $\operatorname{rank}\left(G_{I}\right)=1$. Então, $\chi\left(G_{i}\right)=1-\operatorname{rank}\left(G_{i}\right)=1-1=0$, para cada $i \in G \backslash I(G)$. Logo, segue que

$$
\bar{r}(G)=|G \backslash B(G)|
$$

Observação 3.53. Como observado anteriormente, se $G$ é o grupo trivial já temos que $\bar{r}(G)=|G \backslash B(G)|$. Agora, se $G$ não é finitamente gerado, teremos:

$$
\bar{r}(G)=-\chi(G)=|G \backslash B(G)|-\sum_{i \in G \backslash I(G)} \chi\left(G_{i}\right) \leq|G \backslash B(G)| .
$$

Então, se $\bar{r}(G)=\infty$ seguirá que $|G \backslash B(G)|=\infty$, e o resultado (3.8) valerá da mesma maneira.

Portanto, o Teorema SHNC 3.43 está demonstrado. 


\subsection{O limitante superior é sharp}

Agora, provada a conjectura, dados dois subgrupos finitamente gerados $A$ e $B$ do grupo livre $\Gamma$, de postos $m$ e $n$, respectivamente, se $N$ é o posto da interseção desses dois subgrupos, sabemos que $N-1 \leq(m-1)(n-1)$. Pensando nisso, uma questão interessante é questionar-se se esse limitante é o melhor possível. Nesse sentido, no ano de 2005, Richard Kent mostrou em [14] que todos os números entre 0 e $(m-1)(n-1)+1$ podem ocorrer como posto da interseção de $A$ e $B$ e, assim, o limite superior sugerido pela conjectura não pode ser melhorado. Nesse caso, dizemos que o limitante superior é sharp.

Para essa demonstração, precisamos provar o teorema abaixo e, na sequência, um corolário do mesmo nos garantirá que o limite superior é o melhor possível.

Teorema 3.54. Seja $\Gamma=\Gamma(a, b)$ um grupo livre de posto dois. Sejam $H_{k, l}^{m}=\left\langle a, b a b^{-1}, \ldots, b^{k} a b^{-k}, b^{k+1} a^{n-l} b^{-(k+1)}, b^{k+2} a^{n} b^{-(k+2)}, \ldots, b^{m-1} a^{n} b^{-(m-1)}\right\rangle e$ $K=\left\langle b, a b a^{-1}, \ldots, a^{n-1} b a^{-(n-1)}\right\rangle$ subgrupos de $\Gamma$, onde $0 \leq k \leq m-2$ e $0 \leq l \leq n-1$. $\operatorname{Então} \operatorname{rank}\left(H_{k, l}^{m} \cap K\right)=k(n-1)+l$.

Demonstração. Seja $X$ um buquê de dois círculos tal que $\pi_{1}(X)=\Gamma$. Seja $H$ um subgrupo finitamente gerado de $\Gamma$, logo existe um recobrimento $X(H)$ de $H$ tal que $\pi_{1}(X(H))=H$. Então, dados os dois subgrupos $H, K \leq \Gamma$ temos os grafos finitos associados a cada um deles e, assim, podemos construir o grafo de sua interseção (veja Exemplos 2.14 e 2.15). Analisando esse grafo, podemos obter o valor do posto da interseção desses subgrupos, como visto na Observação 2.41, e, assim, chegar no resultado desejado.

Para desenhar os grafos dos subgrupos e da interseção, inicialmente vamos fixar o valor $k=m-2$ e variar o valor de $l$, sendo que, para ser possível fazer o desenho do grafo, daremos valores para $m$ e $n$.

- Caso $k=m-2, l=n-1$. $H_{m-2, n-1}^{m}=\left\langle a, b a b^{-1}, \ldots, b^{k} a b^{-k}, b^{k+1} a^{n-l} b^{-(k+1)}\right\rangle$ e $K=\left\langle b, a b a^{-1}, \ldots, a^{n-1} b a^{-(n-1)}\right\rangle$

Tomando $m=4$ e $n=4$, temos $k=2$ e $n-l=1$, assim os subgrupos obtidos serão: $H_{2,3}^{4}=\left\langle a, b a b^{-1}, b^{2} a b^{-2}, b^{3} a b^{-3}\right\rangle$ e $K=\left\langle b, a b a^{-1}, a^{2} b a^{-2}, a^{3} b a^{-3}\right\rangle$.

Para desenhar os grafos dos subgrupos acima, utiliza-se a ideia descrita após a Definição 2.13, ou seja, considera-se um vértice base e os caminhos que saem e 
retornam para esse vértice são os geradores do subgrupo. As arestas serão marcadas pelos geradores do grupo livre $\Gamma$, isto é, por $a$ e $b$ e, para facilitar o entendimeto, vamos considerar sempre que as arestas com um pequeno triângulo $(\triangleright)$ no centro representarão as arestas marcadas por $a$ e as arestas sem o triângulo as marcadas por $b$. Além disso, o vértice base será sempre o primeiro vértice do lado esquerdo do grafo. Desse modo, os respectivos grafos serão:

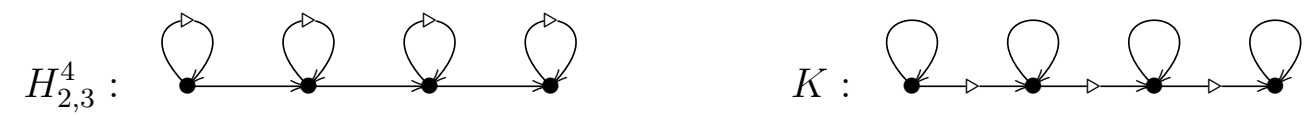

Agora, analisando os dois grafos acima e seguindo a ideia da construção descrita no Exemplo 2.15, obtemos o grafo da interseção. Esse grafo será marcado pelos elementos da base de $\Gamma$ e seus vértices pertencerão ao conjunto $V^{H} \times V^{K}$. Assim, formamos os possíveis pares ordenados com os vértices dos grafos acima e em seguida, para descobrir quando eles serão conectados por uma aresta marcada por $a$ ou por $b$, analisamos coordenada a coordenada dos pares, vendo se são ou não conectados nos grafos $H$ e $K$. Seguindo essa ideia, o grafo da interseção dos subgrupos $H_{2,3}^{4}$ e $K$ acima, será:

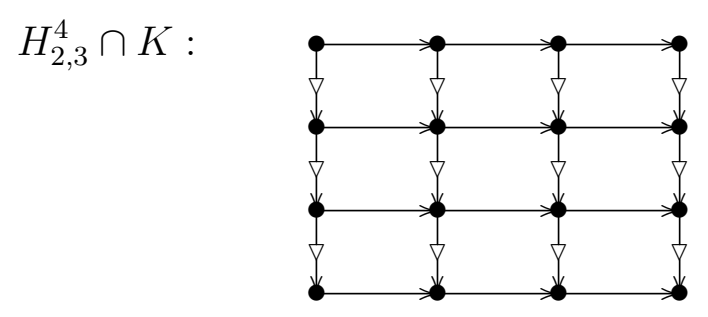

Fazendo a contagem do número de vértices e arestas do grafo acima, pela Observação 2.41, obtemos:

$$
\operatorname{rank}\left(H_{2,3}^{4} \cap K\right)=E^{H_{2,3}^{4} \cap K}-V^{H_{2,3}^{4} \cap K}+1=9=(m-1)(n-1)=k(n-1)+l .
$$

- Caso $k=m-2, l=n-2$.

$H_{m-2, n-2}^{m}=\left\langle a, b a b^{-1}, \ldots, b^{k} a b^{-k}, b^{k+1} a^{n-l} b^{-(k+1)}\right\rangle$ e $K=\left\langle b, a b a^{-1}, \ldots, a^{n-1} b a^{-(n-1)}\right\rangle$.

Tomando $m=4$ e $n=4$, temos $k=2$ e $n-l=2$. Seguindo a mesma ideia da construção anterior, o grafo $H$ será modificado e grafo de $K$ permanecerá o mesmo:

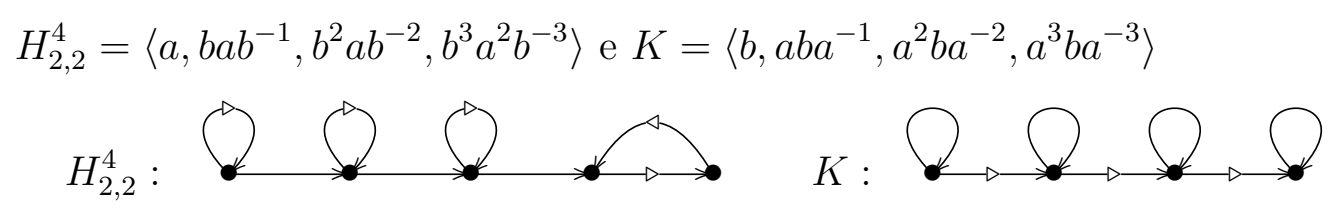


O grafo da interseção será diferente do anterior, teremos arestas diagonais entrelaçadas no lado direito do grafo, onde ambas representam arestas marcadas por $a:$

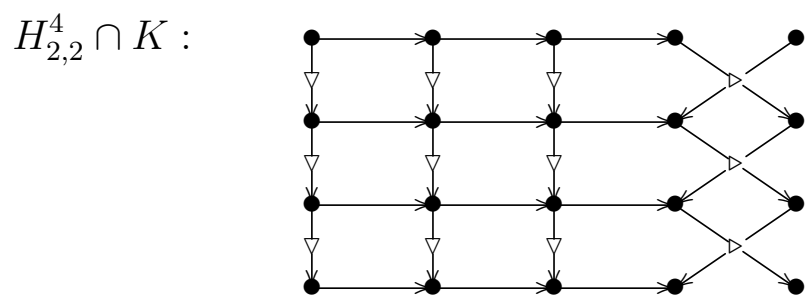

Analisando novamente o grafo, temos:

$$
\operatorname{rank}\left(H_{2,2}^{4} \cap K\right)=8=(m-1)(n-1)-1=k(n-1)+l .
$$

Considerando o caso em que $k=m-2$ e $l=n-3$ e construindo os grafos de maneira análoga à apresentada acima, obtemos que

$$
\operatorname{rank}\left(H_{m-2, n-3}^{m} \cap K\right)=(m-1)(n-1)-2=k(n-1)+l .
$$

Seguindo com essa construção, para o caso $l=n-j$, onde $1 \leq j \leq n$, teremos:

$$
\operatorname{rank}\left(H_{m-2, n-j}^{m} \cap K\right)=(m-1)(n-1)-(j-1)
$$

Note que o posto acima é igual a $k(n-1)+l$ quando $k=m-2$ e $l=n-j$.

Faremos agora o caso em que $l=0$ e construiremos o grafo associado a $H_{m-2,0}^{m} \cap K$. Para isso, consideraremos novamente que $m=4$ e $n=4$, então $k=2$ e $n-l=4$, e teremos os subgrupos $H_{2,0}^{4}=\left\langle a, b a b^{-1}, b^{2} a b^{-2}, b^{3} a^{4} b^{-3}\right\rangle$ e $K=\left\langle b, a b a^{-1}, a^{2} b a^{-2}, a^{3} b a^{-3}\right\rangle$. Construindo os grafos da mesma maneira que as anteriores, obtemos:

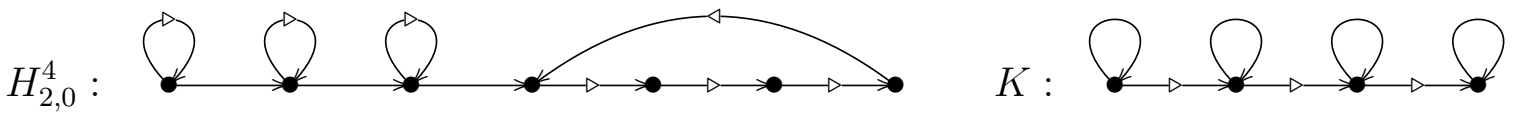

O grafo da interseção terá novamente arestas diagonais, todas marcadas por $a$ :

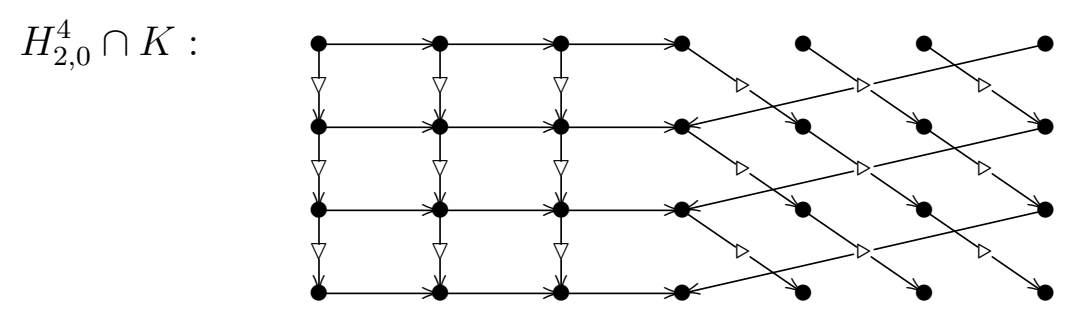


Note que nesse caso o posto da interseção será $\operatorname{rank}\left(H_{2,0}^{4} \cap K\right)=6=(m-1)(n-1)-3$, satisfazendo a igualdade (3.9).

Agora, desenharemos o grafo da interseção $H_{m-3, n-1}^{m-1} \cap K$ e veremos que ele possui o mesmo posto do grafo da interseção acima, do caso $l=0$. Para isso, se tomarmos os valores $m=4$ e $n=4$, teremos os sugrupos $H_{1,3}^{3}=\left\langle a, b a b^{-1}, b^{2} a b^{-2}\right\rangle$ e $K=\left\langle b, a b a^{-1}, a^{2} b a^{-2}, a^{3} b a^{-3}\right\rangle$. Então:

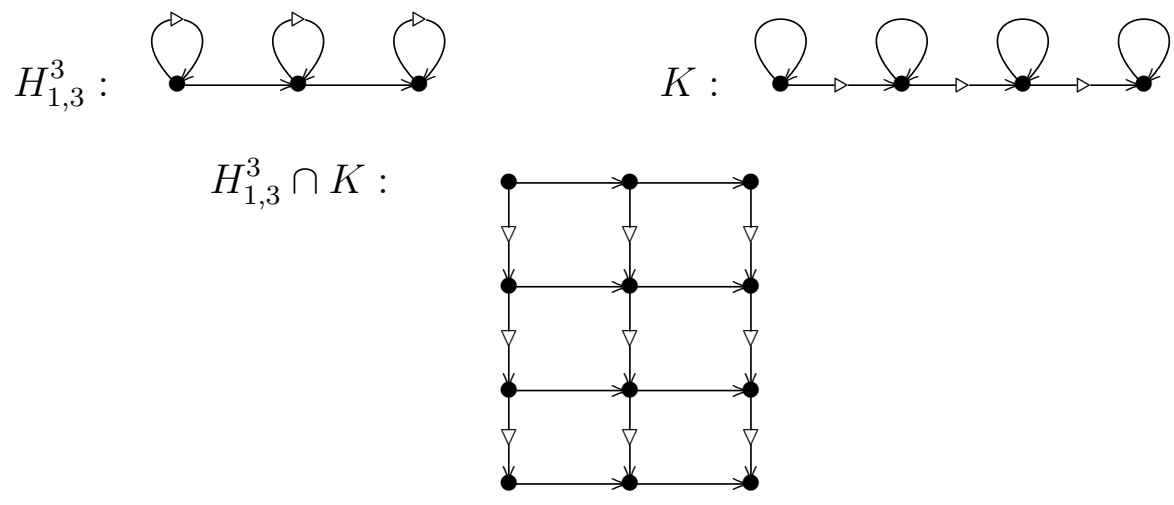

Note que esse grafo difere do grafo $H_{2,0}^{4} \cap K$ apenas por árvores anexadas nos vértices do lado direito, e que o posto da interseção será o mesmo, já que as árvores anexadas deixam o posto intacto. De fato, temos $\operatorname{rank}\left(H_{1,3}^{3} \cap K\right)=6=\operatorname{rank}\left(H_{2,0}^{4} \cap K\right)$. Assim, de modo geral teremos que $\operatorname{rank}\left(H_{m-2,0}^{m} \cap K\right)=\operatorname{rank}\left(H_{m-3, n-1}^{m-1} \cap K\right)$.

De maneira análoga, podemos relacionar outros grafos da interseção desses subgrupos $H_{k, l}^{m}$ e $K$. Por exemplo, o grafo associado a $H_{m-3, n-2}^{m} \cap K$ difere do grafo $H_{m-3, n-2}^{m-1} \cap K$ apenas por árvores anexadas em alguns de seus vértices, como segue abaixo.

- $H_{m-3, n-2}^{m}=\left\langle a, b a b^{-1}, \ldots, b^{k+1} a^{n-l} b^{-(k+1)}, b^{k+2} a^{n} b^{-(k+2)}\right\rangle$

$K=\left\langle b, a b a^{-1}, \ldots, a^{n-1} b a^{-(n-1)}\right\rangle$

Para o caso específico de $m=4$ e $n=4$, temos:

$$
\begin{aligned}
& H_{1,2}^{4}=\left\langle a, b a b^{-1}, b^{2} a^{2} b^{-2}, b^{3} a^{4} b^{-3}\right\rangle \\
& K=\left\langle b, a b a^{-1}, a^{2} b a^{-2}, a^{3} b a^{-3}\right\rangle
\end{aligned}
$$

$H_{1,2}^{4}$ :

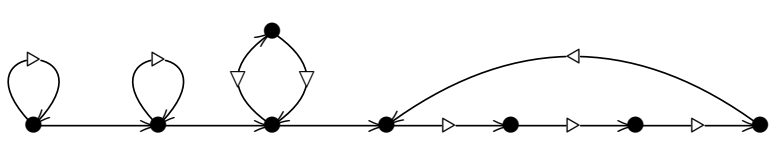

$K$ :

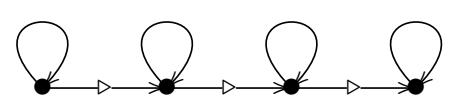




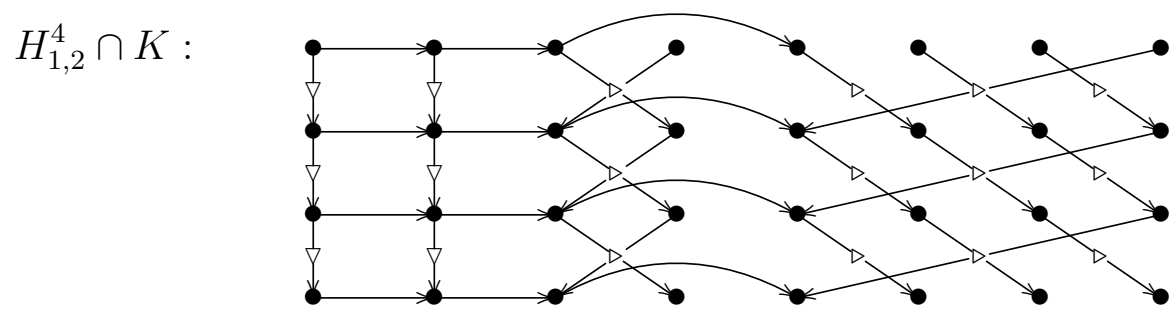

- $H_{m-3, n-2}^{m-1}=\left\langle a, b a b^{-1}, \ldots, b^{k+1} a^{n-l} b^{-(k+1)}\right\rangle$

$K=\left\langle b, a b a^{-1}, \ldots, a^{n-1} b a^{-(n-1)}\right\rangle$

E para o caso específico de $m=4$ e $n=4$, temos:

$$
\begin{aligned}
& H_{1,2}^{3}=\left\langle a, b a b^{-1}, b^{2} a^{2} b^{-2}\right\rangle \\
& K=\left\langle b, a b a^{-1}, a^{2} b a^{-2}, a^{3} b a^{-3}\right\rangle
\end{aligned}
$$
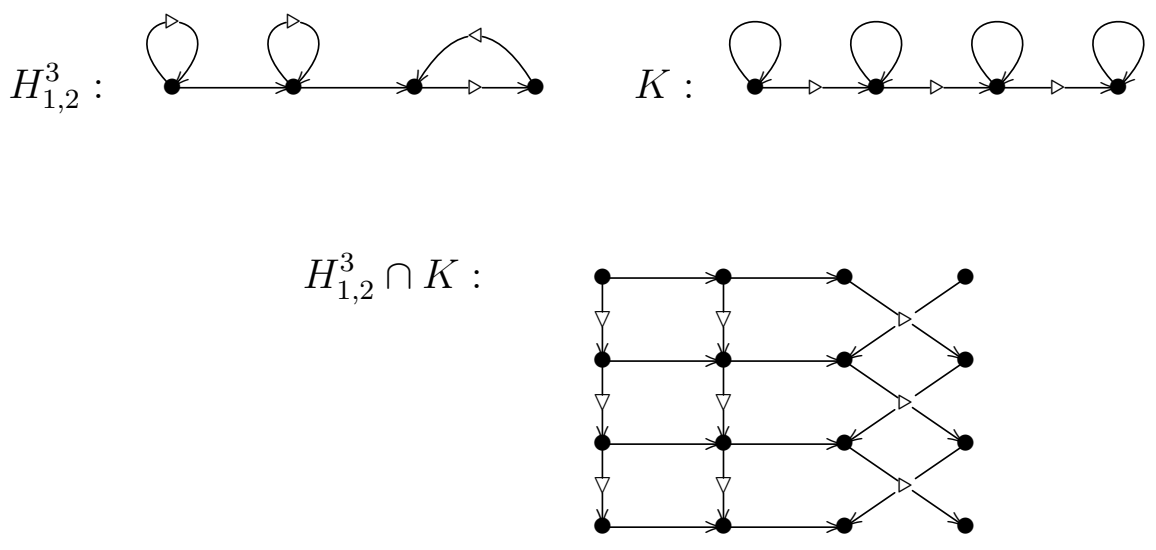

Note que ambos os grafos da interseção $H_{1,2}^{4} \cap K$ e $H_{1,2}^{3} \cap K$ possuem o mesmo posto:

$$
\operatorname{rank}\left(H_{1,2}^{4} \cap K\right)=k(n-1)+l=5=\operatorname{rank}\left(H_{1,2}^{3} \cap K\right) .
$$

Como observado, o posto da interseção desses grupos não se altera com o anexo de árvores em seus vértices. Então, podemos concluir a demonstração por indução em $m$, da maneira como descreveremos agora.

Fixemos os valores de $n, l$ e $k$, sendo que esse último dependerá do valor de $m$. Observando o que fizemos anteriormente, para o caso $m=4$ temos válido $\operatorname{rank}\left(H_{k, l}^{m} \cap K\right)=$ $k(n-1)+l$. Suponhamos que o resultado vale para o caso $m-1$.

Note que, comparando os grafos $H_{m-2,0}^{m} \cap K$ e $H_{m-3, n-1}^{m-1}, \quad H_{m-3, n-2}^{m} \cap K$ e $H_{m-3, n-2}^{m-1} \cap K$, que diferem por árvores anexadas em alguns de seus vértices, obtemos o mesmo posto da 
interseção em cada caso. Então, podemos comparar todo grafo da interseção em que $H$ tem índice $m$ com um grafo da interseção de mesmo posto em que $H$ tem índice $m-1$, e, assim, o resultado segue por indução. Portanto, $\operatorname{rank}\left(H_{k, l}^{m} \cap K\right)=k(n-1)+l$, para $0 \leq k \leq m-2$ e $0 \leq l \leq n-1$.

Agora, o corolário abaixo nos fornece os subgrupos que procuramos para provar que o limite de Hanna Neumann não pode ser melhorado.

Corolário 3.55. Seja $F$ um grupo livre e sejam $m, n \geq 2$ números naturais. Seja $N$ o número natural tal que $1 \leq N-1 \leq(m-1)(n-1)$. Então, existem subgrupos $H$ e $K$ de $\Gamma$, de postos $m$ e $n$ respectivamente, tais que o posto de $H \cap K$ é $N$.

Demonstração. Note que o Teorema 3.54 anterior produz os subgrupos desejados para todo $N$ com $N-1 \leq(m-1)(n-1)-1$. Para alcançarmos o limite superior, ou seja, $N-1=(m-1)(n-1)$, basta considerar $H=\left\langle a, b a b^{-1}, \ldots, b^{m-2} a b^{-(m-2)}, b^{m-1}\right\rangle=H_{m-2, n}^{m}$ e $K=\left\langle b, a b a^{-1}, \ldots, a^{n-2} b a^{n-2}, a^{n-1}\right\rangle$. De fato, teremos $\operatorname{rank}\left(H_{m-2, n}^{m} \cap K\right)=N$ :

$$
\begin{aligned}
\operatorname{rank}\left(H_{m-2, n}^{m} \cap K\right) & =k(n-1)+l \\
& =(m-2)(n-1)+n \\
& =(m-1)(n-1)+1 .
\end{aligned}
$$

Agora, vejamos que os grupos considerados são os que procurávamos atráves de um exemplo. Tomando os valores $m=4$ e $n=4$ teremos $k=2$ e $n-l=0$ e os subgrupos serão: $H=\left\langle a, b a b^{-1}, b^{2} a b^{-2}, b^{3}\right\rangle$ e $K=\left\langle b, a b a^{-1}, a^{2} b a^{-2}, a^{3}\right\rangle$. Faremos a construção de seus respectivos grafos e do grafo interseção:

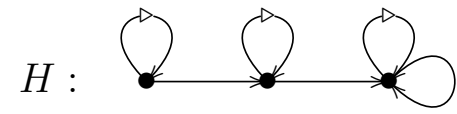

$K$ :
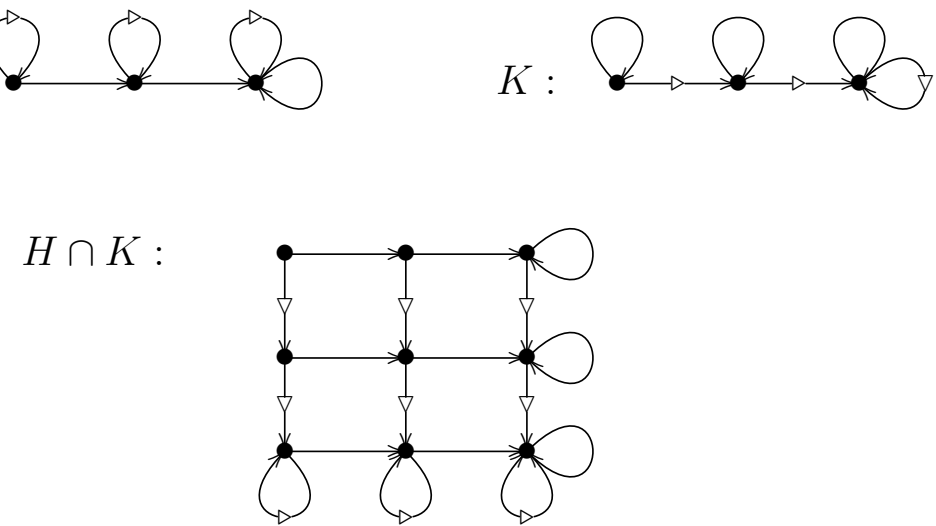

Note então que $\operatorname{rank}(H \cap K)=18-9+1=10=(m-1)(n-1)+1$, como queríamos. 


\section{Considerações Finais}

A Conjectura de Hanna Neumann era um problema em aberto desde a década de 1950 e através dos trabalhos de diversos matemáticos ao longo dos anos subsequentes, foi provada ser verdadeira recentemente. Como vimos, primeiramente Howson mostrou que a interseção de subgrupos finitamente gerados de um grupo livre é finitamente gerada. Em 1957, Hanna Neumann apresentou um limitante para o posto dessa interseção e conjecturou um limite melhor possível. Somente no ano de 2011, Mineyev e Friedman provaram a validade da conjectura, em trabalhos independentes, e esse resultado nos fornece uma ferramenta muito útil para o cálculo do posto da interseção de dois subgrupos finitamente gerados de um grupo livre.

Uma questão natural, seria se existe um resultado análogo para a interseção de subgrupos finitamente gerados de um produto livre de grupos. Em 1966, B. Baumslag em seu artigo Intersections of finitely generated subgroups in free products, estabeleceu um resultado análogo ao provado por Howson para um produto livre $A * B$ de grupos $A$ e $B$, mostrando que se $A$ e $B$ possuem a propriedade de Howson, então $A * B$ também possui. Em 1990, T. Soma em seu artigo Intersection of finitely generated surface groups, foi o primeiro a obter um limitante explícito para o posto da interseção nesse caso. Na sequência, a partir do Teorema do Subgrupo de Kurosh e da definição do posto de Kurosh, que verificou-se ser o posto apropriado a ser utilizado nesse caso de subgrupos, alguns matemáticos buscaram provar a desigualdade de Hanna Neumann para o caso da interseção de subgrupos de um produto livre de grupos $G=*_{i \in I} A_{i}$. Em 2014, Y. Antolín, A. Martino e I. Schwabrow, provaram que o posto reduzido de Kurosh da interseção de dois subgrupos de um produto livre de grupos, ordenados à direita, é limitado superiormente pelo produto dos postos reduzidos de Kurosh de cada um deles. Esse resultado, assim como a definição do posto de Kurosh, pode ser encontrado no artigo intitulado 
Kurosh rank of intersections of subgroups of free products of right-orderable groups e, em particular, é um análogo a versão forte da Conjectura de Hanna Neumann para grupos livres. 


\section{Referências Bibliográficas}

[1] BAUMSLAG, G., Topics in Combinatorial Group Theory. Birkhauser, 1993.

[2] BOGOPOLSKI, O., Introduction to Group Theory. European Mathematical Society, 2008.

[3] BURNS, R. G., On the intersection of finitely generated subgroups of a free group. Mathematische Zeitschrift, vol. 119, 1971, p. 121-130.

[4] COTE, L., Free groups and graphs: the Hanna Neumann Theorem. Disponível em: http://www.math.uchicago.edu/ may/VIGRE/VIGRE2008/REUPapers/Cote.pdf. Acesso em 27 set. 2015.

[5] DICKS, W., Equivalence of the strengthened Hanna Neumann conjecture and the amalgamated graph conjecture. Inventiones Mathematicae, vol. 117, 1994, n. 3, p. 373-389.

[6] DICKS, W., Groups, Trees and Projective Modules (Lecture Notes in Mathematics, vol. 790). Springer-Verlag Berlin, 1980.

[7] DICKS, W., FORMANEK, E., The rank three case of the Hanna Neumann conjecture. Journal of Group Theory, vol. 4, 2001, n. 2, p. 113-151.

[8] DICKS, W., Simplified Mineyev. Disponível em: http://mat.uab.es/ dicks/ SimplifiedMineyev.pdf. Acesso em 14 nov. 2015.

[9] DICKS, W., Two corrections. Disponível em: http://mat.uab.es/ dicks/two\%20corrections.pdf. Acesso em 25 jan. 2016. 
[10] FRIEDMAN, J., Sheaves on Graphs, Their Homological Invariants, and a Proof of the Hanna Neumann Conjecture. American Mathematical Society, 2014.

[11] HOWSON, A. G., On the intersection of finitely generated free groups. Journal of the London Mathematical Society, vol. 29, 1954, p.428-434.

[12] JOHnSON, D. L., Presentation of groups. Cambridge University Press, 2 ed., 1997.

[13] KHAN, B., Positively generated subgroups of free groups and the Hanna Neumann conjecture. Contemporary Mathematics, American Mathematical Society, vol. 296, 2002, p. $155-170$.

[14] KENT, R. P., Achievable ranks of intersections of finitely generated free groups. International Journal of Algebra and Computation, Vol. 15, n. 2, 2005, p.339-341.

[15] MAC LANE, S., Categories for the Working Mathematician. Springer-Verlag New York, 1971.

[16] MEAKIN, J., WEIL, P., Subgroups of free groups: a contribution to the Hanna Neumann conjecture. Proceedings of the Conference on Geometric and Combinatorial Group Theory, Part I (Haifa, 2000). Geometriae Dedicata, vol. 94, 2002, p. 33-43.

[17] MINEYEV, I., Submultiplicativity and the Hanna Neumann Conjecture. Ann. of Math., 175, 2012, p. 393-414.

[18] MINEYEV, I., Groups, graphs, and the Hanna Neumann Conjecture. Jornal of Topology and Analysis (JTA), vol. 4, n. 1, 2012, p. 1-12.

[19] MINEYEV, I., The topology and analysis of the Hanna Neumann Conjecture. Journal of Topology and Analysis (JTA), vol. 3, 2011, p. 307-376.

[20] NEUMANN, H., On the intersection of finitely generated free groups. Publicationes Mathematicae Debrecen, vol. 4, 1956, p.186-189.

[21] NEUMANN, H., On the intersection of finitely generated free groups. Addendum. Publicationes Mathematicae Debrecen, vol. 5, 1957, p. 128. 
[22] NEUMANN, W.D., On the intersection of finitely generated subgroups of free groups. Groups-Canberra 1989, p. 161-170. Lecture Notes in Mathematics, vol. 1456, Springer, Berlin, 1990.

[23] REYES, V., Group Theory. Disponível em: http://math.hunter.cuny.edu/olgak/Book \%20group\%20theory.pdf. Acesso em: 14 nov. 2015.

[24] SERRE, J-P., Trees. Springer-Verlag, 1980.

[25] STALLINGS, J. R., Topology of finite graphs. Inventiones Mathematicae, vol. 71, 1983, p. 551-565.

[26] STILLWELL, J., Classical Topology and Combinatorial Group Theory. SpringerVerlag, 2 ed., 1993.

[27] TARDOS, G., On the intersection of subgroups of a free group. Inventiones Mathematicae, vol. 108, 1992, n. 1, p. 29-36.

[28] TARDOS, G., Towards the Hanna Neumann conjecture using Dicks method. Inventiones Mathematicae, vol. 123, 1996, p. 95-104. 